\title{
CLASS B MONITORING PROGRAM HANDBOOK FOR SOUTHEASTERN SITES WITH DATA BASED \\ ON INIT2 SOFTWARE
}

\section{(Applicable to Heating Season)}

\author{
David H. Gustashaw \\ Energy Systems Group \\ ESG, Inc. \\ Atlanta, GA 30341
}

$$
\text { July } 1983
$$

\section{NOTICE}

PORTIONS OF THIS REPORT ARE ILLEGIBLE.'

It has been reproduced from the best available copy to permit the broadest possible availability.

MN ONLY

Prepared for

U. S. DEPARTMENT OF Energy

Under Contract Number DE-ACO2-82CHI 0122 


\section{DISCLAIMER}

This report was prepared as an account of work sponsored by an agency of the United States Government. Neither the United States Government nor any agency Thereof, nor any of their employees, makes any warranty, express or implied, or assumes any legal liability or responsibility for the accuracy, completeness, or usefulness of any information, apparatus, product, or process disclosed, or represents that its use would not infringe privately owned rights. Reference herein to any specific commercial product, process, or service by trade name, trademark, manufacturer, or otherwise does not necessarily constitute or imply its endorsement, recommendation, or favoring by the United States Government or any agency thereof. The views and opinions of authors expressed herein do not necessarily state or reflect those of the United States Government or any agency thereof. 


\section{DISCLAIMER}

Portions of this document may be illegible in electronic image products. Images are produced from the best available original document. 


\section{NOTICE}

This report was prepared as an account of work sponsored by the United States Government. Neither the United States nor the Deplartment of Energy, nor any of their employees. nor any of their contractors, subcontractors, or their employees, makes any warranty, express or implied, or assumes any legal liability or responsibility for the accuracy, completeness, or usefulness of any information, apparatus, product or process disclosed, or represents that its use would not infringe privately-owned rights. 


\section{FOREWORD}

This report, as prepared by ESG; Inc., is based, in part, upon an earlier technical effort conducted for the U.S. Department of Energy by the Southern Solar Energy Center as one of the regional solar energy centers. Key personnel worked on the project since its inception so that continuity was assured. The cooperation of personnel in the Chicago Operations Office and the Office of Conservation and Renewable Energy of the U.S. Department of Energy during this period was very important and is gratefully acknowledged. 
TABLE OF CONTENTS

Page

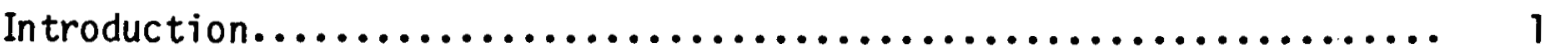

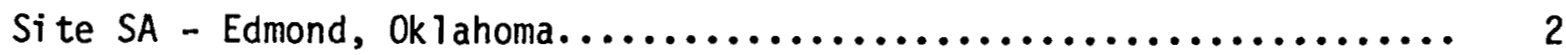

Site SB - Houston, Texas............................ 12

Site SF - Suwanee, Georgia............................ 23

Site SG - Atlanta, Georgia........................... 35

Si te SJ - Manning, South Carolina........................ 48

Site SK - Black Mountain, North Carolina..................... 60

Si te $S L$ - Stephens City, Virginia....................... 70

Site SN - Richmond, Virginia........................ 82

Appendix A - Site Photograph Tabulation.................... 95 
CLASS B MONITORING PROGRAM HANDBOOK FOR SOUTHEASTERN SITES WITH DATA BASED ON INIT2 SOFTWARE

Introduction

This document provides reference information on eight residences located in the southern part of the U.S. which are being monitored under the Class B Passive Solar Monitoring Program for the U.S. Department of Energy. ESG, Inc. has been responsible for the field instrumentation and data collection on these sites. Overall program coordination is through the Chicago Operations Office of DOE and the Solar Energy Research Institute.

This handbook provides reference information on the sites and basic test conditions for the 1982-83 winter heating season using software which is identified as "INIT2." The manual is intended for use in data analysis work but is limited to this period of time because of basic differences in the control software and the related instrumentation system.

Discussion

This document is separated into individual sections for each site. The following information is provided for each site:

a. Site summary providing information on the structure, the passive solar features, the auxiliary heating system, and other related data.

b. Floor layouts with the sensor locations, etc.

c. The "One Time Value Log" which records the results of field tests and calculations to determine the thermal characteristics, air infiltration rate, and other reference data.

d. The "Analog/Digital Channel Log" which provides calibration data.

e. The "Status Channel Log" which defines various event sensors.

f. Program listings as used during the specified time periods during the heating season.

Appendix A provides a summary of photographs which have been provided separately as part of the site documentation. In general, the photographs showed the individual sensor installations and elevations of each house. They can be used to provide further insight into the passive features and the installation of the data acquisition equipment.

It should be noted that the data collection activity proceeded in a very effective manner during the winter heating season. Downtime was at a minimum. This is the result of continuous attention to field operation needs and prompt action to correct problems, including those associated with interference and lighting transients. Also, good communications with the participating homeowners helped insure that they could provide assistance throughout the test period. 
SITE SA

Mr. \& Mrs. Jim McCaffrey 1301 Timberlake Avenue

Edmond, OK 73034

INIT2 Software Installed: 1/13/83 


\section{SITE SOMHART SREET}

Site Identsficat:on Nurber SA1

Inst. Contr. Name ESG, Inc.

Address 2231 Perimeter Park

Suite 11 At lanta. GA 30341

\section{Locasion:}

Town Edmond, OK

Latilude 3502

Longi=ude 9704

Elevation $1280 \mathrm{FT}$

Nearast Neather Station:

OK l ahoma City, OK

One story residence

Wood and stone veneer $W /$ wood frame Slab on arade

\footnotetext{
Passive/Hybrid 3ystea(s):

Dowinant Heating System Type

Dominant Heatiaz System Area

Other Syszed Tipe

Ocher Syscea Area

Night Insulation
}

duxiliary Heating System:

Syste] and Distrijution Type

Day Theraostat jetsing

Nighc Therzostas Serting

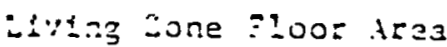

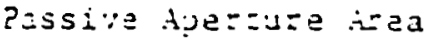

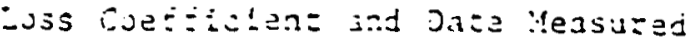

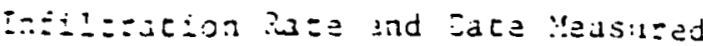

Direct gain

440 FT2 $^{2}$

Venting through slah from clerestery $N / A$

1" polystyrene

Resistance forced air 680

$68^{\circ}$

$2500 E T^{2}$

440 ET2

$L=509$ BTU/HR OF $1 / 12$ and $1 / 13 / 83$

Ne nressure $1 / 11 / 83$ 


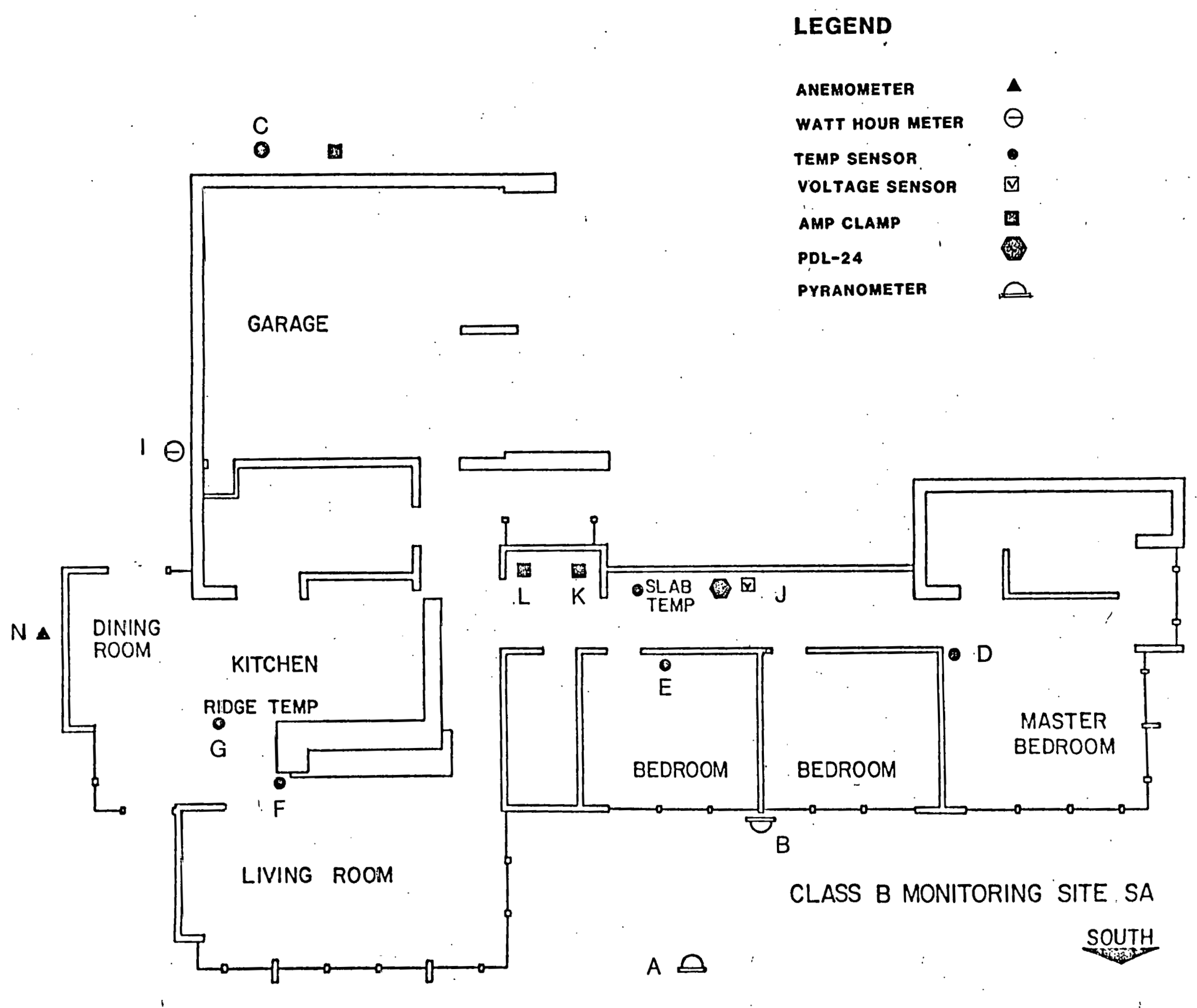




\section{One-Türne Varluge Log}

Variable
Namein
Functions

OT(1)

Overall heat loss

coefficient

$\overrightarrow{O T}(2)$

Furnace delivery

efficiency

OT(3)

fir infiltration rate

OT(4)

Furnace burner

fuel flaw rate

OT(5) Heating vaiue of furnace fuel

OT (6)

Water heater burner

OT(7)

fuelflow rate

Heating value of

water heater fuel

Water lieating jacket heating factor

Water heating space heater factor

Aux. heating set

point temperature

Aux. cooling set

OT (12)

point temperature

OT (13)

Net passive collector area

correction factor

OT(14) Elec. aux. heating voltage multiplier

OT (15) Elec. Water heating voltage multiplier

OT(16) Channel $M$ voltage mult iplier

OT(17) Channel $N$ voltage multiplier

\begin{tabular}{|c|c|c|}
\hline Designation & $\begin{array}{c}\text { Numerical } \\
\text { Value }\end{array}$ & Units \\
\hline$L$ & 509 & $\frac{B T U h}{O F}$ \\
\hline$\overline{n_{f}}$ & 1.0 & -- \\
\hline I & .75 & $\overline{A C H}$ \\
\hline$F_{f}$ & 0 & gal/hr \\
\hline HVF $_{f}$ & 0 & $\overline{B T U / g a}$ \\
\hline$F_{w}$ & 0 & gal/hr \\
\hline$H_{W V F}$ & 0 & BTU/gC \\
\hline$Q_{w}$ & 347 & $\overline{B T U H}$ \\
\hline$N_{w}$ & .2 & -- \\
\hline Tseth & 68 & ${ }^{O} F$ \\
\hline Tsetc & 75 & ${ }^{D_{F}}$ \\
\hline$A_{c}$ & 440 & $\mathrm{ft}^{2}$ \\
\hline$N_{m i}$ & .77 & -- \\
\hline$C_{\text {auxh }}$ & 2 & -- \\
\hline$c_{s}$ & 2 & -- \\
\hline$C_{m}$ & 1 & -- \\
\hline$c_{n}$ & 0 & -- \\
\hline
\end{tabular}

Program 


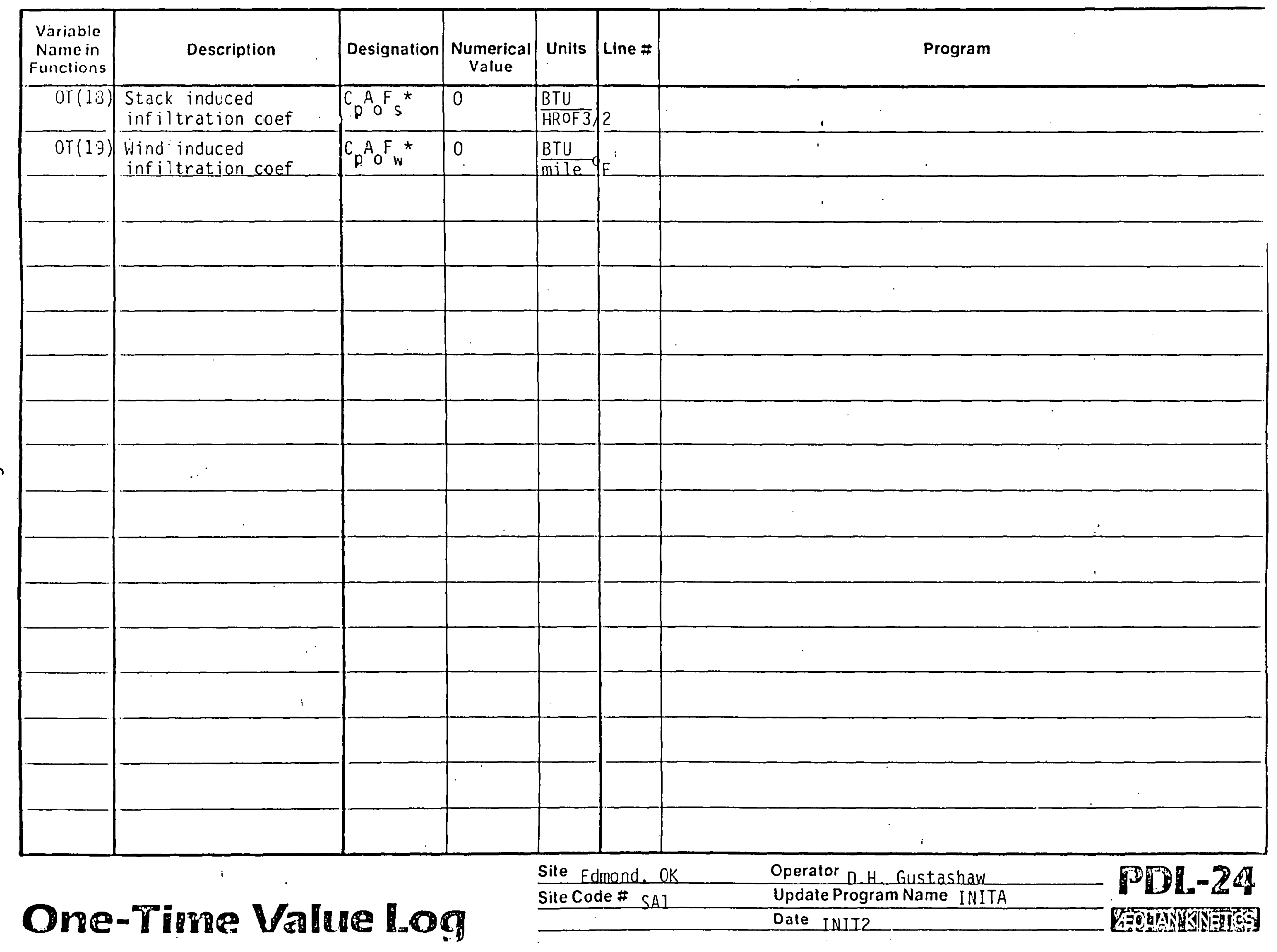




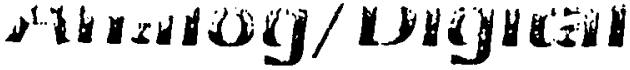 \\ Chamavel Log}

Sensor Terminal $=A 10004$

Sile Edmand, 0 Sile Code \# SAI
Operator D.H. Gustashaw

Updale Program Name INITA Sensor Terminal Coeflicient

\begin{tabular}{|c|c|c|c|c|c|c|c|c|c|c|c|c|c|c|c|}
\hline \multirow{2}{*}{$\begin{array}{l}\bar{a} \\
\frac{c}{c} \\
0 \\
\frac{5}{U}\end{array}$} & \multirow[b]{2}{*}{$\begin{array}{c}\text { Sensor Type } \\
\text { and } \\
\text { Serial }=\end{array}$} & \multirow[b]{2}{*}{$\begin{array}{l}\text { Paranieter } \\
\text { Measured }\end{array}$} & \multirow{2}{*}{ 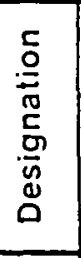 } & \multirow{2}{*}{ 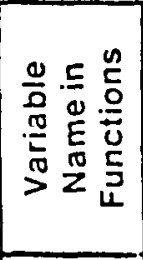 } & \multirow[b]{2}{*}{ 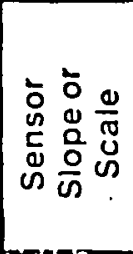 } & \multirow{2}{*}{ 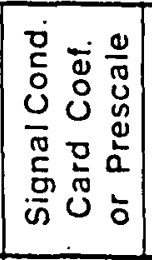 } & \multirow{2}{*}{ 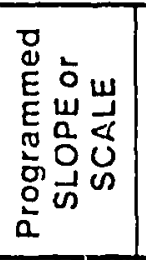 } & \multirow{2}{*}{ 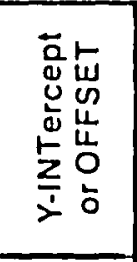 } & \multicolumn{3}{|c|}{$\begin{array}{l}\text { YES }=1 \\
N O=0\end{array}$} & & \multirow[b]{2}{*}{$\stackrel{n}{\stackrel{n}{5}}$} & \multirow{2}{*}{ 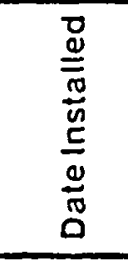 } & \multirow[b]{2}{*}{$\begin{array}{r}\text { Remarks } \\
\ddots \\
\end{array}$} \\
\hline & & & & & & & & & $\begin{array}{l}0 \\
0 \\
0 \\
0 \\
0 \\
0 \\
0 \\
0 \\
\end{array}$ & 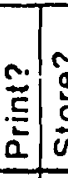 & 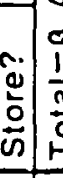 & & & & \\
\hline$A$ & $\begin{array}{l}\text { 1. iCor pyranometer } \\
\text { py } 2784\end{array}$ & $\begin{array}{l}\text { Incident solar } \\
\text { radiation on the } \\
\text { horizontal }\end{array}$ & $I_{h}$ & $F N C(1)$ & $\begin{array}{l}4.72 \\
\times 10^{6}\end{array}$ & $\begin{array}{l}9.935 \\
\times 10^{3}\end{array}$ & 478.38 & 0 & 1 & 1 & 1 & $\overline{0}$ & & $\overline{12 / 81}$ & Center roof at peak \\
\hline$B$ & LiCor pyranometer & $\begin{array}{l}\text { Incident solar } \\
\text { radiation on } \\
\text { collector plane }\end{array}$ & $I_{\text {in }}$ & $F N C(2)$ & $\begin{array}{l}4.15 \\
\times 10^{6}\end{array}$ & $\begin{array}{l}9.963 \\
\times 10^{3}\end{array}$ & 421.32 & 0 & $\overline{1}$ & 1 & 1 & 0 & & 11 & Center of aperture \\
\hline C & $\begin{array}{l}\text { AO } 590 \mathrm{~J} \\
\text { SERI } \mathrm{BAO} 226\end{array}$ & $\begin{array}{l}\text { Outjoor dry } \\
\text { bulo } \\
\text { temperature }\end{array}$ & $T_{0}$ & FNC (3) & $\begin{array}{l}1.7796 \\
\times 10^{6}\end{array}$ & 2030.4 & 775.68 & -454.3 & 1 & 1 & 1 & 1 & & 11 & North side of garage \\
\hline 0 & $\begin{array}{l}\text { AD } 550 \mathrm{~J} \\
\text { SERI BAO248 }\end{array}$ & $\begin{array}{l}\text { Inside dry bulb } \\
\text { temperature }\end{array}$ & $T_{i}$ & FNC (4) & $\begin{array}{l}1.7971 \\
\times 10^{6}\end{array}$ & 2321.3 & 783.60 & -458.3 & 1 & 7 & 1 & 1 & & 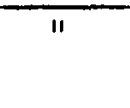 & $\begin{array}{l}51 " \text { from carpet } \\
\text { master bedroom }\end{array}$ \\
\hline E & $\begin{array}{l}\text { AD } 590 \mathrm{~J} \\
\text { SERI } 3 A 0192\end{array}$ & $\begin{array}{l}\text { Inside dry bulb } \\
\text { temperature }\end{array}$ & $T_{i}$ & $\mathrm{FNC}(5)$ & $\begin{array}{l}1.7890 \\
\times 10^{6}\end{array}$ & 2320.5 & 779.74 & -454.4 & 51 & & & 1 & & 11 & $\begin{array}{l}66^{\prime \prime} \text { from carpet } \\
\text { Kelly's bedroom }\end{array}$ \\
\hline$F$ & $\begin{array}{l}\text { AD } 590 \mathrm{~J} \\
\text { SERI } 3 A 0144\end{array}$ & $\begin{array}{l}\text { Inside dry bulb } \\
\text { temperature }\end{array}$ & $T_{i}$ & FNC (6) & $\begin{array}{l}1.7695 \\
\times 10^{6}\end{array}$ & 2321.8 & 770.81 & -450.8 & 01 & & & 1. & & 11 & $\begin{array}{l}89^{\prime \prime} \text { from floor } \\
\text { living room }\end{array}$ \\
\hline G & $\begin{array}{l}\text { AD } 59 D J \\
\text { SERI } \quad \text { BAO24I }\end{array}$ & $\begin{array}{l}\text { Inside dry bulb } \\
\text { temperature }\end{array}$ & $\mathrm{T}_{\mathrm{i}}$ & FNC(7) & $\begin{array}{l}1.7788 \\
\times 10^{6}\end{array}$ & 2320.9 & 775.16 & -454. & 21 & $1 i$ & & 1 & & "I & $\begin{array}{l}\text { Air temperature } \\
\text { at ridge line }\end{array}$ \\
\hline HI & $\begin{array}{l}\text { no } 590 \mathrm{~J} \\
(\mathrm{no} \quad \mathrm{ID} \text { tag })\end{array}$ & $\begin{array}{l}\text { Inside dry bulb } \\
\text { temperature }\end{array}$ & $\mathrm{T}_{i}$ & FNC (8) & $\mid \begin{array}{l}1.7771 \\
\times 10^{6}\end{array}$ & 2320.6 & 774.52 & -453\}$. & 31 & 11 & 11 & 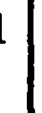 & & 11 & $\begin{array}{l}\text { Air temperature in } \\
\text { ducts below slab }\end{array}$ \\
\hline
\end{tabular}




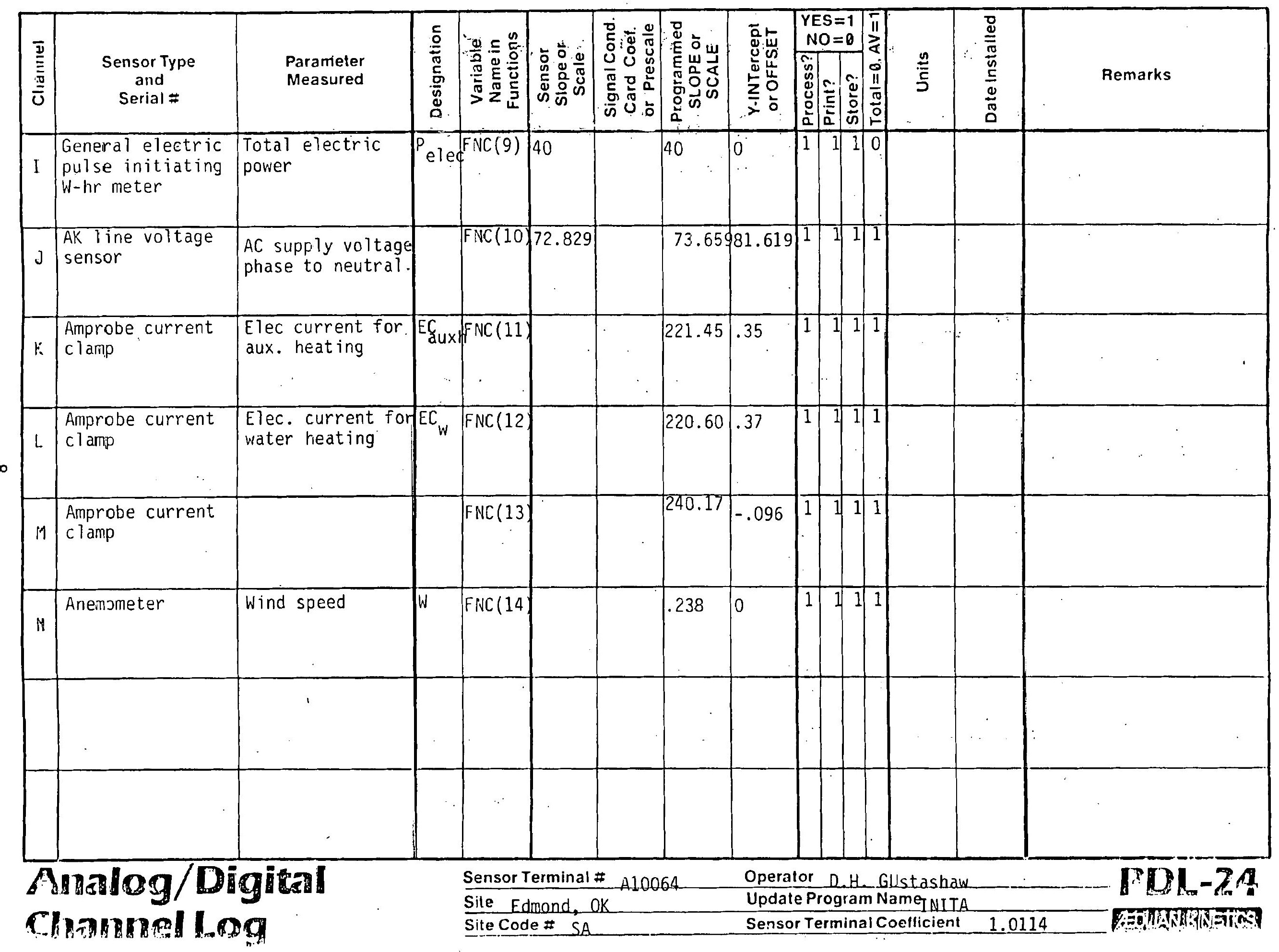




\section{Stadens Chammel Log}

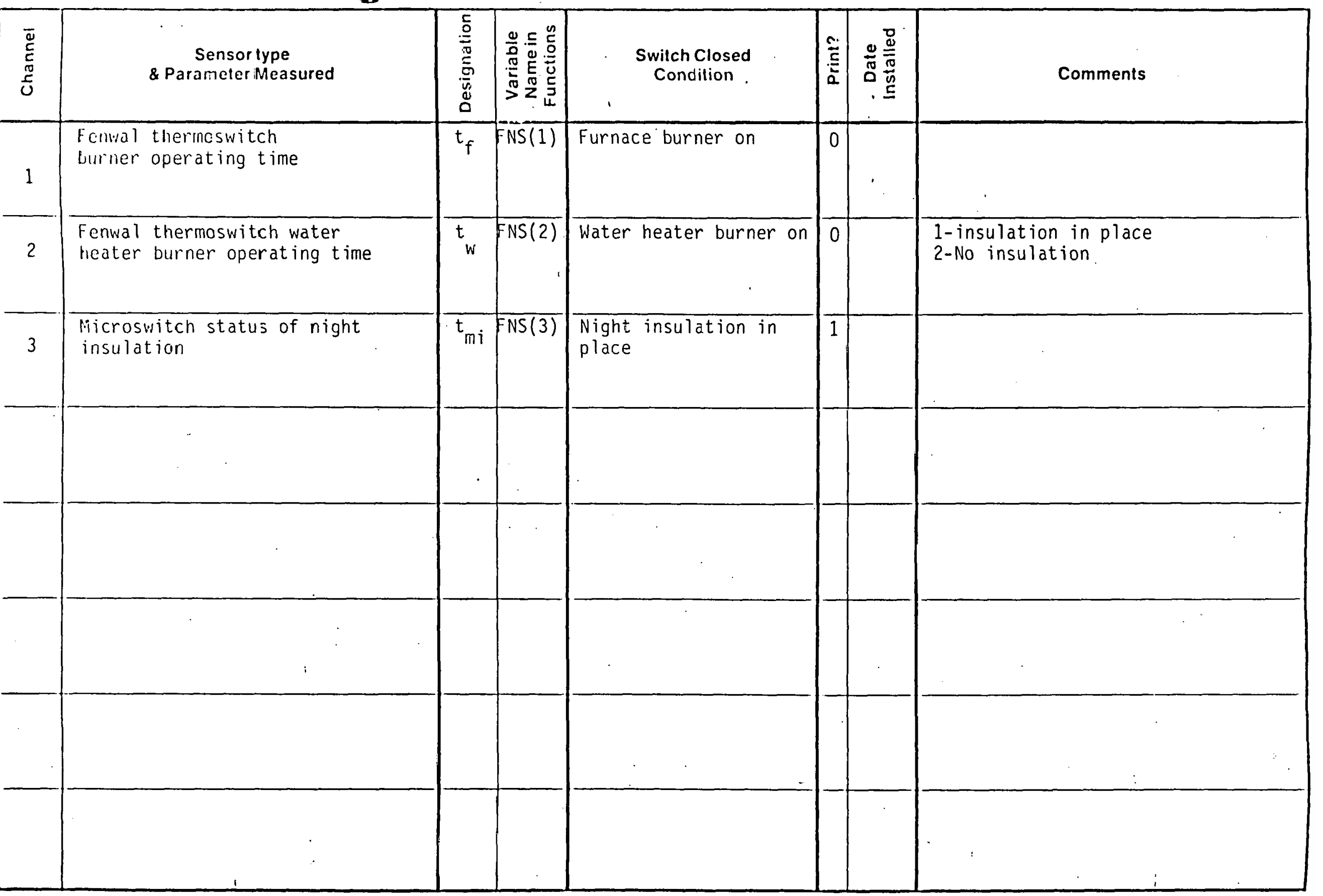


INIT A

$1 / 13 / 83-6 / 9 / 83$

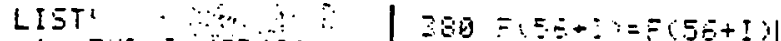

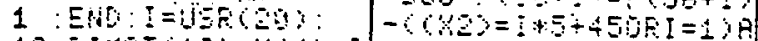

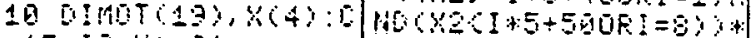
$1=15-5: \because=01$

15 FOE $=255 T 1=T E F-$

4:FOKEABOE I I NEKT

20 OT $(9)=50: 0 T(2)=$

1:0T $(3)=.75$

30 OT $(4)=0:$ OT $(5)=0$

$O T<6\}=0$

40 OTC $7=06:$ OT $(8)=34$

$8: 0 T(9)=2$

58 OTC10\%=6B: OTC11)

$=75: 0 T(12)=440$

60 OT $(13)=77: 0 T<14$

$=2: 0 T(15)=2$

70 OT $(16)=\frac{1}{2}: \operatorname{OT}(17)=$

80 OT $(18)=0$ :0T $(19)=$ 0

$99: I=U 58(24)$

-110 IFY $30-1$ THENISO

120 FORI=1T015: F(I)

$=0:$ NEXT

$140: 34=0:-3=0: 3=0$

$450 \quad \therefore B=(F N C 64+F M C$

5) +FHE(E)

$15: \quad \times 1=F M O 4: x 2=F$ Cद.

$155=2=2+2$

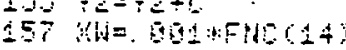

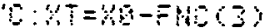

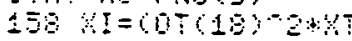

$+60 \mathrm{~T} 1 \mathrm{y}$ )

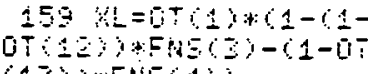

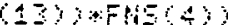

150 IFXTYGTHENA $Q 4$ )

$F(4)-3+3) x\}$

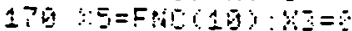

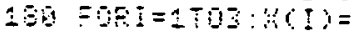

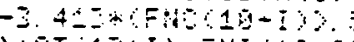

) *

*

$185 \%=R+2(1) N E$

$195=(E)=F(E)+B C$

OT $(5)+10 T(4)+F R E C 4)$

$195 \quad 36=x(2)+10 T(7)+0$

TCE)+FHEOZ+C:KT=3.

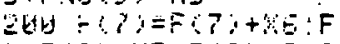

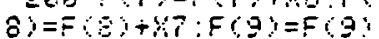

$+x 63$

$240=(5)=F(\theta)+F(7)-$

$F(\theta)+\bar{r}(\bar{\theta})$

$25=2)=$ IT 2 ) $F$ C

280 IFYTSGHEIIT S

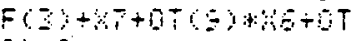

())

$300=1)=F+1-F C O-$

$F 2$

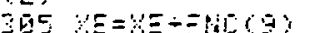

$310=(19)=510)+742$

$3: F A=611 \% 4$

$-10+30+3$

$215=0=1=0-17:=6$

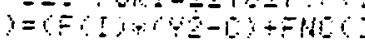

- $30 \mathrm{COT}$

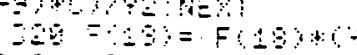

$2-1+6$

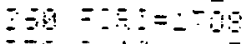

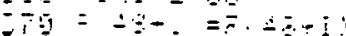

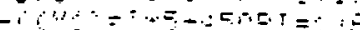

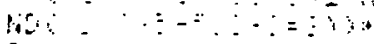

E

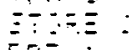

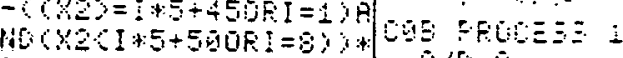

$390 \mathrm{NEKT}$

$410 \quad \times 4=84+35 \times 5$

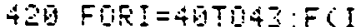

$j=F(I)+F B S C I-35)$ i E.XT

430 FOEI $=44 T 045 \cdot F C$

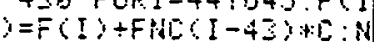

EXT

445 IFFHEC 2$)(0 T(10)$

THEHF $(47)=F(47)+C D T$

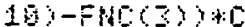

450 IFFMES)DTC11)

THEAF 46$)=F(4 B)+C F M L$

(3)-0T(11)?

460 IFF= TTHEHSOG

470 IFYEYF (ES)THEHF

$655)=K E: F(65)=0: F(E)$

$i=\mathrm{H}$

480 XE $Y E$

560 IFD= 1 THEN999

519 IF $=4$ THEMH

$4=01000$

$520 \quad 1=0: 4=15+12$

520 FGFI $=25 T O 1: F$

$=F(I)+F(I-2 I): H E Y T$

$54 F(1)=F)=(19)+F(1$

$\therefore F(Z)=F(Q 1)+F(2)=$

$23=(23)+5)$

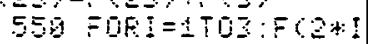

$+18)=F(2 * I \div 17)(F)$

TC: $:$ HEYT

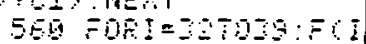

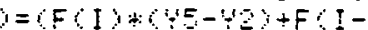

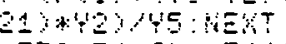

570 F $C 45)=C(45)+C^{4}$

$-12+4+3=$

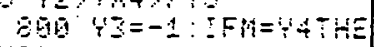
1595

O1日 FrIMTITASGOA"A ORTH"A4"FE RTOUT"

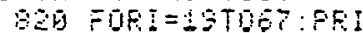

HT! "E I I F CI :FCI $)=0$

MEYT

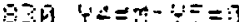

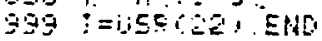

Ffoló !

20

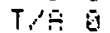

STOEE 1

FP: 1

SLOFE $\because-1 N T$

COC FFICESE 1

H. $\mathrm{C}$ a

$T A 1$

STUEE 1

PRT 1

SLOFE 775.63

Y'IRT -454.

COO FROEES 1

A.

$T: 1$

STORE 1

FET 1

SLOFE 780 5

YTITT -458

OES PROEES 1

606

TS

STOE I

FET

S195 77973

$Y-I B T-4 \bar{T}+48$

DOF FROE:E I

A.

i,

GTOE:

Fet

STE

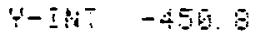

60 $9000=1$

$\therefore \mathrm{D}$

T:B 1

GTOEE I

FE: 1

SLOFE TTE.15

$Y-I N T-454.23$

COAH FETEEEZ 1

Ail 1

T.F 1

STRE 4

FFT 1

SLFE

Hä la $=8 \mathrm{C}$

HA ETRE IHETHL

HES 1 MUll is ?

FURE ETOEE INTEEUE

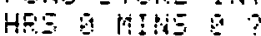

FUMO EEFO TATEEURL

HES OMIPS 5

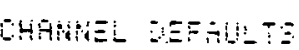

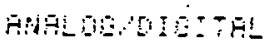

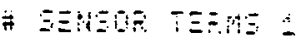

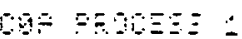

$-8 \mathrm{O}$

$T=$

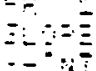

COI FOOSE:

Ad 1

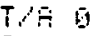

STOFE

FET:

$50=4$

Coj $j=05: 1$

406

$5 \div \div$

50

$F-$

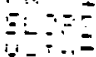

$\because \div \vdots=$
$00=00=2$

A 5

$T \div \div$

$5+10$

Ff:

政

Co $000 \equiv=1$

A 8

$T=$

GP:

Fei 1

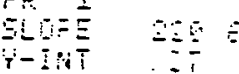

Con $F$ DOSE $=1$

Bi, 1

$T$,

STCE:

FeT:

500217

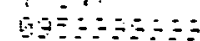

$064=0,0=5$

$\therefore \leq$

ETOF:

Fe?

殉-

UF:

sitis

$50=-$

$58=5$

$50=5$

$50 \div$

$545=0$

$50=5$ i

$5070 \overline{0}$

$50=-5$

UFDF:

ouTP!:

\# QuTFUT

UPCETE 
FURETION DEFTULTE

\# FUNCT DONE 67

F I ETOFE

$F=$ SOFE

$F$ STOE

$F+$ TLES

F 5 Trfe

F $E$ STOE

F 7 ETURE

F $\theta$ STRE G

F 9 TIRE

F10 STOFE

F11 STORE

F12 ETURE 0

FIS STORE E

Fit STORE 6

F15 STURE

F16 STORE

Fí STURE B

F15 STURE 0

F13 STURE 0

FQO STORE E

F21 STORE 0

F22 ETUFE

FES STLRE E

F24 BTUFE O

F.5 STOFE 8

F.E STLRE

F27 STOFE 0

F28 ETORE E

F2S STQRE है

F3G ETURE

FI STOEE B

FSE ETGRE 0

F3E GTGFE

FE4 GIUEE

FS5 STORE 6

F36 STERE

F37 5 TIRE

F3B STURE E

F39 STOFE

FAO STURE

FA1 ETERE O

F42 STOFE

F4S STUEE 0

F44 STURE O

F45 ETIRE

F45 STURE Bิ

F4T ETUFE 0

F48 STORE 0

F49 STORE O

F5G STORE O

F51 STORE O

F52 STURE A

F5S STUFE 0

F54 STORE

F55 STURE B

F.5 STORE B

FST ETURE O

FSO GTURE 0

F59 STURE B

FGQ ETLPE $B$

F51 ETSFE O

FD2 STLFE O

FSS STURE O

FSA STOEE मे

F55 ETOEE O

FOS STLED

Fo'

FFT 1

FE:T 1

FET 1

FERT 1

FET 1

FERT 1

FRT 1

FETT 1

FFT 1

FFET 1

FRT 1

FRT 1

FRT 1

FET 1

FRT 1

FRT 1

FFT 1

FET 1

FFT 1

FRT 1

FPT 1

FRT 1

FRT 1

FPT 1

FPT 1

FFT 1

FET 1

FFT 1

FET 1

FFT 1

FRT 1

FRT 1

FRT 1

FET 1

FRT 1

FET 1

FRT 1

FRT 1

FRT 1

FET 1

PRT 1

FRT 1

FRT 1

FRT 1

FFT 1

FRT 1

FET 1

FRT 1

FRT 1

FRT 1

FRT 1

FRT 1

FRTT 1

FRT 1 .

FRT 1

FRT 1

FRT 1

FRT 1

FRT 1

FRT 1

FRT 1

PRT 1

FPT 1

FRT 1

FRT 1

ERT 1

=RT 1 


\section{SITE SB}

Mr. \& Mrs. Edward Crisp

14807 West Bend Drive

Houston, TX 77082

INIT2 Software Installed: $1 / 18 / 83$ 


\section{SITZ SUMMABY SHEET}

Site Identfication Number SB1
Inst. Contr. Name. ESG, Inc.

Address 2231 Perimeter Park

Suite 11 At lanta, GA 30341
Location:

Town Houston. IX

Latitude $29^{\circ} 4^{\prime}$

Longizude $95^{\circ} 2^{\prime}$

Elevation $50^{\prime}$

Nearest Neather Staclon:

Houston. IX
Building:

Type of Building

General Type of Construction

Ground Coupling

Passive/inybrid jystem(s):

Dominant Heating System Type

Dominant Heaz1ng System Area

Other System Type

Oeher Syscen Area

Nizhe Insulation

Auxiliary Heating System:

systeg and Discribution iype

Day Theranstat jetsing

Vight Theraostat Seting

Liling Zone Eloor Area

rassina tuercure ara

juss Coeszictenz and Dace Yessuzed

In:inzicion laze und Caca :eusured
Two story residence

wond frame and veneer

Slab on grade

Direct gain
$\frac{156 f+2}{N / A}$
$N / A$
$N / A$

Gas forced air $68^{\circ} \mathrm{F}$ $68^{\mathrm{F}} \mathrm{F}$

$2108 \mathrm{ft}^{2}$

$156 \mathrm{ft}^{2}$

$L=224.7$ Btw/hr of $1 / 16$ and $1 / 17 / 83$

No pressure $1 / 15 / 83$ 


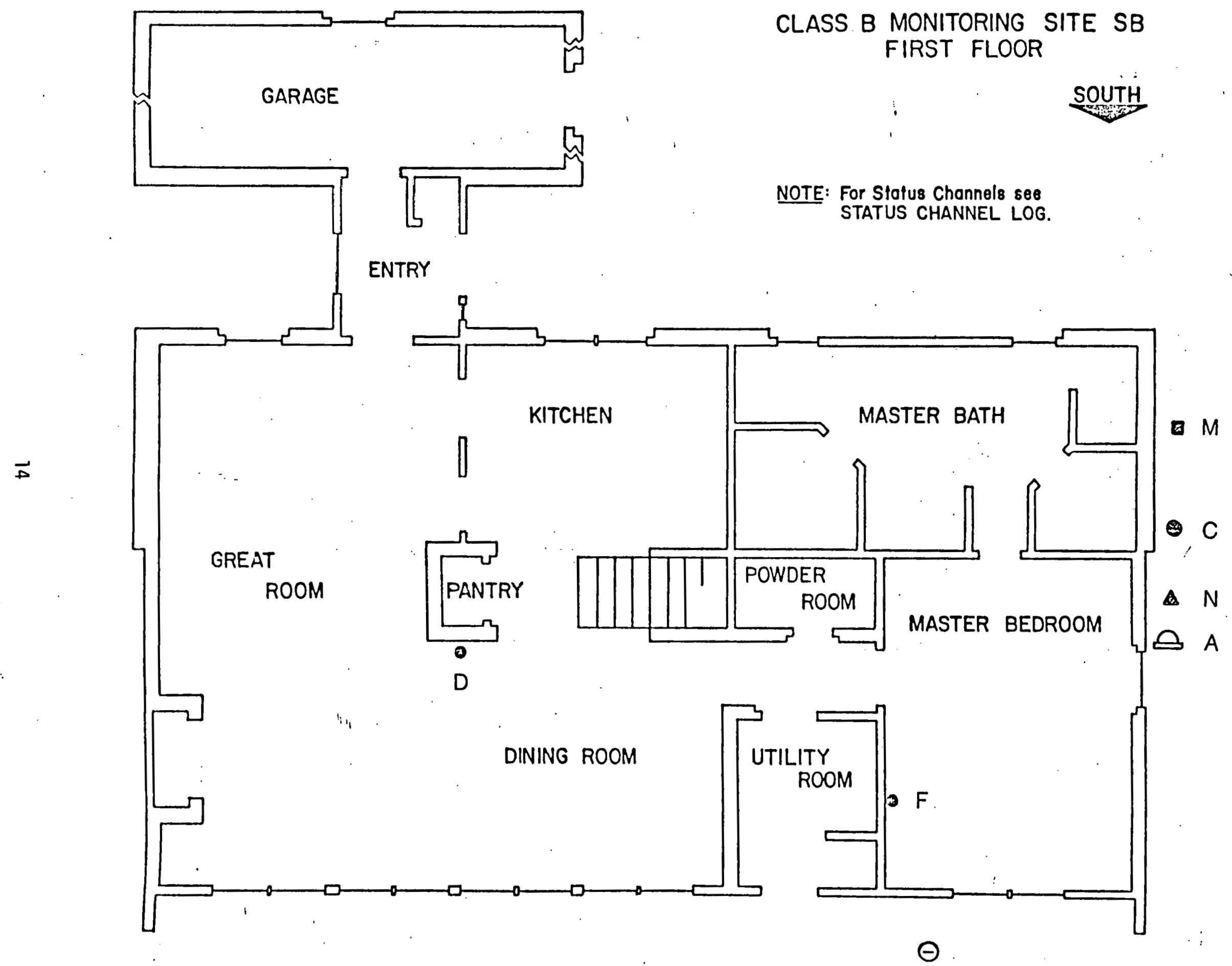


LEGEND

ANEMOMETER

WATT HOUR METER

TEMP SENSOR

VOLTAGE SENSOR $\square$

AMP CLAMP

PDL-24

PYRANOMETER

$\Delta$

$\Theta$

范

\%
CLASS B MONITORING SITE SB SECOND FLOOR

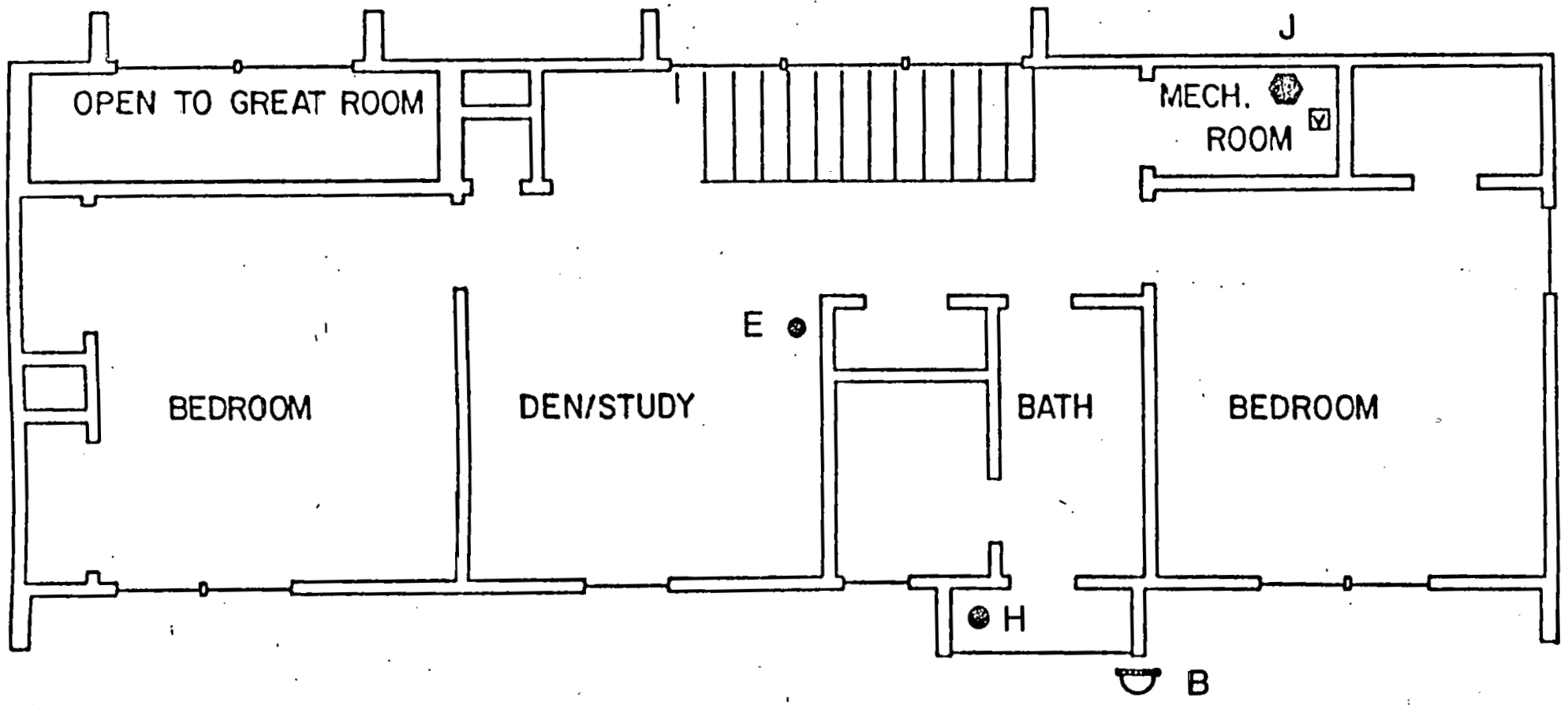


Overall heat loss coefficient

Furnace delivery

efficiency

Air infiltration rate

Furnace burner fuel

flow rate

Heating value of

OT (5) furnace fuel

OT(6)

Water heater burner

fuel flow rate

Heating value of water

OT(7) heater fuel

Water heater jacket

OT (8)

OT(9)

heating factor

Water heater space

heater factor

OT(10) Aux. heating set

point temperature

Aux. cooling set

OT(11) point temperatura

liet passive collector

$0 \mathrm{~T}(12)$

area

$0 T(13)$ iloveable insualtion correction factor

Elec. aux. heating

OT (14) voltage multiplier

Elec. water heating

oT (15) voltage multiplier

Channel If voltage

OT(16) Inultiplier

oT(17) Channel N voltage

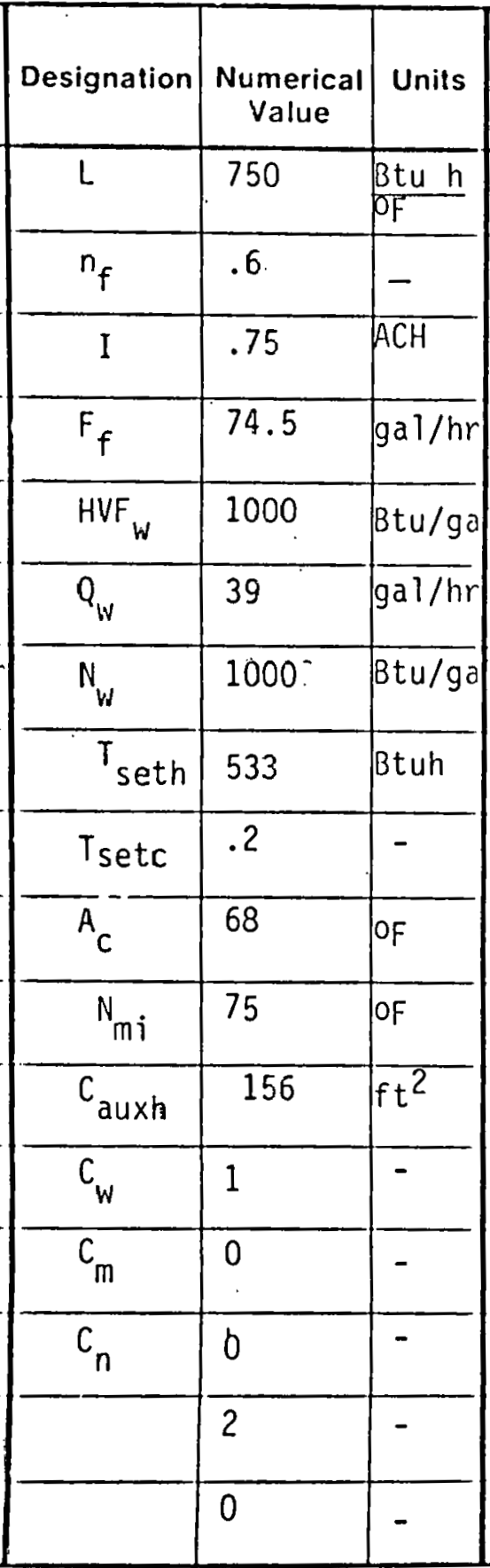

Date INiti

\begin{tabular}{|r|}
\hline Line \\
\hline \\
\hline \\
\hline \\
\hline
\end{tabular}

\section{Program}

Note: Site.was operated with analytical UA instead of coheat value. Stabilization was in question 


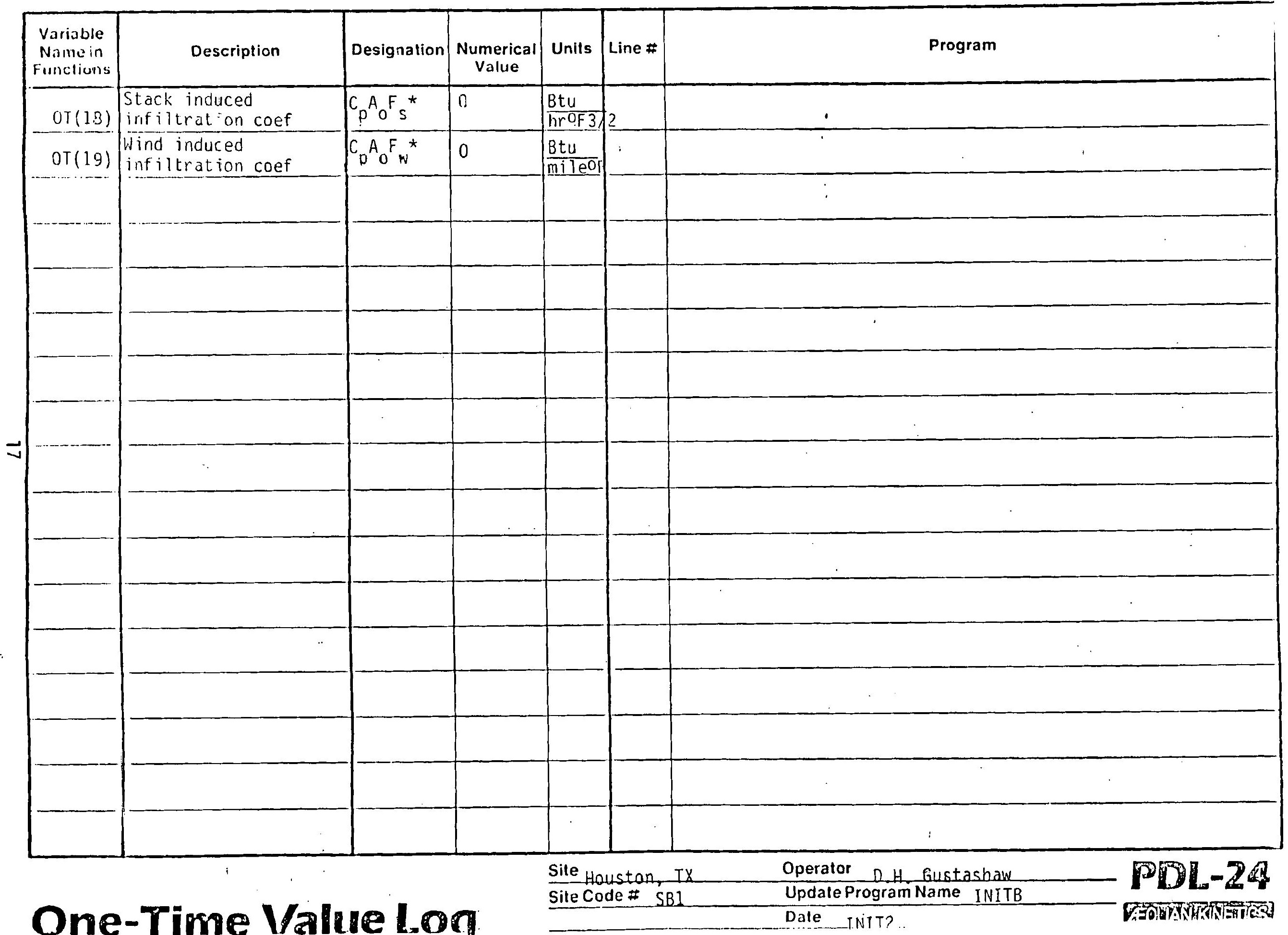




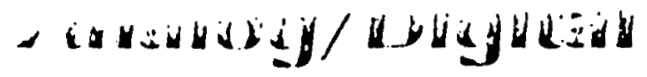 Chandiallog}

\begin{tabular}{|c|c|c|c|c|c|c|c|c|c|c|c|c|c|}
\hline 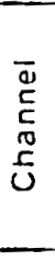 & $\begin{array}{l}\text { Sensor Type } \\
\text { and } \\
\text { Serial }\end{array}$ & $\begin{array}{l}\text { Parameler } \\
\text { Measured }\end{array}$ & 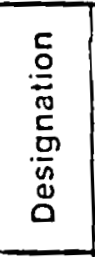 & 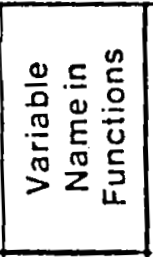 & 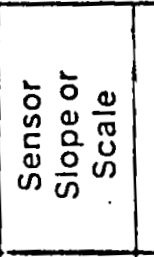 & 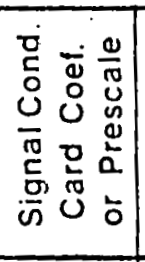 & 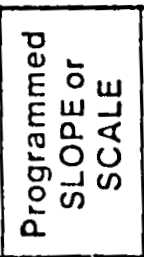 & 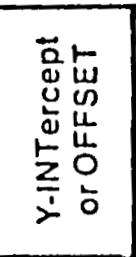 & 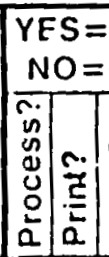 & \begin{tabular}{l|l}
$=1$ \\
0 \\
0 \\
0 \\
0 \\
0 \\
0 \\
0 \\
0
\end{tabular} & 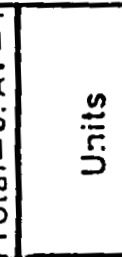 & 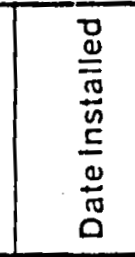 & Remarks \\
\hline A & $\begin{array}{l}\text { 1. icor pyranonter } \\
\text { 19y } 21355\end{array}$ & $\begin{array}{l}\text { Incident solar } \\
\text { radiation on } \\
\text { the horizontal }\end{array}$ & $I_{h}$. & FNC (1) & $\begin{array}{l}4.2351 \\
\times 10^{6}\end{array}$ & 9960 & 431.33 & 0 & $\begin{array}{lll} & 1 \\
\end{array}$ & 10 & & $12 / 81$ & East peak \\
\hline$B$ & $\begin{array}{l}\text { LiCor pyranometer } \\
\text { PY } 2786\end{array}$ & $\begin{array}{l}\text { Incident solar } \\
\text { radiation on } \\
\text { ccllector plane }\end{array}$ & $I_{\text {qn }}$ & FNC (2) & $\begin{array}{l}5.5847 \\
\times 10^{8}\end{array}$ & 9969 & 568.27 & 0 & \begin{tabular}{l|l|}
1 & 1 \\
\end{tabular} & \begin{tabular}{|l|l}
1 & 0 \\
\end{tabular} & & $"$ & Center of aperture \\
\hline$c$ & $A D \overline{\partial g() J}$ & $\begin{array}{l}\text { Outdoor dry bulb } \\
\text { temperature }\end{array}$ & $T_{0}$ & FNC(3) & $\begin{array}{l}1.7792 \\
\times 10^{6}\end{array}$ & 2320 & 777.94 & -454.2 & \begin{tabular}{|l|l|}
1 & 1 \\
& 1
\end{tabular} & \begin{tabular}{|l|l}
1 & 1
\end{tabular} & & $"$ & East side of house \\
\hline v & 10590]$. & $\begin{array}{l}\text { Inside dry bulb } \\
\text { temperature }\end{array}$ & $\overline{T_{i}}$ & FNC (4) & $\begin{array}{l}1.7906 \\
\times 10^{6}\end{array}$ & 3320.4 & 782.09 & -457.5 & \begin{tabular}{|l|l|}
1 & 1 \\
\end{tabular} & \begin{tabular}{l|l}
1 & 1
\end{tabular} & & $"$ & $\begin{array}{l}67 " \text { from living } \\
\text { room floor }\end{array}$ \\
\hline $\mathrm{E}$ & AD $E 90 \mathrm{~J}$ & $\begin{array}{l}\text { Inside dry bulb } \\
\text { temperature }\end{array}$ & $T_{i}$ & FNC (5) & $\begin{array}{l}1.7657 \\
\times 10^{6}\end{array}$ & 2742.6 & 652.38 & -449.5 & 111 & $1 / 1$ & & 7 & $\begin{array}{l}68^{\prime \prime} \text { from floor } \\
\text { upstairs-middle bedroon }\end{array}$ \\
\hline $\mathrm{F}$ & AD $590 \mathrm{~J}$ & $\begin{array}{l}\text { Inside dry bulb } \\
\text { temperature }\end{array}$ & $T_{i}$ & FNC (6) & $\begin{array}{l}1.7750 \\
\times 10^{5}\end{array}$ & 2732.5 & 6588.24 & -452.1 & \begin{tabular}{ll|}
1 & 1 \\
\end{tabular} & \begin{tabular}{|l|l|}
1 & 1 \\
\end{tabular} & & $\pi$ & $\begin{array}{l}69 " \text { from floor } \\
\text { master bedroom }\end{array}$ \\
\hline$G$ & $\overline{A D 590 J}$ & & $T_{S}$ & $F N C(7)$ & & & & & 91 & \begin{tabular}{|l|l|}
1 & 1 \\
\end{tabular} & & " & \\
\hline H & AD 593J & & & FNC (8) & $\left|\begin{array}{l}1.7786 \\
\times 10^{6}\end{array}\right|$ & 2320 & 776.98 & -455.8 & 11 & \begin{tabular}{|l|l|l}
1 & 1 \\
1
\end{tabular} & & " & $\begin{array}{l}\text { Passive water } \\
\text { heater room }\end{array}$ \\
\hline
\end{tabular}




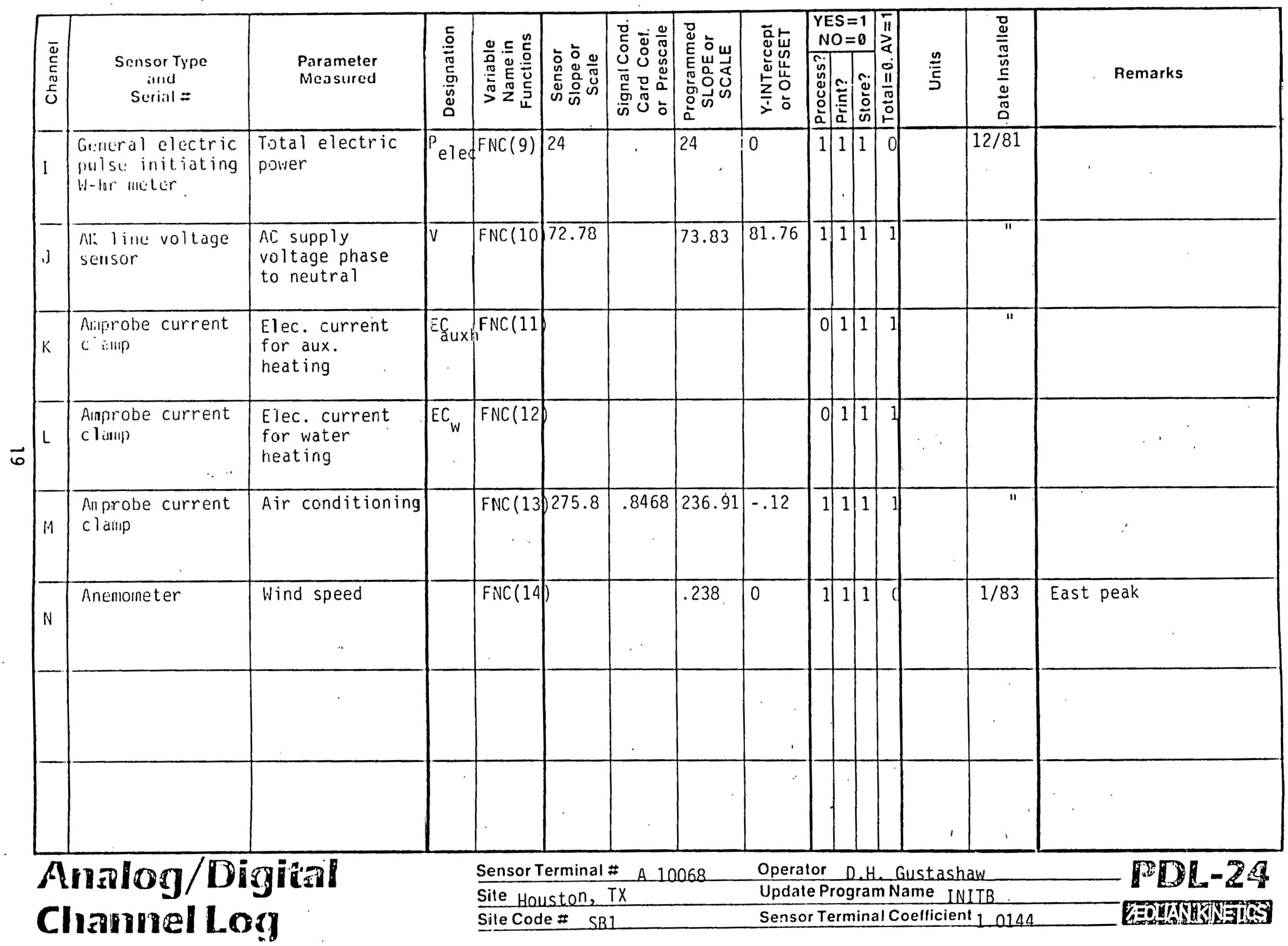




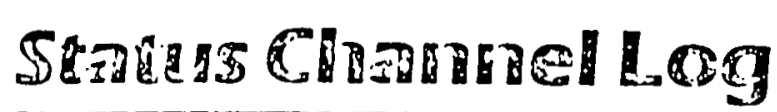

\begin{tabular}{|c|c|c|c|c|c|c|c|}
\hline $\begin{array}{l}\overline{\mathrm{v}} \\
\mathrm{c} \\
\bar{c} \\
\tilde{v}\end{array}$ & $\begin{array}{c}\text { Sensor type } \\
\text { \& Parameter Measured }\end{array}$ & 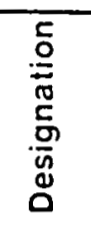 & 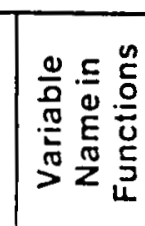 & $\begin{array}{l}\text { Switch Closed } \\
\text { Condition }\end{array}$ & : & 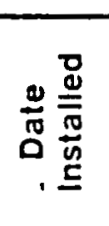 & Comments \\
\hline 1 & $\begin{array}{l}\text { Femval thermoswitch furnace } \\
\text { burner operating time }\end{array}$ & $t_{f}$ & FNS (1) & Furnace burner on & 1 & & \begin{tabular}{|l}
-furnace on \\
0 -furnace off
\end{tabular} \\
\hline 2 & $\begin{array}{l}\text { Ferwal thermoswitch water heater } \\
\text { burner operating time }\end{array}$ & $t_{w}$ & FNS(2) & Water heater burner on & 1 & & $\begin{array}{l}\text { 1-heater on } \\
0 \text {-heater off }\end{array}$ \\
\hline 3 & $\begin{array}{l}\text { Microswitch status of night } \\
\text { insulation }\end{array}$ & $t_{m i}$ & FNS (3) & $\begin{array}{l}\text { Night insulation in } \\
\text { place }\end{array}$ & 0 & & \\
\hline 4 & Surner shades on southside & & FNS (4) & & 1 & & $\begin{array}{l}1 \text {-shades up } \\
0 \text {-shades down }\end{array}$ \\
\hline 5 & North clerestory -Group of 3 & & FNS(5) & & 1 & & $\begin{array}{l}\text { 1-windows closed } \\
0 \text {-windows open }\end{array}$ \\
\hline 6 & North clerestory -Group of 2 & & $\operatorname{FNS}(6)$ & & 1 & & $\begin{array}{l}1 \text {-windows closed } \\
0 \text {-windows open }\end{array}$ \\
\hline 7 & Dryer fenwal switch & & FNS(7) & & 1 & & $\begin{array}{l}1-d r y e r \text { on } \\
0 \text {-dryer off }\end{array}$ \\
\hline 8 & Dryer vent position & & FN (8) & & 1 & & $\begin{array}{l}\text { 1-vent closed-winter mode } \\
\text { 0-vent open-summer mode }\end{array}$ \\
\hline
\end{tabular}




\section{INIT B}

$1 / 17 / 83-6 / 9 / 83$

LIST $\therefore \because \because$.

1 :ENO ?=155:20)

10 OIHOT(19) K(4)

$1=0 E-2: 1=1$

15 FOE? =55THI TEP-

4.FOKE 4QBEI, I:MENT

20 OT $4=750:$ OT $(2)=$

$6: 0 T(3)=.75$

30 OT $(4)=74.5: 0 T<5$

$=1000: 0 T(6)=39$

40 0T ( T) $=1000$ :0T $(3)$ $=533$ OT $(9)=2$

50 OT 10$)=58: 0 T(11)$

$=75: 0 T(12)=155$

60 OT $(12)=1: 0 T(14)=$

0:0T:15:=0

$7607(16)=2: 0 T(17)=$

$\theta$ 80 OT $(48)=0)$ OT $(19)=$

SQ: : =USR ( 24$)$

41D IFY3CY-1THEYIE

120 FORI $=1 T 018: F(I$

=0:NEXT

$\therefore 140 \quad Y 4=0: Y 2=0: Y Z=0$

150 KX $=$ CFNC(4)+FNCO

$5)+F+L(5)): 3$

$151: 1=F M C A 4: 32=F M$

c(a)

$155: 2=12+1$

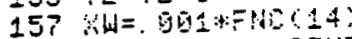
C:XT=YOT-FHSC: IFYT CDTHENKT=

$158 \times 1=60 T 48,-24 \%$

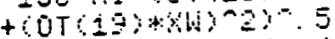

$150 \quad: L=0 T 14: 1-61$

QT $(12)$ ) FWEC 3$)-(1-0 T$ (1) $)=60(4)$

160 FYTYGTHEHFC4:

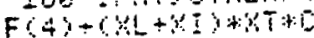

$179 \quad 25=7012,: 93=$

100 FOE $\mathrm{i}=40 \mathrm{~T}$ : $: \mathrm{C} \mathrm{I}=$

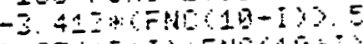

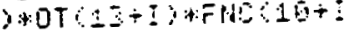

$* 2: 5 * \mathrm{C}$

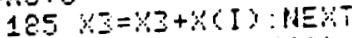

$19 \mathrm{~g}=\mathrm{G})=\mathrm{F}(\mathrm{E}) \mathrm{S}(1)$

OTCS)*UT(4)+FVE(1)*C

$195 \times 6=2(2)+0 T(6)+1)$

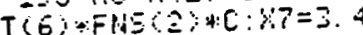

13*FIIC $C:-x 3$

E09 $F(T)=F(7)+B E: F($

$8)=F(8)+K 7: F(\theta)=F(\theta)$

$+34)^{2}=(5)=F(6)+F(7)+$

$F(B)+F(G)$

S5 $=2)=0 T(2)+F(E)$

200 IFUT DTHEPFC

$F(B)+Y+0 T+3)+x=0 T$

E)

$300 F(1)=F(4)-F(2)$

$F(2)$

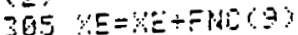

I1日 $F 10)=F 10 \%+F(4)$

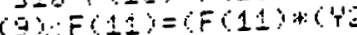

$-8+46)$

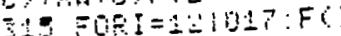

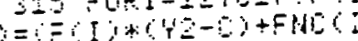

-OWOP NERT

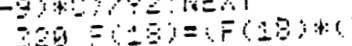

$2-8+300$

$80 \div 6=2 T 08$

$-70=0-1=0+1+1$

$-1+0=0=05=0$

(4)

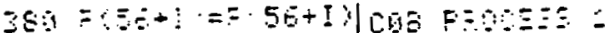

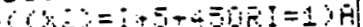

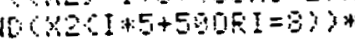

390 HEXT

$410 \quad 34=44+\times 5+5$

420 FOFI $I=40 T 043: F C:$

)$=F(I)+F H 5 C D-25): C: N$

E:TT

430 FORI $=44 T 045: F(1$

$x=F(I)+F H C(I-4))+C: N$

EXT

44Q IFFNICS)COTC10:

THERF $(47)=F(47)+C O T$

10) $-F \mathrm{HCO}(3) \times 4 \mathrm{C}$

450 IFFMCOSOSOTC11

THENG $(4 Q)=F(48)+C F H C$

(3)-UT $(11)) \mathrm{dC}$

46 IFF $=$ STHENSDO

470 IFUE)

$(65)=\mathrm{F}: F(65)=0: F(67$

$\gamma=4$

$450 \quad X E=0$

500 IFD= 1 THENOSP

510 IF:1=1 THEN'1 =0

$44=0.001095$

$520 \div=0: 15=45+12$

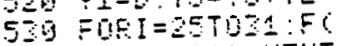

$i=F(I)+F(I-21): M E K T$

$549=(19)==49 y+51$

$F(21)=(21)+F(2) F$

$3)=(23)+3$

SE FOEI = 1 TOS:= 2 I

$+18)=F(2+1+17)$ < $C 25$

+ CA? :HENT

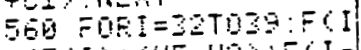

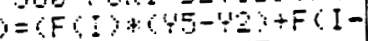

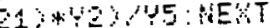

$570=60=6 F(45)+6$

$5-2)+24)$

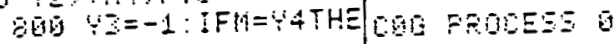

1139

OA EJUTITAECQA" M

OATH"YA "FFIMTOUT"

820 FUEI=1 TOST: FEI

UT: $F " I ; F(I): F(I)=B$ :

HEXT

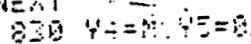

909 $1=5=20010$

$993 \quad I=E R(Z=;:$ ENO

FFigia 1 .

TIUE DEFBC-TS

TEAR 5 ?

FEIHT INTEFUAL

HES 24 WIPS

DH ETOEE IMTEFUAL

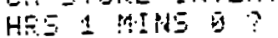

FUNO STOFE IMTEEUTL

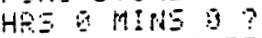

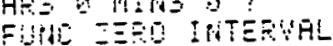

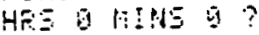

GAHUHE: DEFAUTS

ARALOSOIDT THL

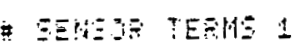

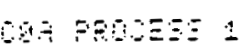

$4 x^{\prime} \mathrm{B}$

T

Exce.

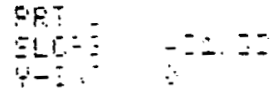

A 0

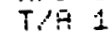

STOFE:

FFT 1

GUFE TTE. 90

YINT -455.34

COI FFGLES 1

AIC I

Tit 0

STORE 1

FET 1

SELE 24

OFFEET 6

COI FEOEES 1

.906

$T: A 1$

ETOEE 1

FET 1

SLOE TS.

$Y$ YIUT $Q L . T E$

Cok FPOCE:

CQ1 20030
A 0

$T, \bar{B}$

ETOFE 1

FFi 1

SLOFE 5ES. 27

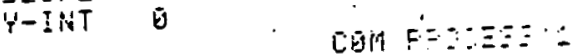

CBC FFCESE 1 ACB

Fis

$T: A 1$

STOFE 1

FFT 1

S10E5 777.54

$i-I B T$-454. 24

CDO PFOCESE 1

A. 0

T.TA 1

STORE 1

FRT 1

SLIFE 782 .

प-IHT -45\%, 54

GE FCOOESE 1

A. 0

$T A 1$

ETOPE 1

ETT 1

SLEE 65230

Y-14T -4.5.5

DF PFOCE:

A. CD

T. 1

$\operatorname{STOES} 1$

PRT:

SLOES BSE. 3

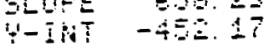

T:A 1

STOEE I

FRT 1

SLAFE 23E.91

प-IHT -.

Can PEOLES:

41

STOES:

FFT 1

$55=5-230$

UFDATE?

STATHS

5 FI FET 1

542 FET 1

$5 \mathrm{~s} F \mathrm{FT}$

STHE

SGE FRT 1

SAE FRT I

S5 FFT 1

595 FPT 1

UPQETE?

OUTFUT

\# OUTFUT TEEHS

UFOATE
SEASON 
FUNETION DEFAISTE

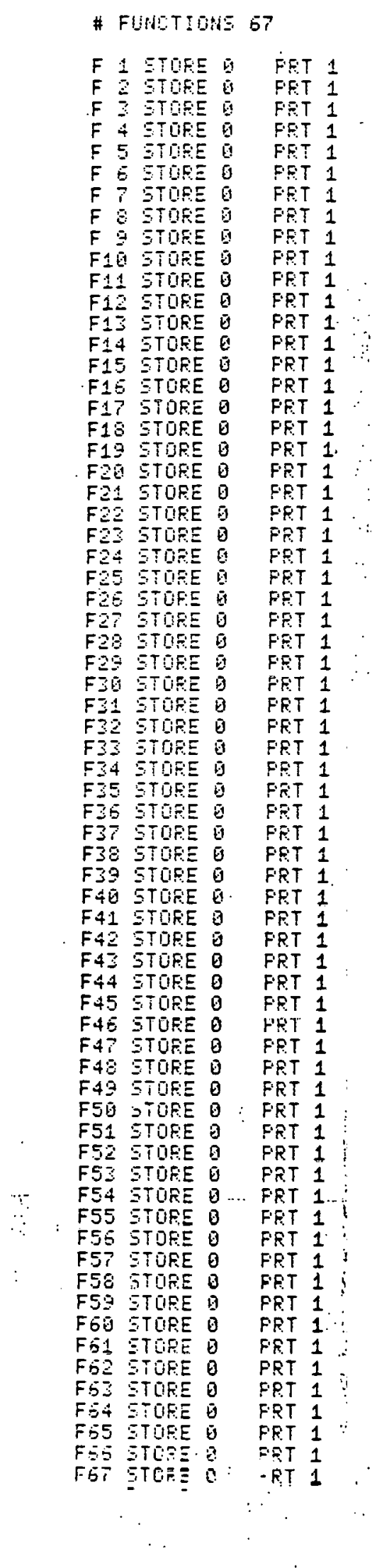




\section{SITE SF}

Mr. \& Mrs. Martin Levin

412 Camp Road

Suwanee, GA 30174

INIT2 Software Installed: 12/30/82 


\section{SITE SUMMART SHEET}

Site Identffication Number SEl
Inst. Contr. Naze ESG, Inc. Address 2231 Perimeter Park

Suite 11 At lanta, GA 30341

Locarion:

Town Suwanee, GA

Latizude $33^{\circ}{ }^{\prime}$

Longi=ude $84^{\circ} 3^{\prime}$

Elevation 1005'

Nearest Neather Starfon:

Atlanta, GA

Buildiaj:

Type of Building

General Type of Construction

Ground Coupling

Two story residence

Wood frame and veneer

Full basement w/partial davlighting

Passive/Aybrid System(s):

Dodiaant Heating System Type

Dominant Heaz1n3 Syscem Area

ot!ue: Sysiem Type

Oeher System Area

Niatic Insulation

Auxiliary Heacing Syscem:

Systez and Jlscribucion Type

Da: Therzostat jetsing

Nizit Therzostat Secting

Direct gain

$158 \mathrm{ft}^{2}$

$N / A$

$N / A$

$N / A$

Eropane:forced air (Tower floor)

Elec. resistance (upper floor) $70^{\circ}$

$70^{\circ}$

Living Zone Floor trez

?assire tuerzure ira

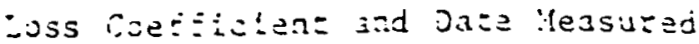

In:ilgition iate lnd jate leusuzed

$2200+2$

$158 \mathrm{ft}^{6}$

$L=604.3 \mathrm{Btu} / \mathrm{hr}$ OF 12/16\% 12/17/82

No pressure 12/17/8? 


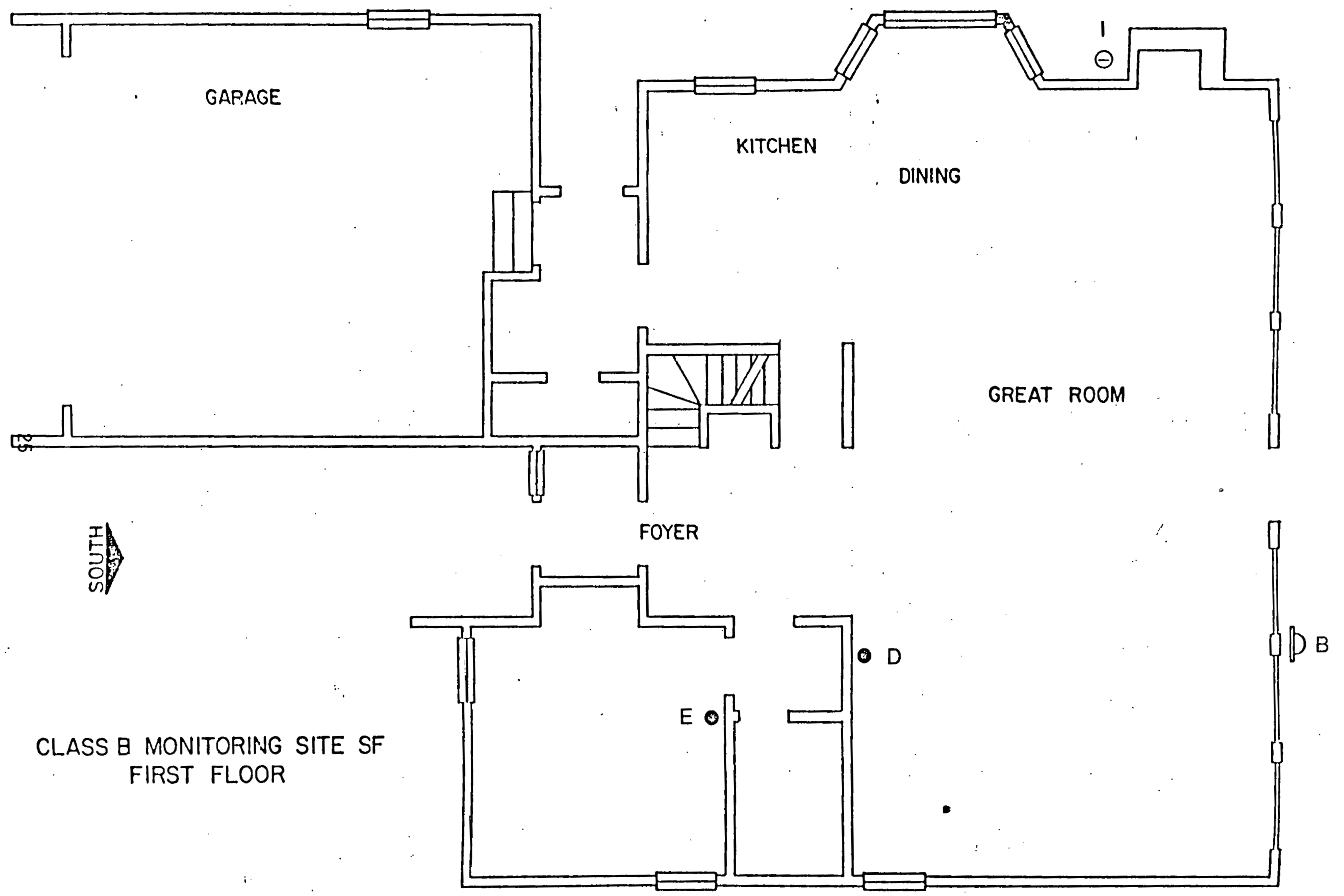




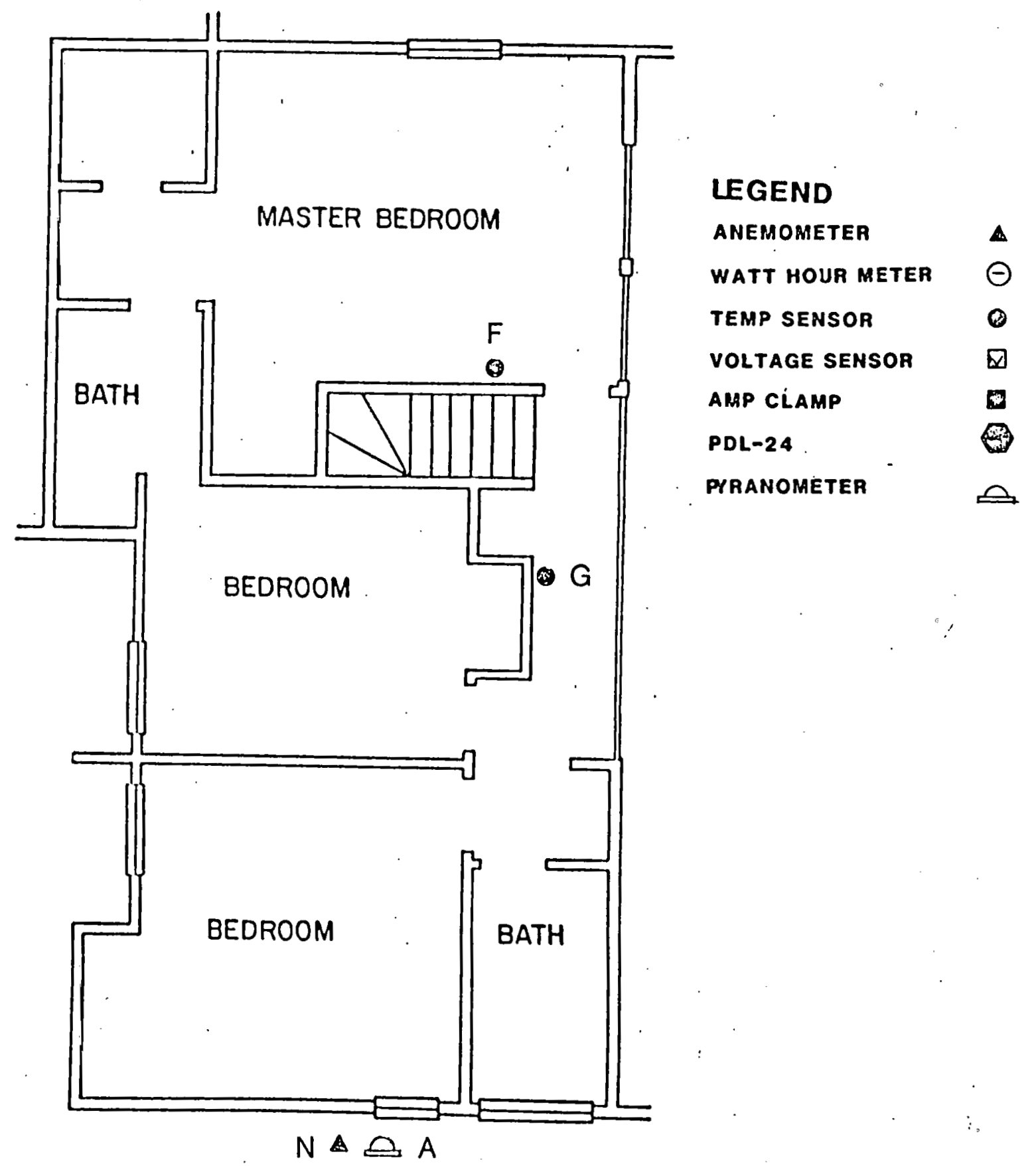




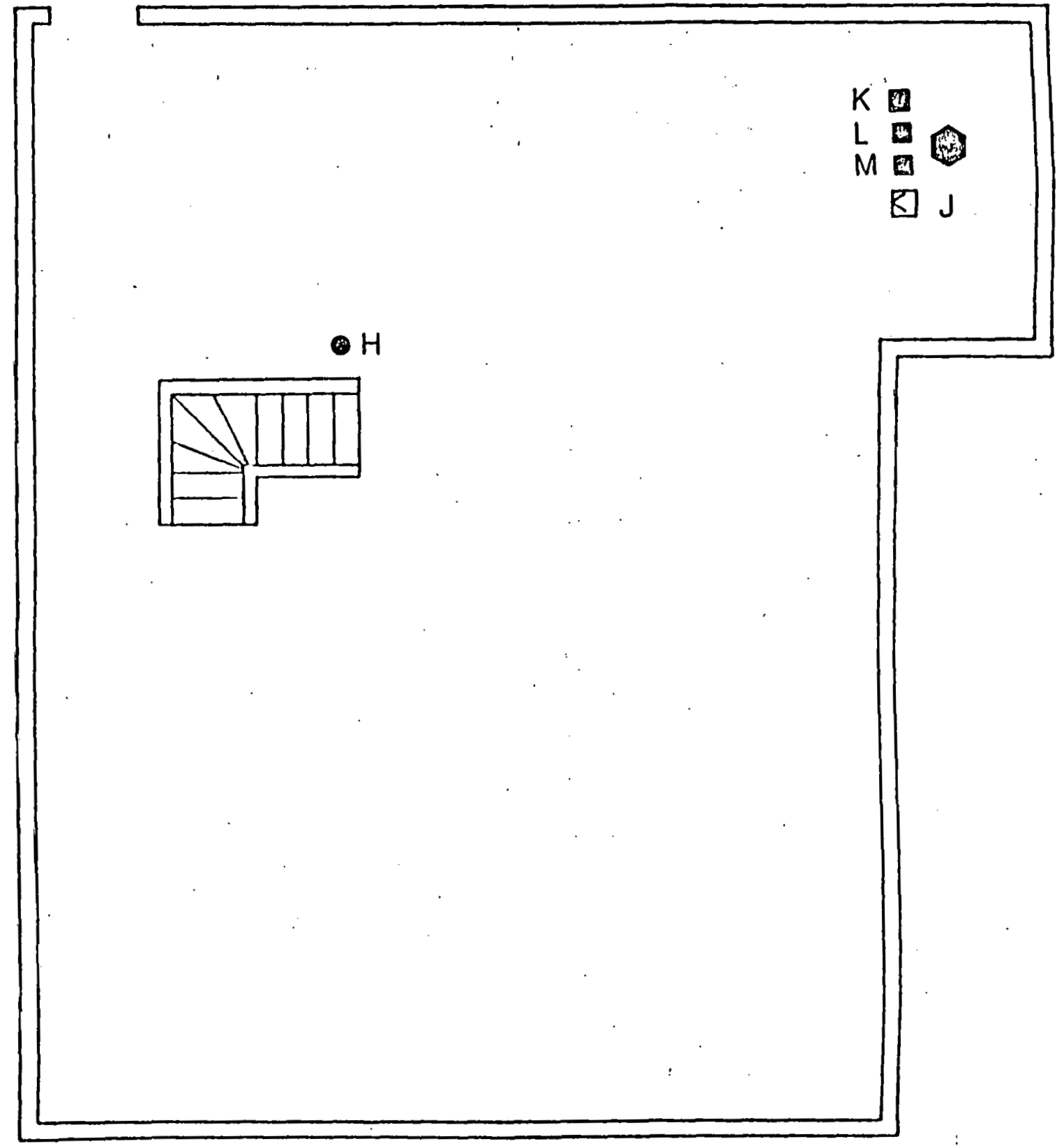


Sile Suwanne, GA

One-Time Valdse Log

\begin{tabular}{|c|c|c|c|c|c|c|}
\hline $\begin{array}{l}\text { Variable } \\
\text { Name in } \\
\text { Functions }\end{array}$ & Description & Designation & $\begin{array}{c}\text { Numerical } \\
\text { Value }\end{array}$ & Units & Line \# & Program \\
\hline$\dot{U T}(1)$ & $\begin{array}{l}\text { Overall heat loss } \\
\text { coefficient }\end{array}$ & L & 604 & $\frac{B t u h}{O E}$ & & \\
\hline $\operatorname{or}(2)$ & $\begin{array}{l}\text { Furrace delivery } \\
\text { cfficiency(propane) }\end{array}$ & $n_{f}$ & .65 & - & & \\
\hline OT $(3)$ & Air infiltration rate & I & .75 & $\overline{\mathrm{ACH}}$ & & \\
\hline OT $(4)$ & $\begin{array}{l}\text { Furnace burner fuel } \\
\text { flow rate }\end{array}$ & $F_{f}$ & 40 & $\mathrm{gal} / \mathrm{hr}$ & & This was computed on the name plate \\
\hline $0 \mathrm{~T}(5)$ & $\begin{array}{l}\text { Heating value of } \\
\text { rurnace fuel }\end{array}$ & $\mathrm{HVF}_{\mathrm{f}}$ & 2000 & Btu/SC & & rating of the propane furnace \\
\hline $0 T(6)$ & $\begin{array}{l}\text { Water heater burner } \\
\text { fuel flow rate }\end{array}$ & $F_{W}$ & 20 & gal/hr & & Based on nameplate of propane water heater \\
\hline OT $(7)$ & $\begin{array}{l}\text { Heating value of water } \\
\text { heater fuel }\end{array}$ & $H V F_{W}$ & 2000 & $B t u / g i$ & 1 & . \\
\hline OT $(8)$ & $\begin{array}{l}\text { Water heater jacket } \\
\text { heating factor }\end{array}$ & $Q_{W}$ & 0 & Btuh & & Modifications to software: \\
\hline OT $(9)$ & $\begin{array}{l}\text { Water heating space } \\
\text { heater factor }\end{array}$ & $N_{w}$ & .1 & - & & $150 \times(0)=(F N C(4)+F N C(5)+F N C(6)+F N C(7) / 4$ \\
\hline $0 \mathrm{~T}(10)$ & $\begin{array}{l}\text { Aüx. heating set } \\
\text { point temperature }\end{array}$ & $T_{\text {seth }}$ & 70 & ${ }^{O} \mathrm{~F}$ & & $\begin{array}{c}189 \times \mathrm{XA}+0 \mathrm{~T}(5) * 0 \mathrm{~T}(4) * \mathrm{FNS}(1) * \mathrm{C} \\
\vdots\end{array}$ \\
\hline OT (11) & $\begin{array}{l}\text { Aux. cooling set } \\
\text { point temperature }\end{array}$ & setc & 75 & ${ }^{O} \mathrm{~F}$ & & $25 r_{2} F(2)=F(2)+0 T(2) \star X A+. .95 \star X(1)$ \\
\hline OT (12) & $\begin{array}{l}\text { Het passive collector } \\
\text { area }\end{array}$ & $A_{C}$ & 158 & $\overline{F T^{2}}$ & & \\
\hline OT (13) & $\begin{array}{l}\text { Moveable insulation } \\
\text { correction factor }\end{array}$ & $N_{m i}$ & 1 & - & & \\
\hline OT (14) & $\begin{array}{l}\text { Elec. aux. heating } \\
\text { voltage multiplier }\end{array}$ & $C_{\text {auxh }}$ & 2 & - & & \\
\hline $0 T(15)$ & $\begin{array}{l}\text { Elec. water heating } \\
\text { voltage multiplier }\end{array}$ & $C_{W}$ & 0 & - & & \\
\hline $\operatorname{OT}(16)$ & $\begin{array}{l}\text { Channel M voltage } \\
\text { lultiplier }\end{array}$ & $c_{m}$ & 2 & - & & \\
\hline OT $(17)$ & $\begin{array}{l}\text { Channel } N \text { voltage } \\
\text { multiplier }\end{array}$ & $c_{n}$ & 0 & - & & \\
\hline
\end{tabular}

Operalor D.th_ Gustashave

Updafe Program Name INITF $151 \mathrm{LL}-24$

Sile Code \# SF 1 C: 


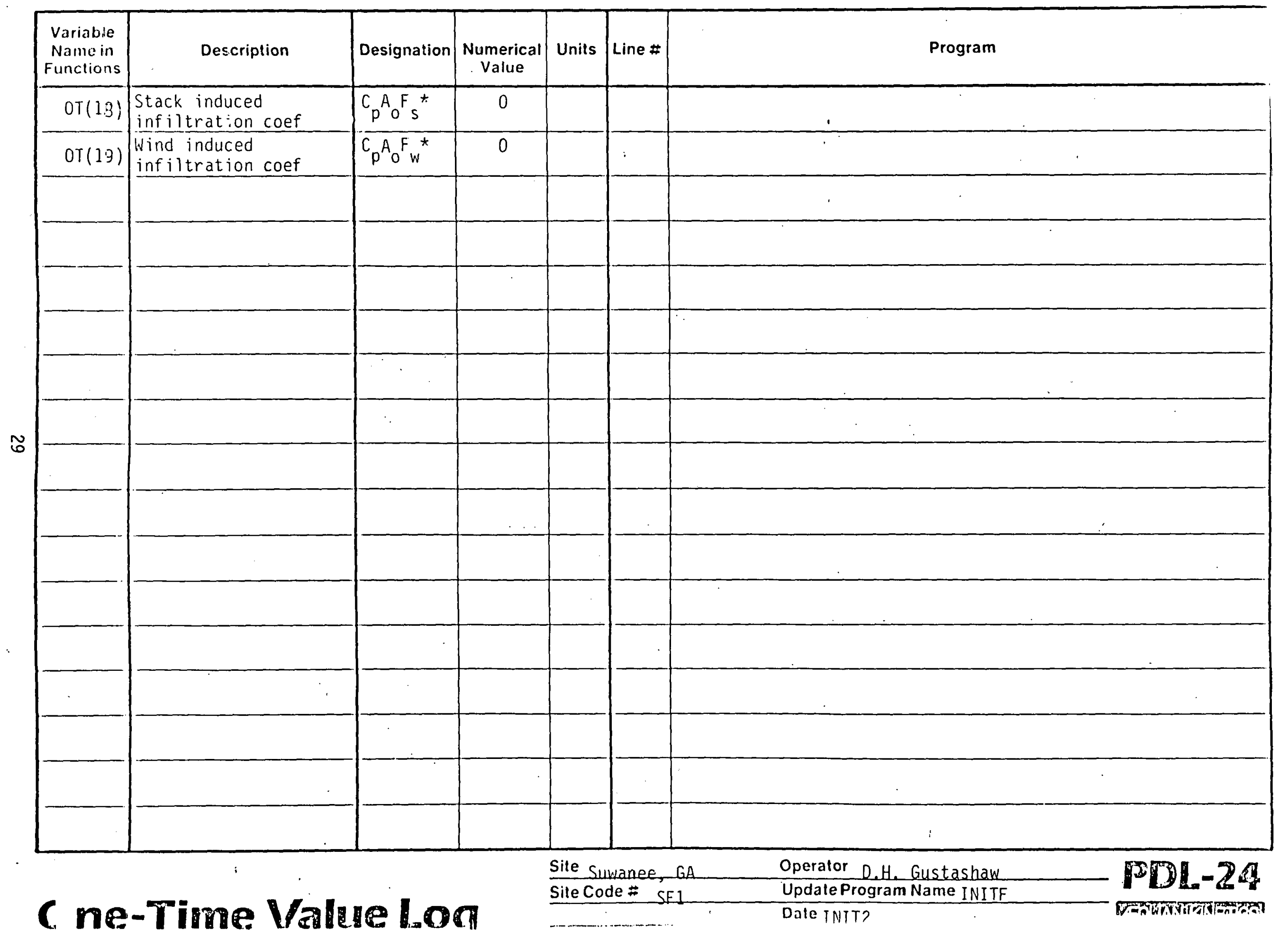




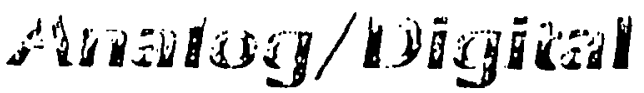

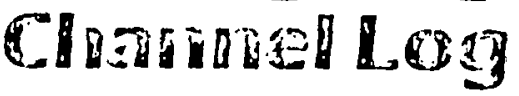

Sensor Terminal $=10113$

Operator D.H Gustashaw

$1810-28$

Site Sulanes Update Program Name INITF Q

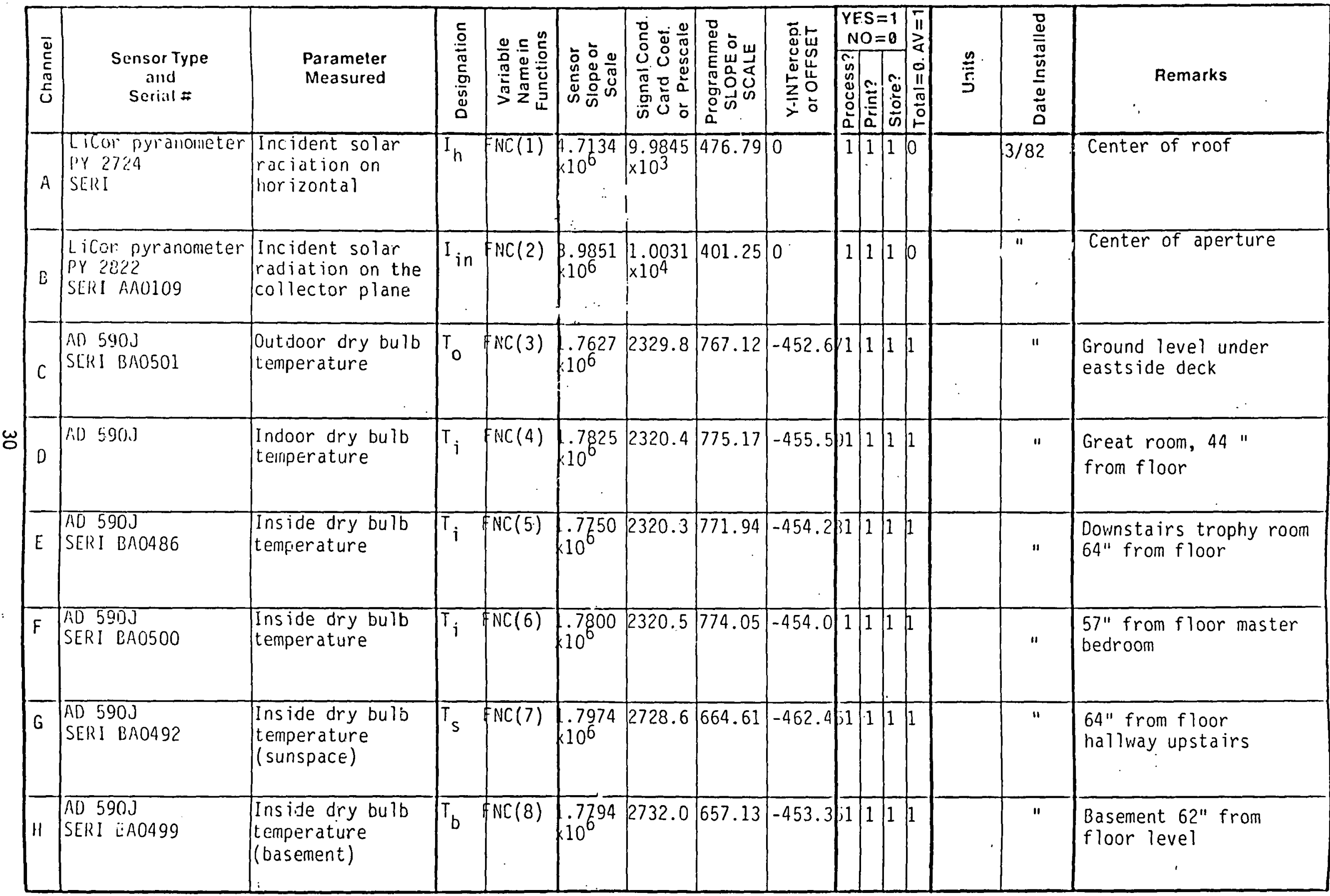




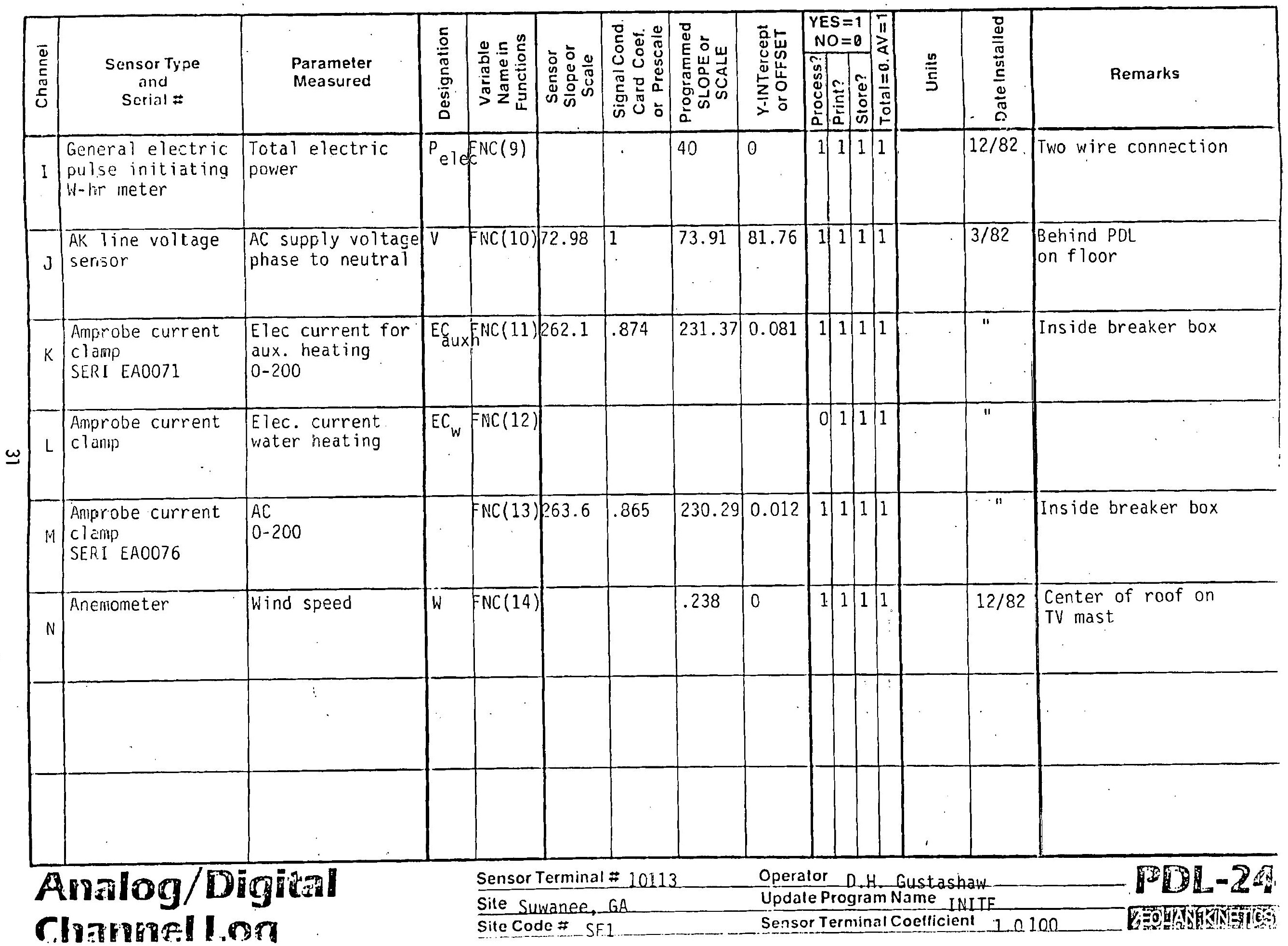




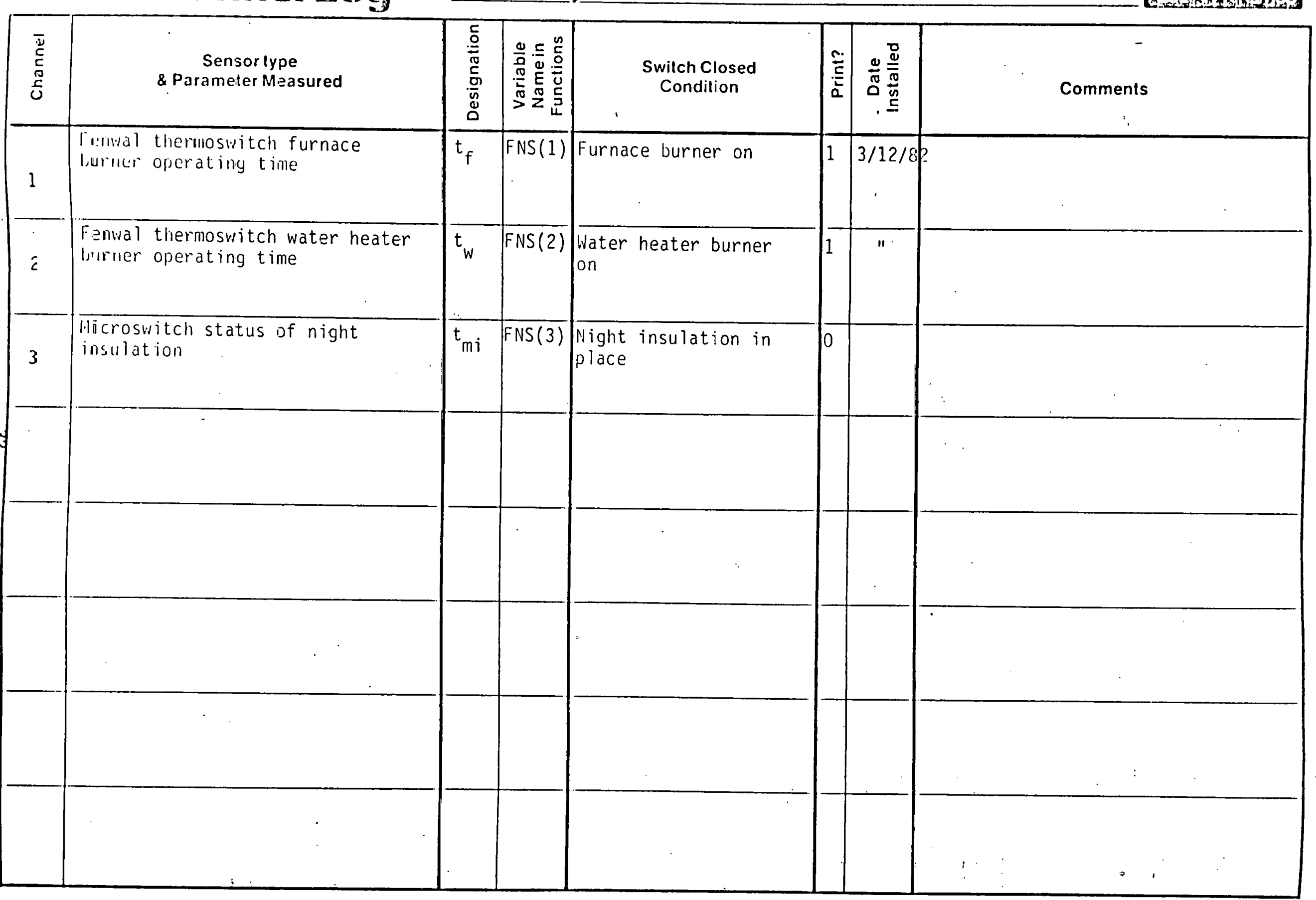


INIT F

LI:5i

$\because$ El: = = 200

-10 010? 193,44$)$

$1=$

A $F$ TRI = E STOLSTEP

4 FUKE4695: I : NEYT

$2007(1)=04: 0 T(2)$

E5:0T $3=75$

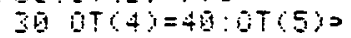

690 : OTCE) $=20$

40 OTC

$=0: 0 \div 69=$

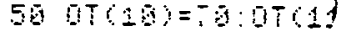

$=75: 0142:=58$

60 0TC1 : $=0 T 64$

2 OT: $=0$ 5

70 OTC1E)

80 OT:A8:=0:0TC19: 5

जa $i=115804 \%$

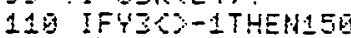
$1 \%$ FLRI=1TOLE:FCI:

$=Q: N E Y T$

$145 \quad x 4=0: 12=0: 43=0$

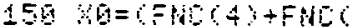

5) +FMCE)+FMC $(7), 4$

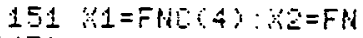

Q67)

$155 \quad 2=0+8$

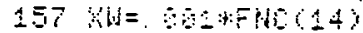

S: $x \bar{i}=40-70 \mathrm{CO}$

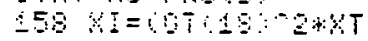

$\div$ - DTCG

$159 \quad 1=0 T-1)(1-C 1-$

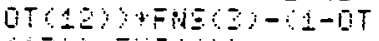

CIZTHFEC

160 IFMTSOTHEHA

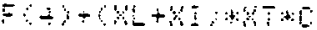

$470 \quad \therefore=F \div-8: 48=0$

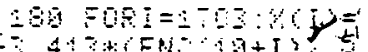

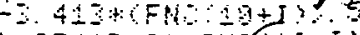

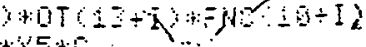

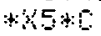

$195,3=83 \div 801)$ NEST

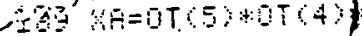

Fis $S, ?+1$

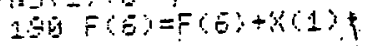
$Y A$

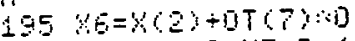

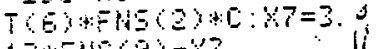

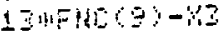

$20 \bar{F} F(7)=F(7)+4 E: F$.

$B)=F(\theta)+Y 7: F(3)=F(\theta)$ $+303$

$24 \mathrm{~A} F(5)=F(E)+F(7)$

$F(\theta)+F(9)$

250 F $2=F(2)+0 T C$

$+5+35+4(4)$

QQD :FXTOETEUF $(3)$

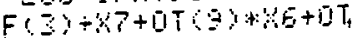

$3 \mathrm{x}+\mathrm{C}$

$305) F(1)=74 ;-7(3)$

F (2)

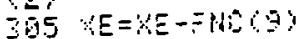

$210 F(10)=F(10)+F 1$

$(9)=11)=6(11)+6$

$-1)+84+1)$

$35=0 \mathrm{I}=\mathrm{T}=1 \mathrm{~T}: \mathrm{F}$

$=F(\mathrm{I}) \mathrm{C}-\mathrm{C})+\mathrm{F}$

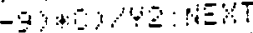

$20=28=(10)$

$-8-1916-42$

$20=8=100$

$=-9=0+2=04$

$-\therefore \cdots=0=0=0=1$

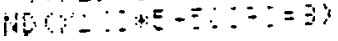

is
$30 \bar{b}=: \vdots-: y=F(50+1)$

$-6 x_{2}=2=-40 F=13 \mathrm{~A}$

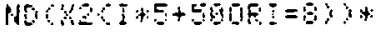

[

390 MEXT

$410 \quad 34=84+85 * 0$

$420=0 \mathrm{I}=4 \mathrm{OTO4Z:F} \mathrm{C}$

$i=F(I) \div F \cap E: 1-35)+1$

EYT

430 FOEI $I=4 T 045: F(I$

$=F(I)+F N C(I-4 Z)+L: H$

EYT

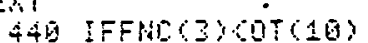

THERE $(47)=F(4)+60 T$ T

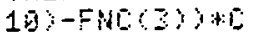

450 IFFME $230 T 11$

THEME $(48)=F(48)+6 \mathrm{FND}$

( $)-0 T(11) ; * 0$

460 IFF=

470 IFYEYF (ES) THENF

$(65)=K E: F(5 E)=0: F(6)$

$\lambda=H$

$480 \quad Y E=0 \quad \therefore \quad \because \cdots$

50 IFO= 1 THENQS9

510 IF $1=01$ THEN' $1=0$

$44=11 \cdot 001099$

$520 \quad 1=0: 15=15+1$

530 FORI $=5 T 0-1: F(I$

$x=F(I Y+F(I-21):$ WEXY

$546 \bar{F}=F(19)+F(1$

j:F(Z1) $=F(21)+F(2): F$

$Q 2)=F(2 Z)+F(3)$

550 FOCI $=1 T 03: F 0$ : 1

$+5)=F(a+17) / F(25$

YC1) : MEYT

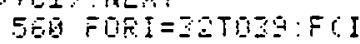

$y=C F C H C S-42)+F(I-$

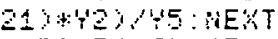

$57=(46)=(F(45))(4)$

$5-12+44 x+5$

806 $I=-1$. IFM=

1099

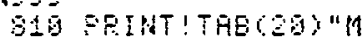

ONTH" "TA "FFINTDUT"

B20 FORI=19TUER : PRI

PIT! "F"I F F I :F(I)

NERT

$320 \quad 14=14: 45=0$

999 I = ER :ENO

999 I = I5R 22$)$; EMO

FFol $\because$ -

TIIIE SEEAIITSE

HEAE B3?

FEINT INTEFUAL

HPS 24 WIVS 9 ?

CH STDFE INTERUAL

HES 5 MINS B?

FUMC STUFE INTERUAL

HES 9 MINS 9 ?

FUNO ZEFO INTERYAL

HES DIMINS 9 ?

$\vdots$ क

CHAMEL DEFAULTS

FMUIOUIGIT!

\# SEZSOR TEFMS 1

COE TOLESS 1

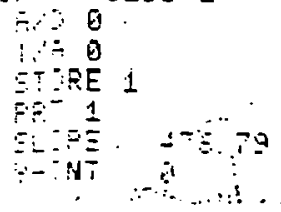

og: $: \because: \vdots: 1$

$12 / 30 / 82-5 / 10 / 83$

90

$\cos$

GTOEE 1

F? 1

SLEE 401.25

Co: Prosess 1

A. 6

$T S 1$

STOEE 1

FRT 1

SLUEE TET.12

$Y-I U T$ - 452.57

COD FFUEESE 1

Fis 0

$T, 1$

STORE 1

PRT 1

ELOFE T75. 16

Y-IHT -455.59

COE PRDEESS 1

A. $\mathrm{C}$ C

$T A 1$

STORE 1

PFT 1

SLOPE TR1.94

$82 / 83$ HEATING SEASON

$T-1 \mathrm{NT}-454.28$

COF PROEESS 1

4,00

$T 1$

STOEE 1

FET 1

ELOFE P74.65

$Y$ INT $\quad-454.01$

COT FEOEES 1

400

$T$ 我 1

STORE 1

FRT 1

SLOFE

$Y$ INT -45245

COH FEOCESS 1

$A \therefore O$

T:F 1

STORE 1 औी

SLRTE 657.13

$Y$-INT - 453.35

COI FROEESS 1

$A, D i$

$T: P$

STOEE 1

FRT 1

SCALE

DFESET B

CEJ FROEESS 1

A.C 6

$T \because A 1$

STOFE 1

FRT 1

SLOFE T3. 309

$Y$ YNT

81. 76

CBK FFOLESS 1

मi, 0

$T, A+1$

ETRE 1

FET I

SLOFE DZ1. IT

ifm I!

$08603-5:$
CDL F $\because=Z \equiv \equiv g$

LOA FEOEES 1 A $C$ C

$T, P 1$

STORE 1

FET 1

ELOFE 2Z0.29

H-IHT . 112

Con FEOSESE 1

A. 1

Tin

FET 1

SCHLE

UFFEET

UFDATE?

STATUS

501 FPT 1

5 GE FPT 1

STI FFT

SEA FET

505 FRT 5

S月O FET

5 ST FET 0

SGE FRT UD

UFEHTE?

OISTFUT

\# DUTFUT TEEHS

UFQATE? 0

$\because$ 
FUNTTION DEFAULTS

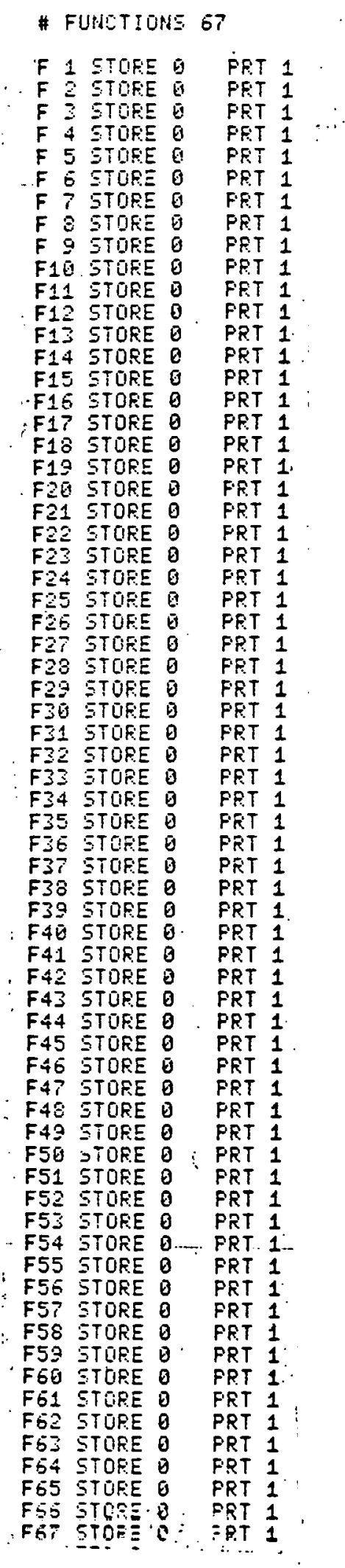




\section{SITE SG}

Mr. \& Mrs. William Moore 85 Robin Hood Road Atlanta, GA 30309

INIT2 Softivare Installed: $11 / 17 / 82$ 


\section{SITE SUMMART SHEET}

Site Identsfication Number SGI

Inst. Contr. Name ESG, Inc.

Address 2231 Perimeter Park

Suite 11 Atlanta, GA 30341

Location:

Town At lanta, GA

Latitude 339.4'

Long 1 =ude $84^{\circ} 3^{\prime}$

Elevation $1005^{\prime}$

Nearest Weather Station:

Atlanta, GA
Building:

Type of Building

General Type of Construction

Ground Coupling

Passive/itybrid syseex(s):

Dowinant Heating System Tyoe

Dominant Heating System Area

Other Systea Type

Othar Syscem Area

Night Insulation.

Auxiliary Heacing System:

syster and Jistribucion iype

Day Tieriostat jetting

Nighe Therzostal Secting

Lirizz zone Floor trez

bassi\% duerzure irea

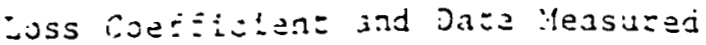

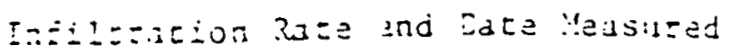

One story residence

Wood frame w/brick veneer

FulT basement
Forced air-natural gas

$\frac{70^{\circ} \mathrm{F}}{70^{\circ} \mathrm{F}}$

$2965 \mathrm{ft}^{2}$

$137.5 \mathrm{ft}^{2}$

$\mathrm{L}=367 \mathrm{Btu} / \mathrm{hr} \mathrm{O}^{\mathrm{F}-1 / 5}$ and $1 / 6 / 83$

No oressure $7 / 3 / 82$ 


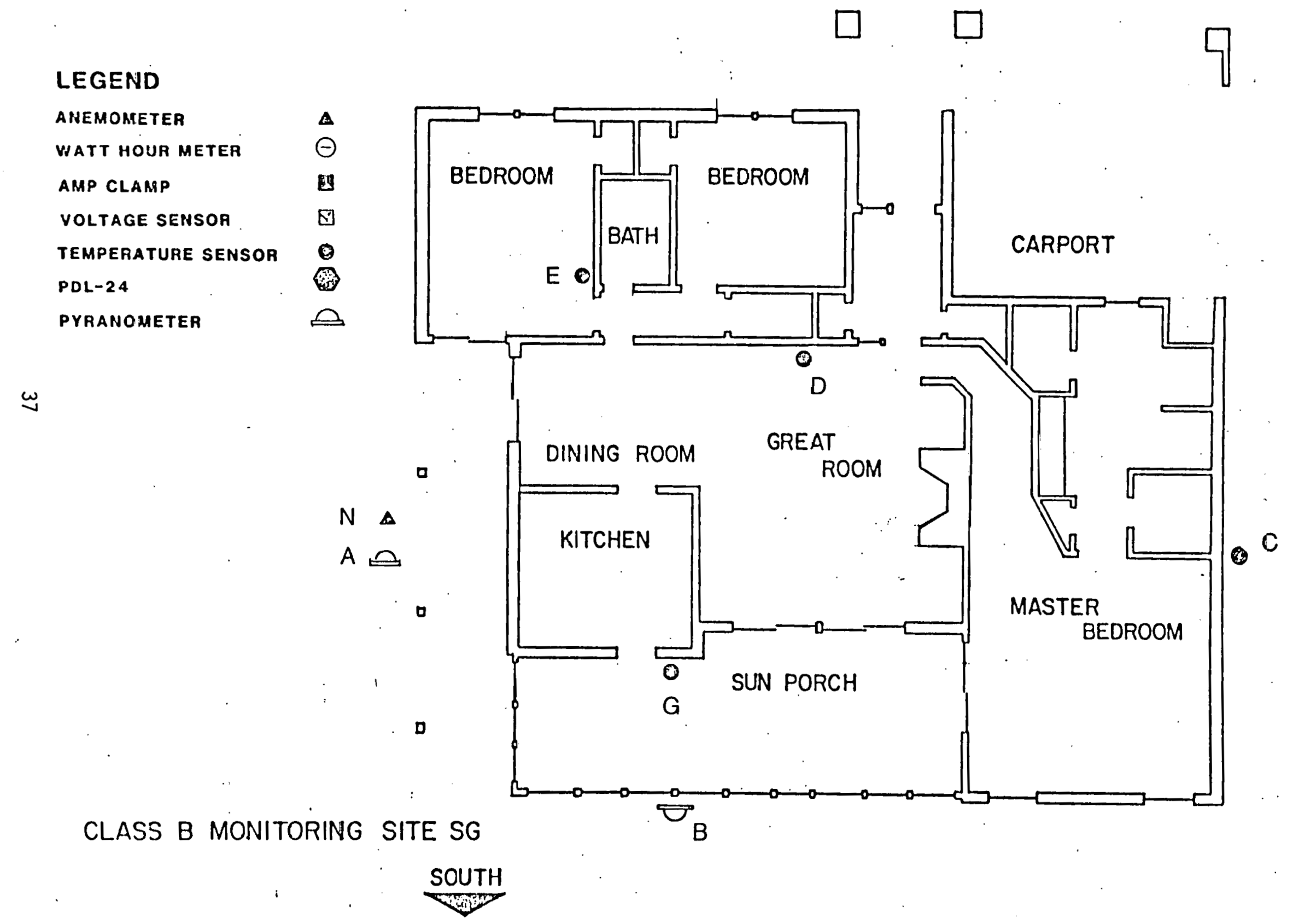




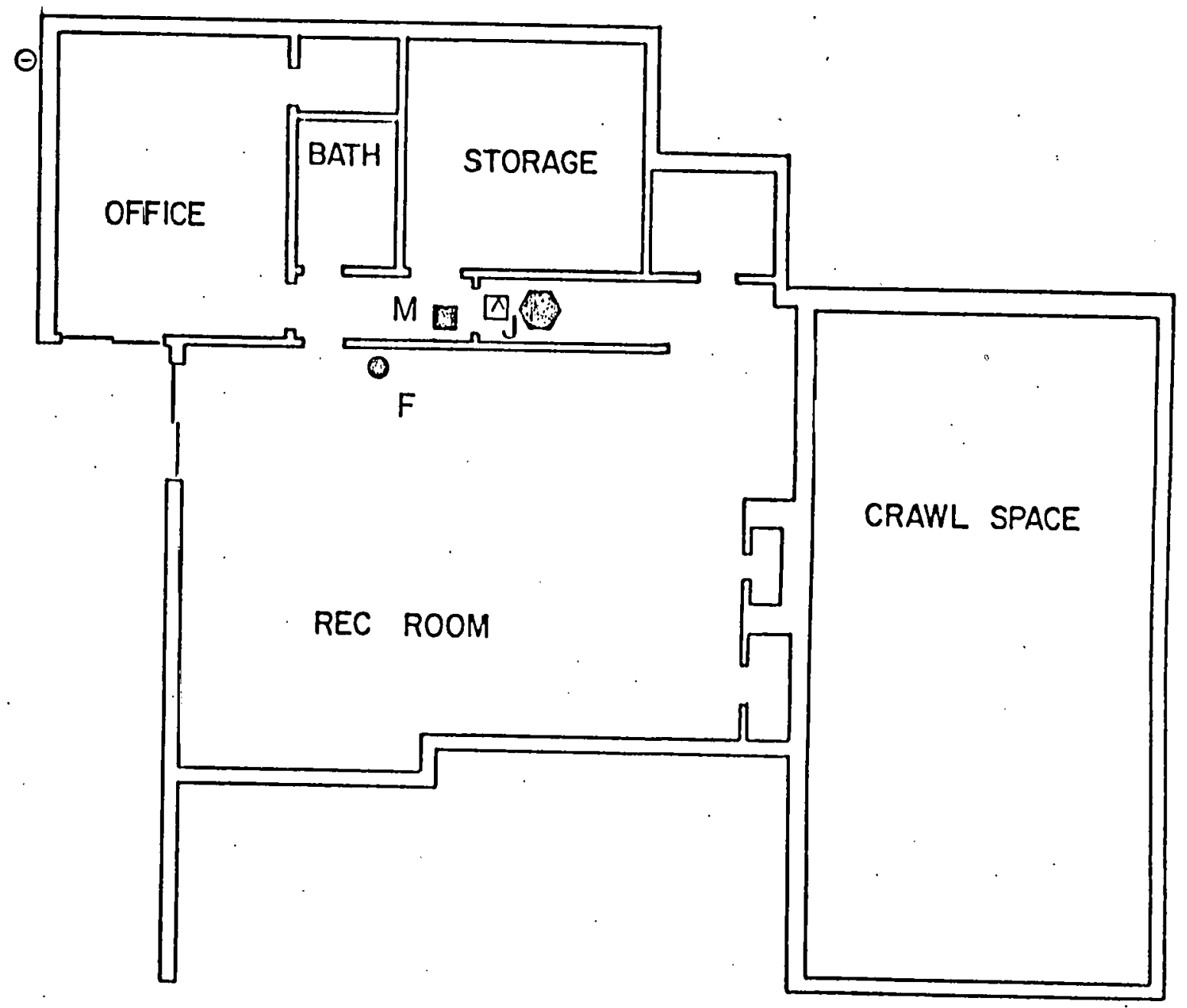

CLASS B MONITORING SITE SG BASEMENT 
Sile Atlanti, Gi

Operator D.H. Gustashaw

One-Tinge Yallege Log

\begin{tabular}{|c|c|c|c|c|c|c|}
\hline $\begin{array}{l}\text { Variable } \\
\text { Name in } \\
\text { Funclions }\end{array}$ & Descripticn & Designation & $\begin{array}{c}\text { Numerical } \\
\text { Value }\end{array}$ & Units & Line \# & Progran \\
\hline OT (1) & $\begin{array}{l}\text { Dverali heat loss } \\
\text { coefficient }\end{array}$ & $\mathrm{L}$ & \begin{tabular}{|l|}
937.8 \\
367.0 \\
\end{tabular} & \begin{tabular}{|l|l|} 
Btu h \\
of \\
\end{tabular} & & $\begin{array}{l}11 / 11 / 82-4 / 8 / 83 \\
4 / 8 / 83-4 / 30 / 83\end{array}$ \\
\hline$O T(2)$ & $\begin{array}{l}\text { Furiace delivery } \\
\text { lifficicncy }\end{array}$ & $n_{f}$ & .60 & - & & \\
\hline OT(3) & Air infiltration rate & I & .75 & $\mathrm{ACH}$ & & \\
\hline OT $(4)$ & $\begin{array}{l}\text { Furnace burner fuel } \\
\text { rlow rate }\end{array}$ & $F_{f}$ & 183 & gal $/ \mathrm{h}$ & & \\
\hline $0 T(5)$ & $\begin{array}{l}\text { Ieating value of } \\
\text { rurnace fuel }\end{array}$ & $\mathrm{HVF}_{f}$ & 1000 & Btu/g & 1 & \\
\hline OT (6) & $\begin{array}{l}\text { later heater burner } \\
\text { ruel flow rate }\end{array}$ & $F_{W}$ & 47 & $\mathrm{gal} / \mathrm{hl}$ & & \\
\hline OT(7) & $\begin{array}{l}\text { leating value of water } \\
\text { Ineater fuel }\end{array}$ & $H V F_{W}$ & 1000 & Btu/g & 1 & \\
\hline$O T(8)$ & $\begin{array}{l}\text { iater heater jacket } \\
\text { leating factor }\end{array}$ & $Q_{w}$ & 500 & Btuh & & $Q_{w}=(.0833)(50)(120-60)(2)=500$ \\
\hline OT $(9)$ & $\begin{array}{l}\text { later neater space } \\
\text { leatirig factor }\end{array}$ & $N_{W}$ & 0.2 & - & & \\
\hline OT (10) & $\begin{array}{l}\text { ux. heat ing set } \\
\text { oint temperatura }\end{array}$ & $\mathrm{T}_{\text {seth }}$ & 70 & ${ }^{o} \mathrm{~F}$ & & \\
\hline OT $(11)$ & $\begin{array}{l}\text { lux. cooling set } \\
\text { loint temperature }\end{array}$ & $T_{\text {setc }}$ & 75 & ${ }^{0} \mathrm{~F}$ & & \\
\hline$O T(12)$ & $\begin{array}{l}\text { :et passive collector } \\
\text { irea }\end{array}$ & $A_{C}$ & $1^{137.5}$ & $\mathrm{ft}^{2}$ & & $\begin{array}{l}11 / 11 / 82-4 / 8 / 83 \\
4 / 8 / 83-4 / 30 / 83\end{array}$ \\
\hline $0 T(13)$ & $\begin{array}{l}\text { loveable insulation } \\
\text { orrection factor }\end{array}$ & $N_{m i}$ & 1 & - & & \\
\hline OT (14) & $\begin{array}{l}\text { lec. aux. heating } \\
\text { oltage mult iplier } k\end{array}$ & $\mathrm{C}_{\text {auxh }}$ & 0 & - & & \\
\hline OT $(15)$ & $\begin{array}{l}\text { lec. water heating } \\
\text { oltage mult iplier } L\end{array}$ & $c_{w}$ & 0 & - & & \\
\hline OT(16) & $\begin{array}{l}\text { hannel M voltage } \\
\text { ultiplier }\end{array}$ & $c_{m}$ & 2 & - & & \\
\hline OT (17) & $\begin{array}{l}\text { hannel if voltage } \\
\text { witiolier }\end{array}$ & $c_{n}$ & 0 & - & & ' \\
\hline
\end{tabular}




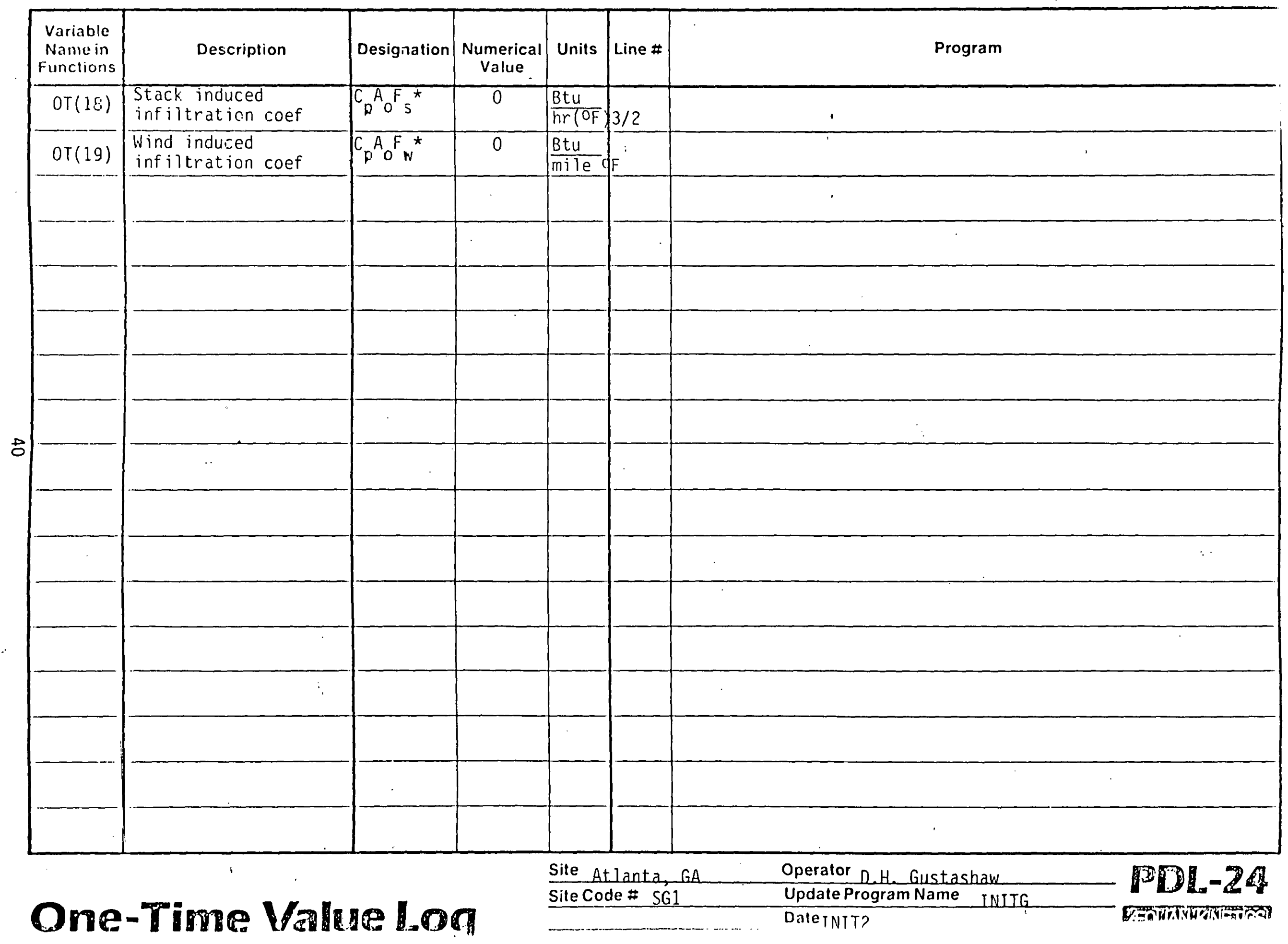




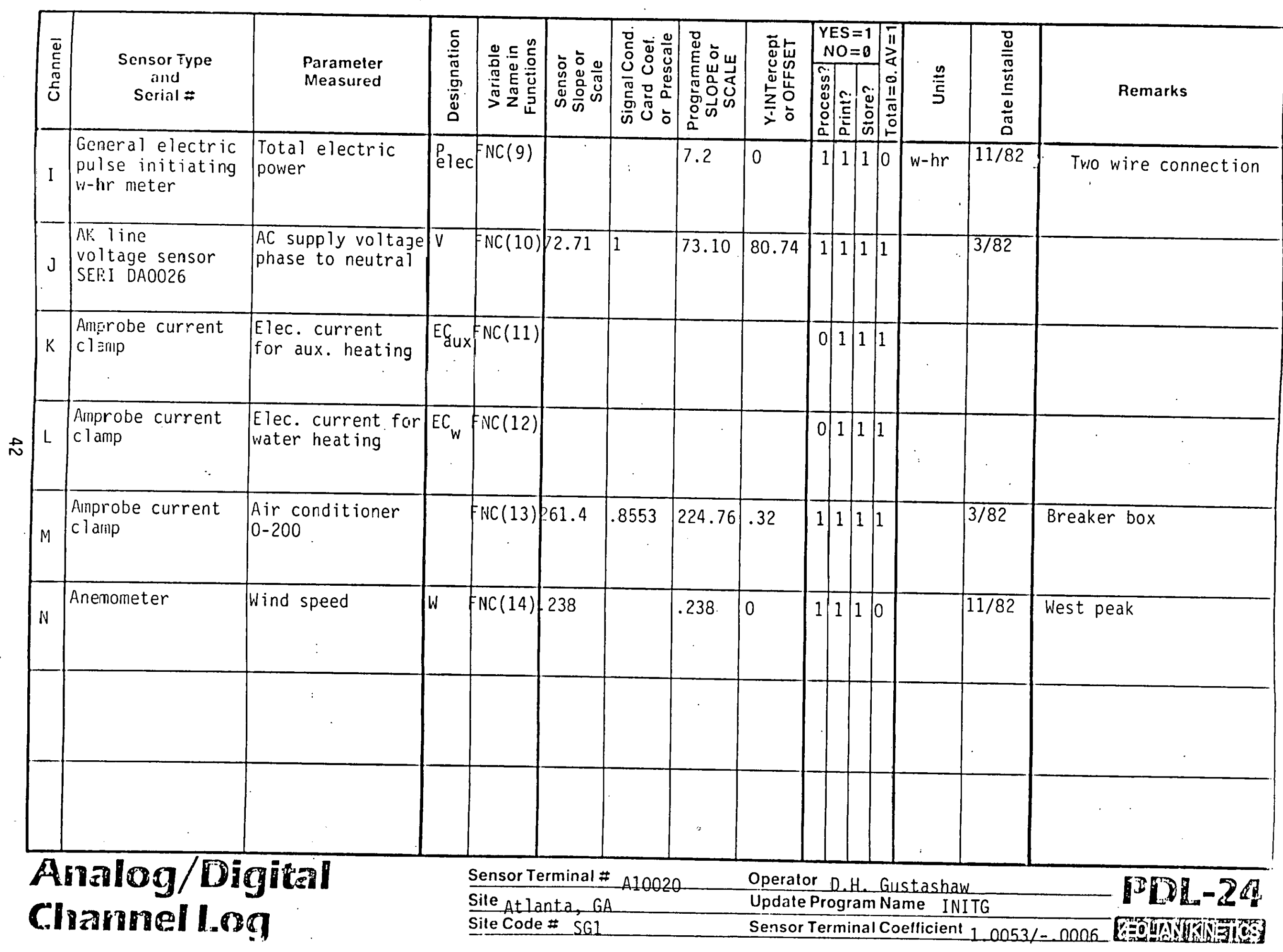




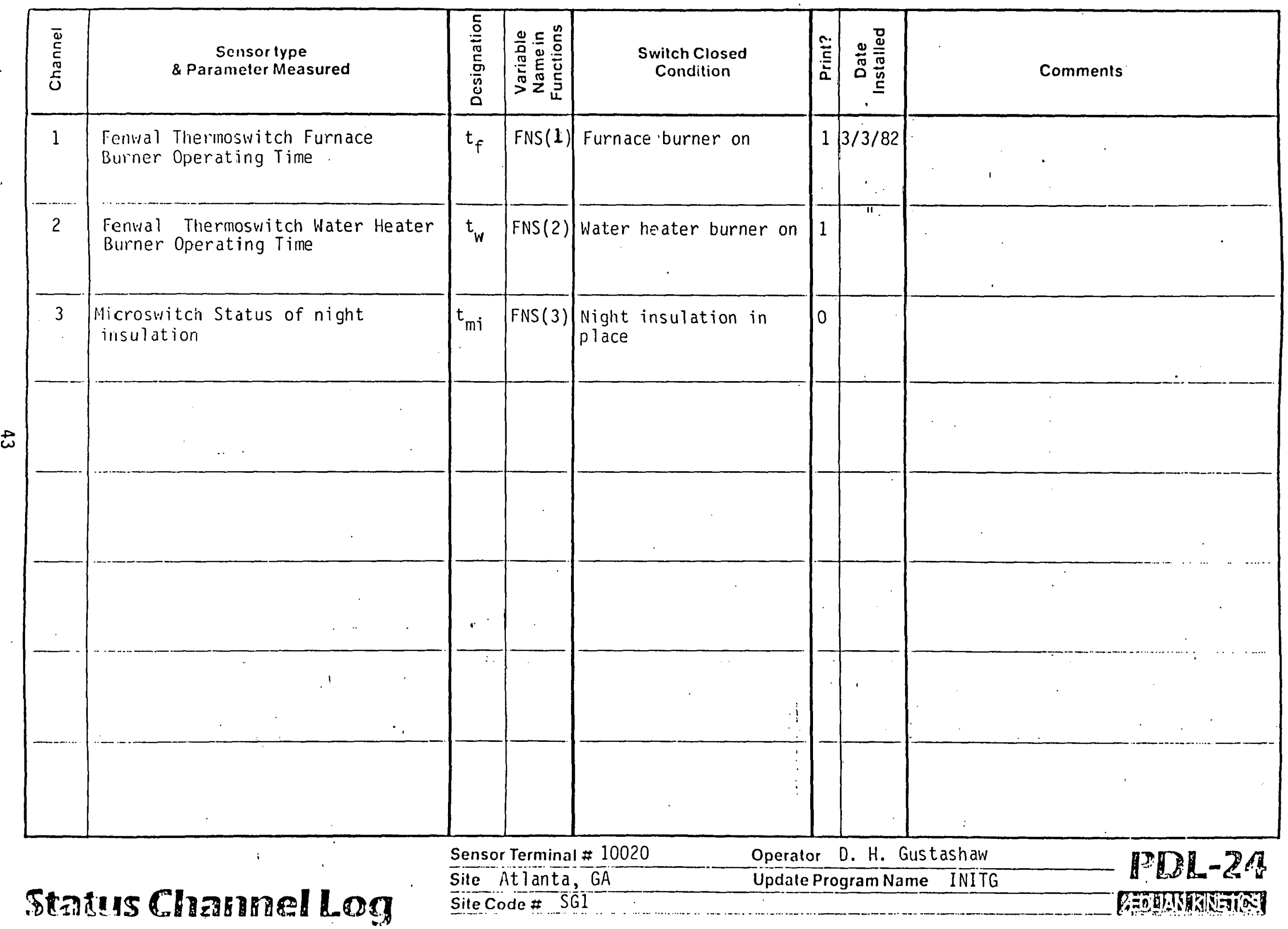


INIT $G$.

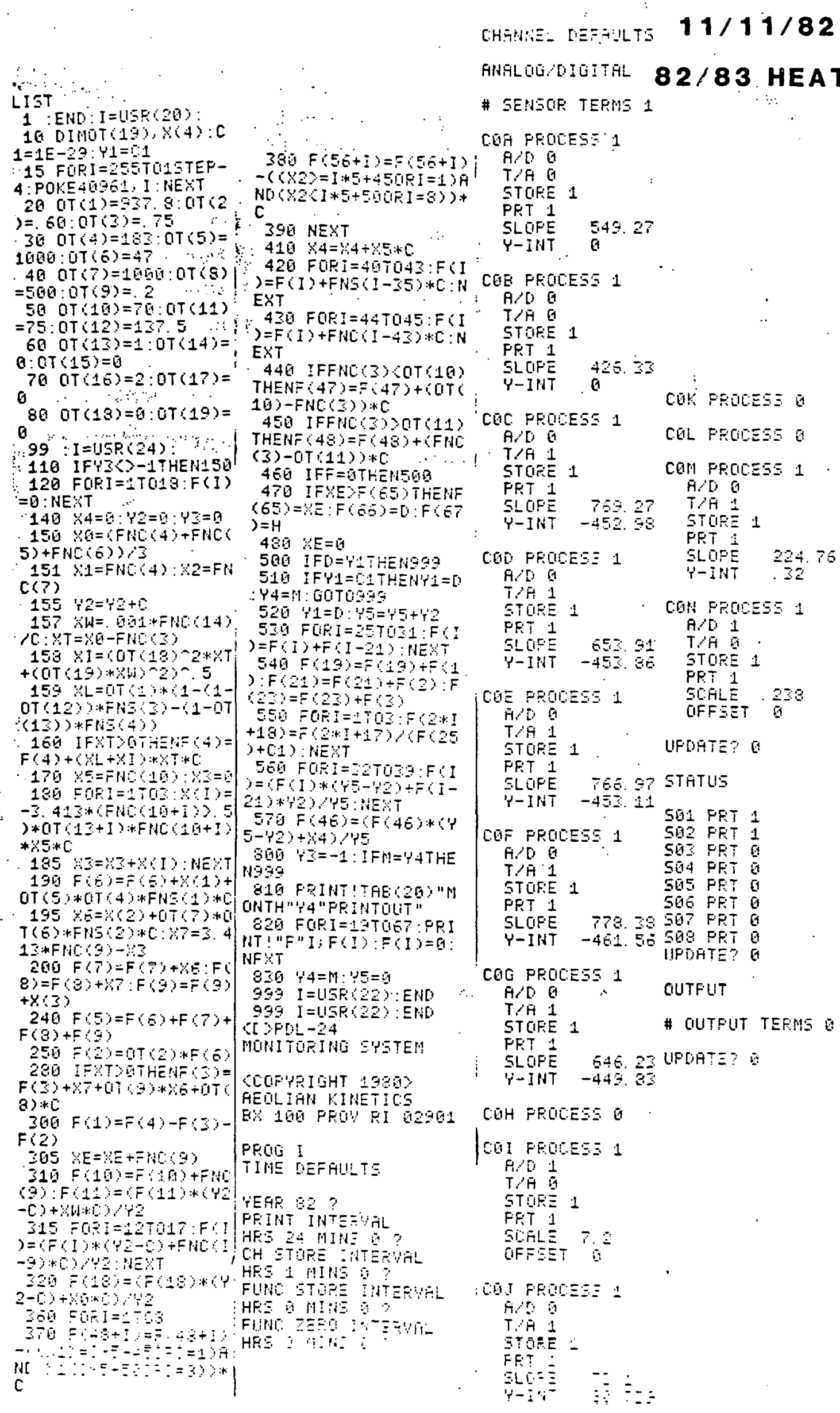


FOAETION DEFEISTE

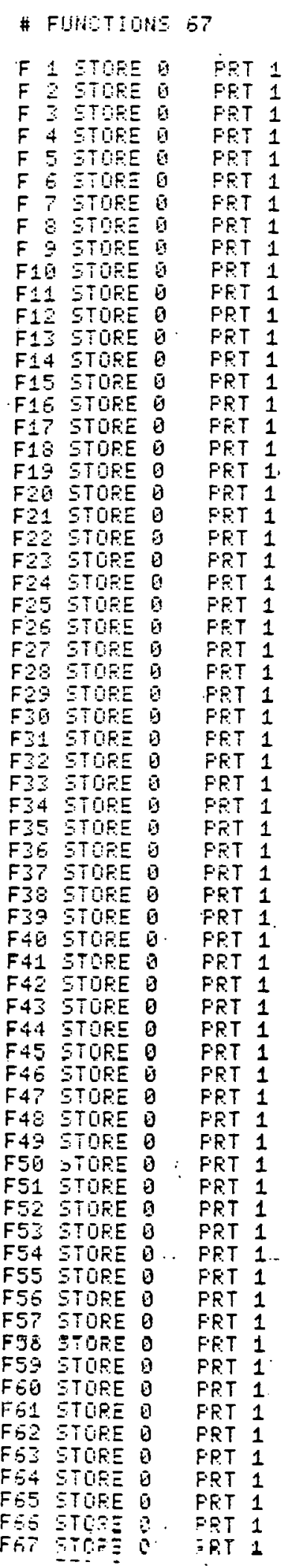


$360=:=2-i=F(56+1)$

$-(62)=1+5+45):=4) A$

MOC.6\%

LIST ! $\because$

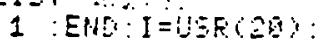

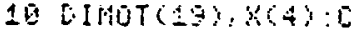
$1=\angle E-2:\langle: 1=1$ 15 FOFI IOSTOS STEF-

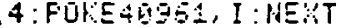

- 20 OT (1)=367:OT(2)= EB:OT $(3)=75$

30 OT $(4)=183: 0 T(5)=$ $1000: 0 T(E)=47$

$\therefore 40$ OT $(7)=1000: 0 T(s)$

$=500: 0 T(9)=$

- 50 OT(10:=70:0T(11)

$=75:$ UT $(12)=1 \cdot \overline{0}$ 0: OT $(15)=0$

70 OT $(16)=2: 0 T(17)=$

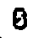
80 OT $(13)=0: 0 T(19)=$

0

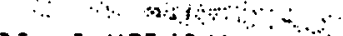
$99: I=135 F(24)$.

11D IFY SO-1THEN150 120 FORI $=1 T 018: F(I)$

$:=0:$ NEXT $: T$

$\because 140 \times 4=0: 42=03: 43=0$

$150 \quad \angle \bar{O}=C$ CFHC $(4)+F H C C$

$5)+F M(5) ; 3$

$151: \because 1=F N C: 4:: 2=F M$

C ( 7 )

$155 \% 2=12+1$

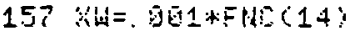

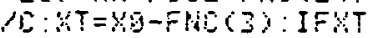
<可

$158 \% !=60 T(18)-2 \% \%$ + (OT 613 ) $\left.x_{4}\right)=2$ ) 5

159 $\mathrm{SL}=0 \mathrm{~T}$ (A)*C1-C1UT (12) WFHECO-(1-OT

(13) +FNEC4)

1ED IFXT UTHENF 4 ) $=$

$F(4)+C(L+X)+x T+C$

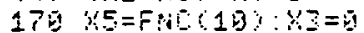

120 FIFI = 1 TOS: $3:=$

-3. $432+(F N E C 10+53.5$

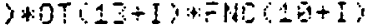

*:5 5 +

$185 \quad x=Y 3+Y C I:$ HEXT

$19 \bar{F} \overline{C E})=F(6)+3(1)+$

OT $(5)$ \%TC4 *FNEC1)*L

$195: 5=2(2)+1 T(7) * 0$

T(E)*FHEC 2$) * C: M 7=3.4$

$13+F 609)-3$

200 $F C P=F(B)+\{E: F C$

$s)=F(\theta)+37: F(9)=F(9)$

$+2(3)$

$245 F(5)=F(6)+F(7)+$

$F(\overline{6})+56)$

$250 F 2) 0 T(2)+F(E)$

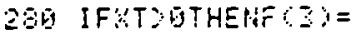

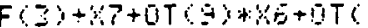

8) $:+: 10$

उण̄ $F(1)=54)=(3)-$

F(2)

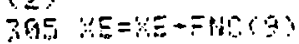

$310=(10)=F(15)+F N$

$\left.(9) F(11)=F^{2}(11)+C^{\prime} T^{\prime}\right)$

-0 16: 12

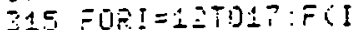

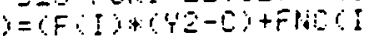

$-9+21 \%$ 1

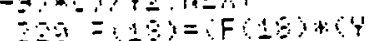

$2-20000$

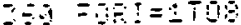

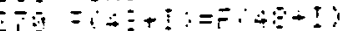

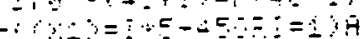

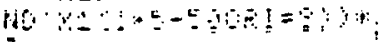

i
C

390 HEXT

$416 \quad \times 4=44+35+5$

420 POSI $=407043: F C I$

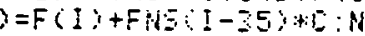

EXT

$43 B=D F I=4-T O 4.5: F C I$

$\lambda=F(I)+F N O C I-43)+B: H$

EXT

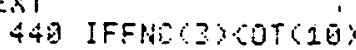

THEHF $(47)=F(47)+C O T C$

10)-FMCOSI+C

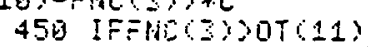

THEN $(48)=F(48)+C F M C$

( 3$)-0 T(11)$ )

460 IFF=日T THE

47 IFUES CESTHENF

$(55)=X E: F E S)=0: F \& E$

$\mathrm{r}=\mathrm{H}$

$430: \because E=\overline{0}$

500 IFO= 1 THEMPS9

519 IF' $1=6$ THEU' $1=0$

Y $4=14: 00 T 0993$

520 - $1=0: 15=45+12$

5SE FOEI=2STUS1:FCI

)$=F(I)+F(I-21): N E: T$

$540 F(1)=619)+F(1$

$\therefore F(21)=F(2)+F(2): F$

(2Z)=F(2Z)+F 2$\}$

$550=05=1 T 03: F 921$

$+12)=F(2 * I+17)$ C ( F $(25)$

$i+C 4:$ NEKT

560 FOEI $=37029 \div C I$

$i=C F(I) *(45-42)+F(I-$

21)* 145 :

$570 F(40)=F(4 E)+C$

$5-42)+44)$

800 YZ $=-1$ :IFM= 4 THE 1159

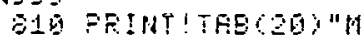

DRTH:"A "FRIATOUT"

O2Q FUFI = OTOET:FFI

MT! "T "I F F

MEXT

$8 \geq 5$ : $4=r_{1}: t^{\prime}=5$

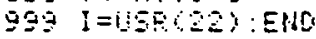

G95 I=USF $22:$ :ENO

it $>\mathrm{FCL}-24$

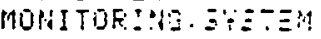

FFín I

TIME GEFFULTS.

YFAF $Q 2 ? \mathrm{Q}$

FFINT INTEFUTL

HFS 24 MI ISE

$\mathrm{CH}$ STUEE IMTEPUAL

HES 1 MIMS 9 ?

FUNO STOFE INTERUAL

HFE 0 Y I ISE

FUMUL EETO INTEFUHL

HES 0 MINSO

CHANHEL DEFAILTE

RHALOBYOBTTAL

\# SEMEGE TEZHS 1

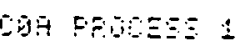

H. $\mathrm{T}:$

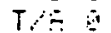

FF: :

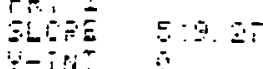

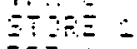

INIT G

$4 / 8 / 83-5 / 6 / 83$

CQS FOZ:S1

A. 0 a

$T, A \quad 6$

STIJES 1

FRT 1

SLOFE

Y-INT

425.23

CDC FFOCES 1

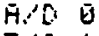

TP 1

STOFE 1

PFT 1

BLOEE 78027

Y-IVT - 45.98

CBD FEOCES: 1

A.O 0

$T, \hat{H}$ i

STORE 1

FRT 1

SLOFE 653.91

T-IMT -453.86

CDE FFOCES: 1

Ha 00

$T \therefore A$

STOEE 1

FRT A

SLOFE TEE T7

$Y-I \& T \quad-45 Z 11$

COF FEOEDSE 1

4,0

$T \cdot T 1$

STOEE 1

FRT I

GLUFE TTO.

7 -IHT -481.50

COS FFOEES 1

Aid 0

$T \because \bar{H} 1$

STOEE 1

FET 1

SLOFE 84023

$Y$ INT -449.83

COH FROCES:

CQI FRODESS 1

$A: D 1$

$T, \bar{G}$

STCFE 1

F.FT 1

SCPLE 7.2

UFFEE 9

CDI FFOE:SI 1

A. 6

$T$

GTOEE 1

FET 1

Elge

T-INT 8.73

CQY FEDEE

C년 Fon

STORE 1

FCATE?
$\$ 2 / 83$ HEATING SEASON

Din $F=51$

Fis

T. 1

FET 1

SLFE 24.75

on feocess 1

Fid 1

$T$ A 0

STORE 1

FRT 1

BLE . 230

FOATE?

TATUS

DI FET

$02 \mathrm{FET}$

ge est

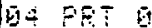

DE FET

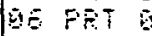

G F Fi

Q98 FET

UTFUT

OUTEUT TEEHS

FDATE? 


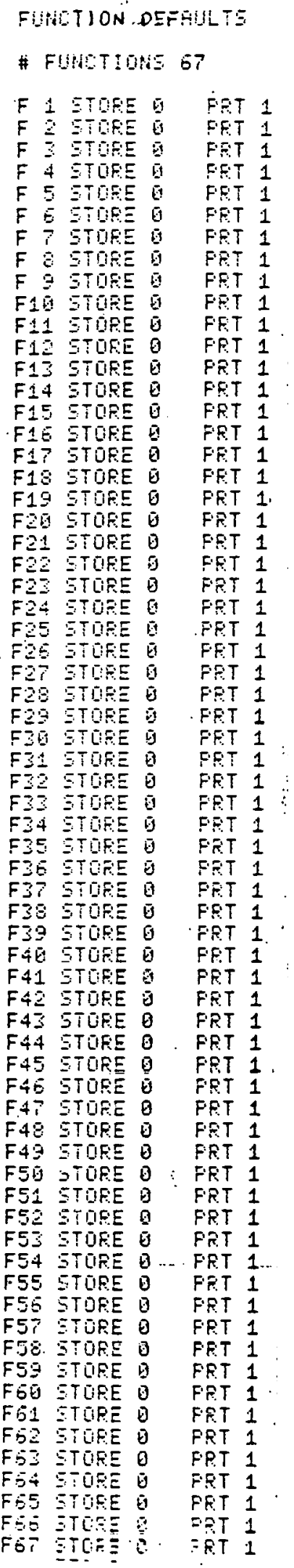


SITE SJ

Mr. \& Mrs. Jack Cook

P.0. Box 449

Manning, SC 29102

INIT2 Softivare Installed: 11/15/82 
Site Identfication Number Sul
Inst. Contr. Nane ESG, Inc.

Address 2231 Perimeter Park

Suite 11 At]anta. GA 30341

Location:

Town Manning. S.C.

Lat1tude $3400^{\prime}$

Longi=ude $80^{\circ} 3^{\prime}$

Elevation 291.'

Nearest weather Station:

Sumter-ShaidAEB

Building:

Type of Building

General Type of Construction

Ground Coupling

One story residence

wood frame and bric's veneer

cravlspace

Passive/Hybrid syscez(s):

Dowinant leating System Type

Dominant tiez=ing Systed Area

Other Systea Tipe

Other syscez irez

Nizhe Insuiation

Auxiliary Yeating System:

Jystea and Jiscrioucion Type

Day Thersostat jetting

Migne Therzostat Setting

Lizinz Zune Fuor irez

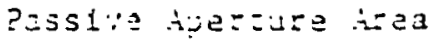

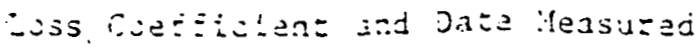

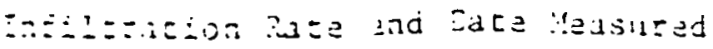

Sunspace

$191 \mathrm{ft}^{2}$

Trombe wall

$200 \mathrm{ft}^{2}$

$N / A$

Electric forced air

\begin{tabular}{|c|}
\hline $68^{\circ} \mathrm{F}$ \\
\hline $68^{\circ} \mathrm{F}$ \\
\hline 2755 Et 2 \\
\hline $301 \mathrm{ft}$ \\
\hline$L=3252$ Etw $3 / 2$ and $3 / 3 / 53$ \\
\hline No aressure $8 / 9 / 8 ?$ \\
\hline
\end{tabular}




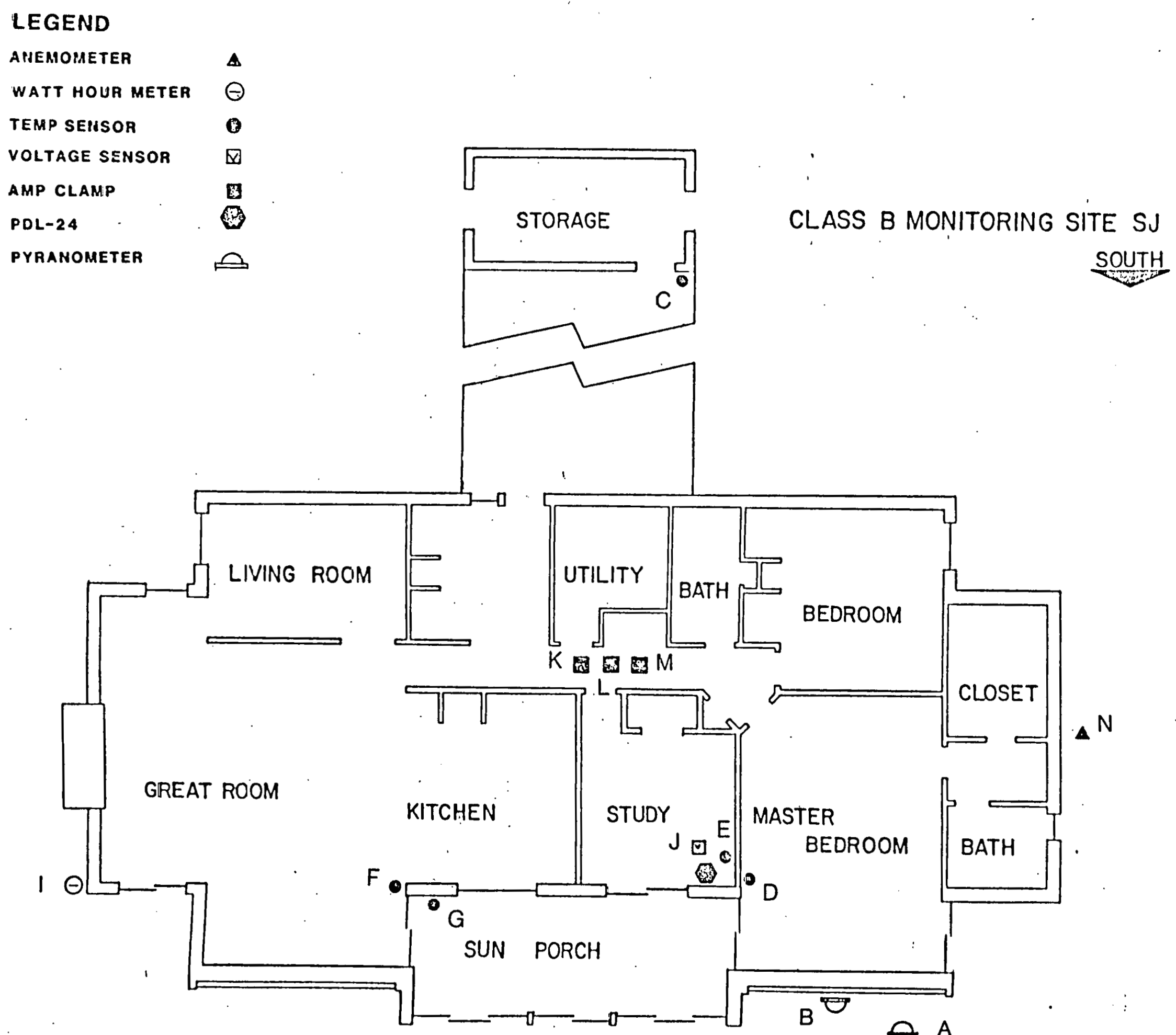




\section{Orbe-Tine Valug log}

Sile Manning,S.C. Operator Q.H. Gustashaw Site Code F U.11 Update Program Name INITJ

\begin{tabular}{|c|c|c|c|c|c|c|}
\hline $\begin{array}{l}\text { Virriable } \\
\text { Name in } \\
\text { Functions }\end{array}$ & Description & Designation & $\begin{array}{c}\text { Numerical } \\
\text { Value }\end{array}$ & Units & Line $\#$ & Program \\
\hline or $(1)$ & $\begin{array}{l}\text { Overall heat loss } \\
\text { coefficient }\end{array}$ & $\mathrm{L}$ & $\begin{array}{l}827 \\
325.2\end{array}$ & \begin{tabular}{|l|l|}
$\mathrm{Btuh}$ \\
${ }_{\mathrm{O}}$ \\
\end{tabular} & & $\begin{array}{l}11 / 15 / 82-2 / 28 / 83^{1} \\
3 / 3 / 83-4 / 30 / 83 \\
\end{array}$ \\
\hline OT (2) & $\begin{array}{l}\text { Furnace delivery } \\
\text { ufficiensy }\end{array}$ & $n_{f}$ & .95 & - & & \\
\hline $\operatorname{OT}(3)$ & Air infiltration rate & $I$ & .75 & $\overline{\mathrm{ACH}}$ & & \\
\hline $\operatorname{OT}(4)$ & $\begin{array}{l}\text { Furnace burner fuel } \\
\text { flow rate }\end{array}$ & $F_{f}$ & 0 & gal/hr & & \\
\hline OT $(5)$ & $\begin{array}{l}\text { lleating val te of } \\
\text { furnafe fuel }\end{array}$ & HVF $_{f}$ & 0 & Btu/ga & & \\
\hline OT $(6)$ & $\begin{array}{l}\text { Water heater burner } \\
\text { fuel flow rate }\end{array}$ & $Q_{W}$ & 0 & gal/hr & & \\
\hline $0 T(7)$ & $\begin{array}{l}\text { lieating value of water } \\
\text { heater fuel }\end{array}$ & $H_{W}$ & 0 & $B t u / g a$ & & \\
\hline OT $(8)$ & $\begin{array}{l}\text { Water heater jacket } \\
\text { heater factor }\end{array}$ & $Q_{w}$ & 0 & Btuh & & Water heater in attic \\
\hline OT(9) & $\begin{array}{l}\text { Water heater space } \\
\text { lieating factor }\end{array}$ & $N_{w}$ & .1 & - & & \\
\hline $0 \mathrm{~T}(10)$ & $\begin{array}{l}\text { Aux. heating set } \\
\text { point temperature }\end{array}$ & Tsetr. & 68 & ${ }^{\circ} F$ & & \\
\hline $0 T(11)$ & $\begin{array}{l}\text { Aux. cooling set } \\
\text { point temperature }\end{array}$ & $T_{\text {setc }}$ & 75 & ${ }^{\circ} \mathrm{F}$ & & . \\
\hline OT (12) & $\begin{array}{l}\text { llet passive collector } \\
\text { area }\end{array}$ & $A_{C}$ & 391 & $\mathrm{ft}^{2}$ & & \\
\hline $\operatorname{or}(13 i$ & $\begin{array}{l}\text { Hoveable insulation } \\
\text { correction factor }\end{array}$ & $\mathrm{N}_{\mathrm{mi}}$ & 1 & - & & \\
\hline OT $(14)$ & $\begin{array}{l}\text { Elcc. aux. heating } \\
\text { voltage multiplier }\end{array}$ & $C_{\text {auxh }}$ & 2 & - & & \\
\hline OT(15) & $\begin{array}{l}\text { Elec. Water neating } \\
\text { voltage multiplier }\end{array}$ & $c_{w}$ & 2 & - & & \\
\hline OT $(16)$ & $\begin{array}{l}\text { Channel in voltage } \\
\text { inultiplier }\end{array}$ & $\bar{c}_{m}$ & 2 & - & & \\
\hline oT $(17)$ & $\begin{array}{l}\text { Channel if voltage } \\
\text { uwltioliar }\end{array}$ & $c_{n}$ & 0 & - & & \\
\hline
\end{tabular}




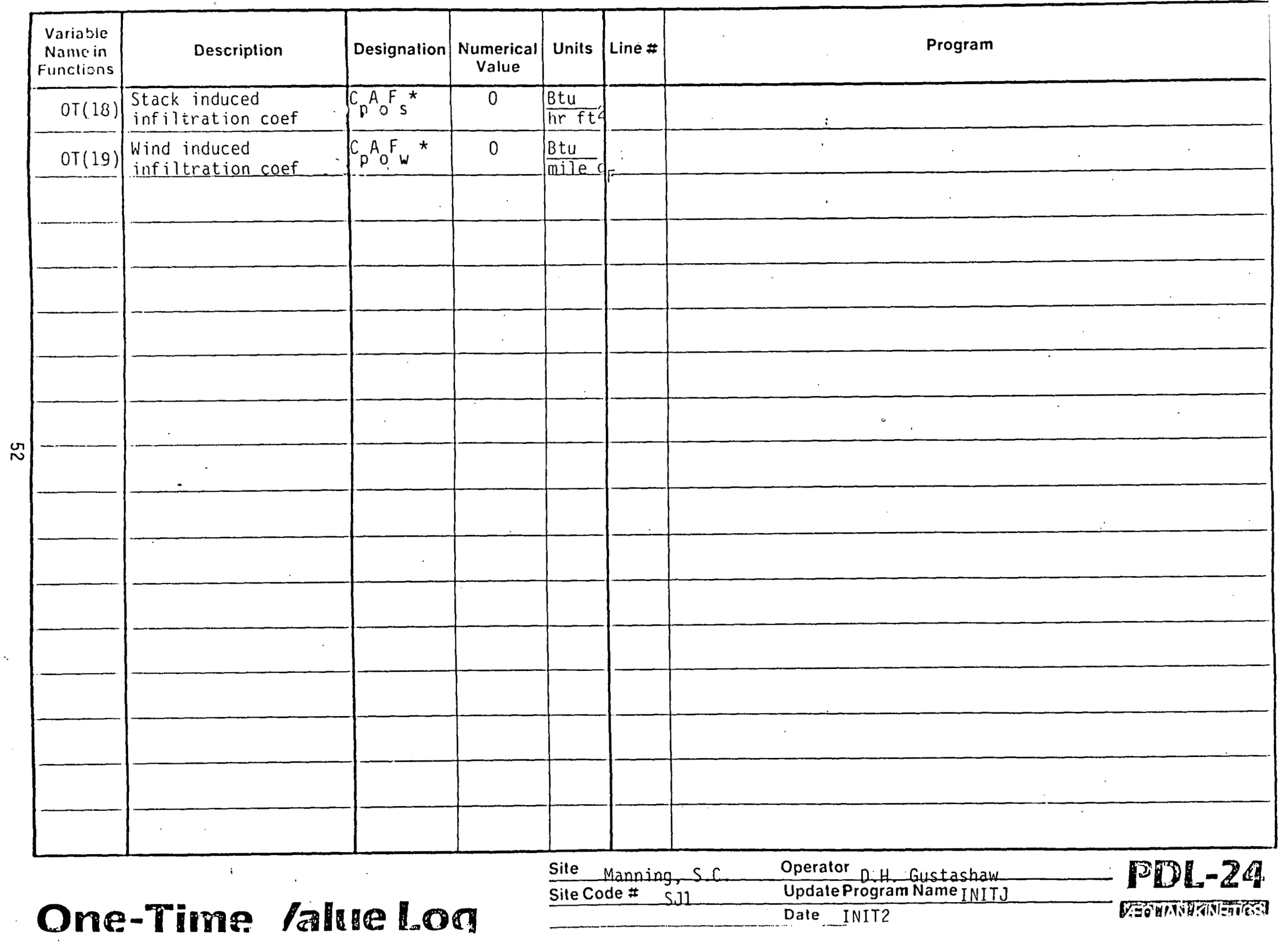


Al6

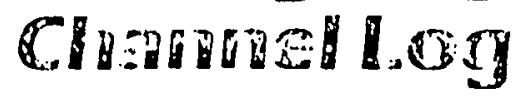

Sensor Terminal $\#$ A 10124

Sile Manning. S.C

Sile Code \# S.l1.
Operalor D.H. Gustashaw Updale Program Name INITI Sensor Terminal Coelficient 1.0048
1010-24

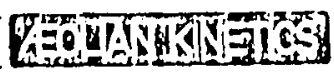

\begin{tabular}{|c|c|c|c|c|c|c|c|c|c|c|c|c|c|c|c|}
\hline \multirow{2}{*}{$\begin{array}{l}\vec{a} \\
\stackrel{c}{c} \\
0 \\
\tilde{\sigma} \\
\mathcal{U}\end{array}$} & \multirow{2}{*}{$\begin{array}{c}\text { Sensor Type } \\
\text { and } \\
\text { Serial }=\end{array}$} & \multirow{2}{*}{$\begin{array}{l}\text { Parameler } \\
\text { Measured }\end{array}$} & \multirow{2}{*}{$\begin{array}{l}\frac{5}{0} \\
\frac{0}{0} \\
\frac{0}{n} \\
0 \\
0\end{array}$} & \multirow{2}{*}{ 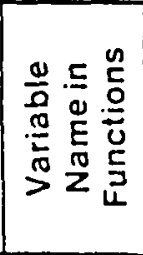 } & \multirow{2}{*}{ 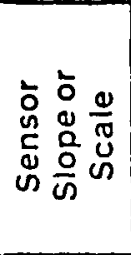 } & \multirow{2}{*}{ 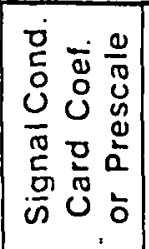 } & \multirow{2}{*}{ 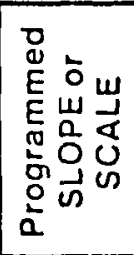 } & \multirow{2}{*}{ 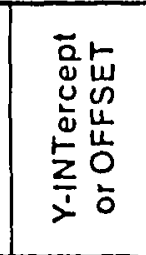 } & \multicolumn{3}{|c|}{$\begin{array}{l}\text { YES }=1 \\
N O=0\end{array}$} & \multirow{2}{*}{$\begin{array}{c}7 \\
11 \\
2 \\
0 \\
0 \\
11 \\
\frac{2}{0} \\
0 \\
1\end{array}$} & \multirow[b]{2}{*}{$\stackrel{n}{5}$} & \multirow{2}{*}{$\begin{array}{l}\frac{D}{0} \\
\frac{0}{0} \\
\frac{n}{5} \\
\frac{0}{\infty} \\
0\end{array}$} & \multirow[b]{2}{*}{ Remarks } \\
\hline & & & & & & & & & 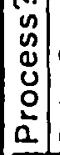 & $\mid$ & $\left|\begin{array}{c}c \\
\dot{\alpha} \\
\frac{\alpha}{n}\end{array}\right|$ & & & & \\
\hline$A$ & $\left\{\begin{array}{l}1 \text { iCom pylanometer } \\
\text { IY } 2730 \\
\text { SERI AAO127 }\end{array}\right.$ & $\begin{array}{l}\text { Incident solar } \\
\text { radiation on the } \\
\text { rorizontal }\end{array}$ & $I_{h}$ & $F N C(1)$ & $\begin{array}{l}4.0025 \\
\times 10^{6}\end{array}$ & 9959 & 403.83 & 0 & 1 & 1 & 1 & 0 & & $12 / 81$ & Center of roof \\
\hline$B$ & $\begin{array}{l}\text { I. iCor pyranomater } \\
\text { PY } 2731 \\
\text { SERI AA0128 }\end{array}$ & $\begin{array}{l}\text { Incident solar } \\
\text { radiation on } \\
\text { collector plane }\end{array}$ & $I_{\text {in }}$ & $F N C(2)$ & $\begin{array}{l}5.6912 \\
\times 10^{6}\end{array}$ & 9971 & 573.52 & 0 & 11 & 1 & 1 & 0 & & 11 & $\begin{array}{l}\text { Center of trombe } \\
\text { wall }\end{array}$ \\
\hline$C$ & $105017 \mathrm{~J}$ & $\begin{array}{l}\text { putdoor dry } \\
\text { pulb temperature }\end{array}$ & $T_{0}$ & FNC (3) & $\begin{array}{l}1.7847 \\
\times 10^{6}\end{array}$ & 320.5 & 772.79 & -458.51 & 11 & 1 & 1 & 1 & & 11 & West side of house \\
\hline 0 & id $590 \mathrm{0}$ & $\begin{array}{l}\text { Inside dry bulb } \\
\text { temperature }\end{array}$ & $T_{i}$ & FNC (4) & $\begin{array}{l}1.7821 \\
\times 10^{6}\end{array}$ & 2731.06 & 554.98 & -455.7 & 11 & 1 & 1 & 1 & & 11 & $\begin{array}{l}\text { Master bedroom } \\
4^{1}-7 \frac{1}{2}{ }^{\prime \prime} \text { from floor }\end{array}$ \\
\hline$E$ & (iD 590J & $\begin{array}{l}\text { nside dry bulb } \\
\text { emperature }\end{array}$ & $T_{\mathbf{i}}$ & FNC (5) & $\begin{array}{l}1.7932 \\
\times 10^{6}\end{array}$ & 2760.25 & 552.08 & -458.7 & 11 & 1 & 1 & 1 & & 11 & $\begin{array}{l}\text { Hobby room } \\
5^{\prime}-0^{\prime \prime} \text { from floor }\end{array}$ \\
\hline $\mathrm{F}$ & $\therefore 5900$ & $\begin{array}{l}\text { Inside dry bulb } \\
\text { emperature }\end{array}$ & $T_{i}$ & FNC (6) & $\begin{array}{l}1.7868 \\
\times 10^{6}\end{array}$ & 2735.26 & 655.70 & $-457.8\}$ & 1 & 1 & 1 & 1 & & 11 & Living room \\
\hline$G$ & 10590. & $\begin{array}{l}\text { Eunspace dry } \\
\text { Eulb temperature }\end{array}$ & $T_{s}$ & $F N^{\prime} C(7)$ & $\begin{array}{l}1.7592 \\
\times 10^{6}\end{array}$ & 2728.9 & 547.05 & -448.7 & 11 & 1 & 1 & 1 & & 11 & \\
\hline$H$ & ii 590. & & & FNC(8) & & & & & 0 & 1 & 1 & 1 & & 11 & \\
\hline & & . & & & & & & & & & & & & & \\
\hline
\end{tabular}




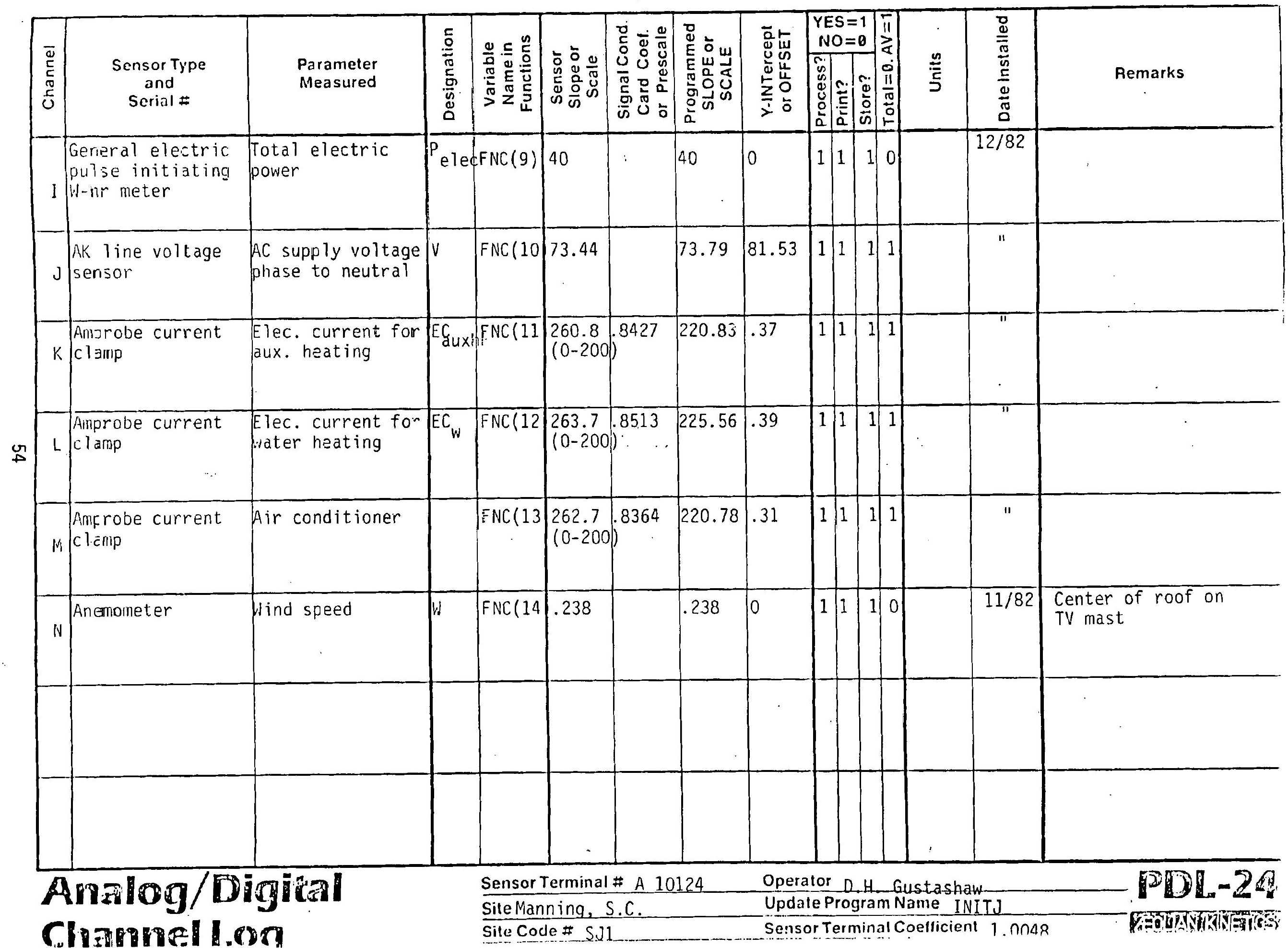




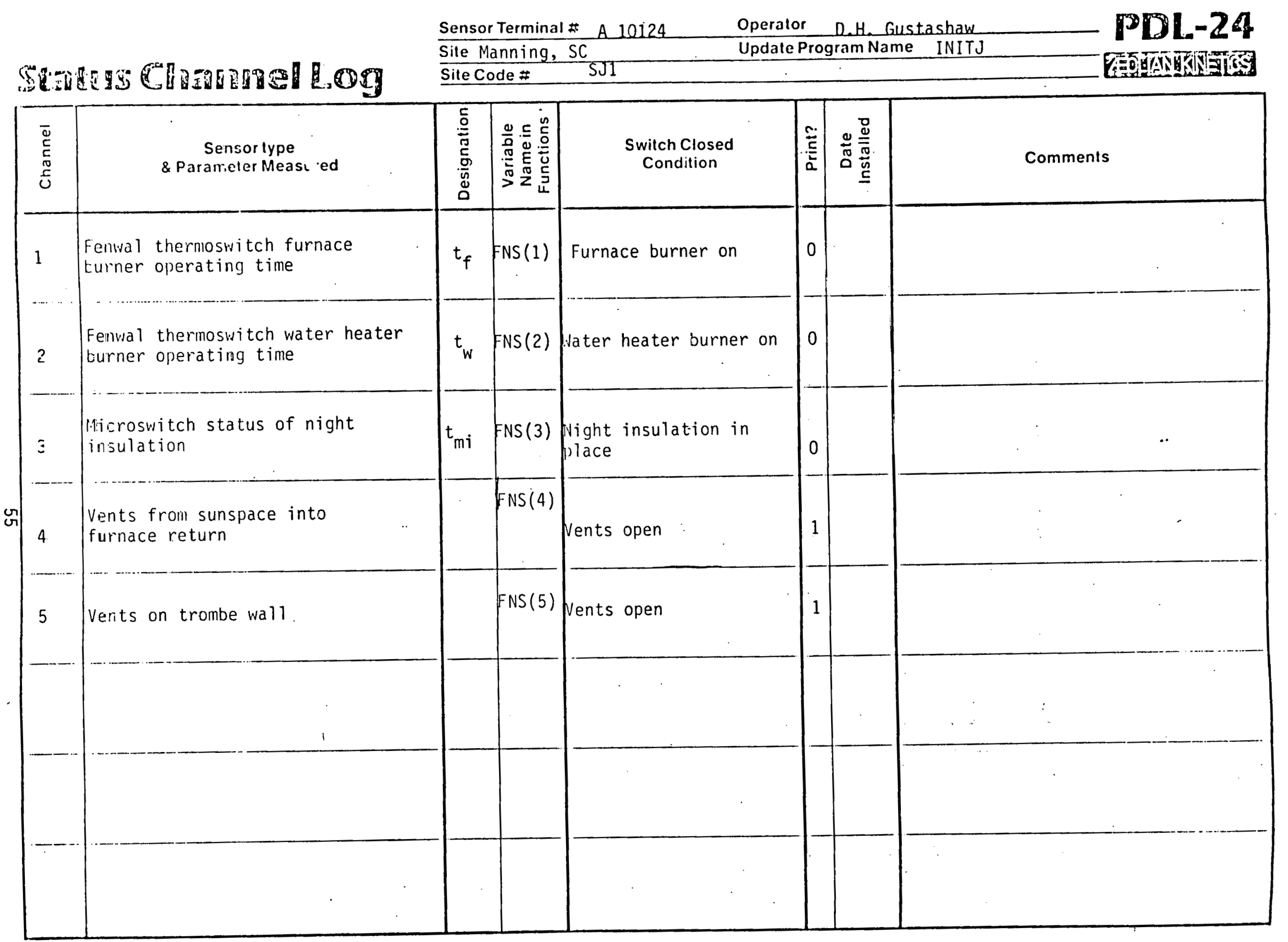




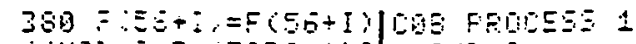

Lisi

1 :ENO: 1=JSF(EA: $\quad 393$ KEXT

10 D!MOT(19), K(4):L $1= \pm E-2=Y^{\prime} 1=01$

15 FIR! = 25STDASTEF4:FOKE4BSS1, I :NEYT

20 OT $(1)=527: 0 T(2)=$ $\therefore 95: 0 T(3)=75$

30 OT $(4)=0:$ OT $(5)=5$ OT $(6)=0$

IO OT $(2)=0$ OT $(\varepsilon)=0$

OT $(9)=1, \because \cdots, \cdots$

$\because 50$ OT 110$)=58: 0 T$ (11)

$=75: 0 T(12)=39$

60 OT $(13)=1: \operatorname{OT}(14)=$ 2:OT $(15)=2$

:70 OT $(15)=2:$ OT $(17)=$

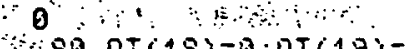

Ho OT $(18)=39:$ OT $(19)=$

O 09 I $=U 5 F(24)$

H 110 IFY3E-1THEH15日

120 FURI $=1$ TO1E:F(I)

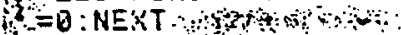

$4: 140 \quad \times 4=0: 4:=0: Y^{\prime} 3=0$

15B $Y U=C$ FHIC $(4)+F H C C$

-5) $+F P(6)) 3$

$151: 31=F N E(4): K 2=F N$

C(7)

$155 \% 2=12+c$

$157 \therefore W=$. BDI $*$ FHEC14: IC: $: T=Y M-F H E(S): I F X T$

CQTHEVXT=D

$15 \%$ : I = COT 12$)^{\circ} 24 \% T$

+coT (19)*wW:-2). 5

$153: L=O T(1) *(1-61-$

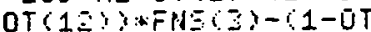

(13) $+F H S(4)$

1EG IF:SYGTHEHF $(4)=$

$F(4)+C X I+K I) * K T * C$

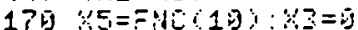

16日 FUEI =1TOZ:S(I)=

-3. $443+$ FNOC1日) 133.5

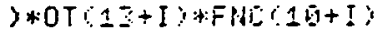

$*: 15+c$

$185: Z=Y 3+\because(I): N E X T$

190 F $(E)=F(E)+Z(1)+$

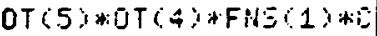

$195: \because 6=3(2)+1) T(7)+1]$

$T(6) * F N 5(2) * C: X 7=3.4$

$13 * F:$ : C $(2)-X 3$

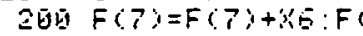

$8)=F(\Omega)+F(F)=F(9:$

$+30(3)$

$24 \bar{G}$ F $(5)=F(E)+F(T)+$

$F(G)+F(\theta)$

250 F 2$)=0 T(2) * F(E)$

2ST IFXTYQTHEHF CO =

$F(3)+x+0 T(9)+3 E+1) T$

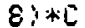

$300 \bar{\sigma})(1)=F(4)-F(3:-$

$F(2)$

$205 \% E=Z E=F P(S)$

$310=(10)==(10:+F H)$

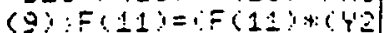

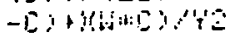

315 FLEI $=2 T 01 T: F C \mathrm{I}$

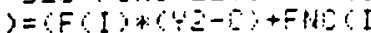

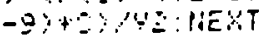

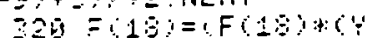

$2-1+3+1+1+2$

$2 \sin =0 \mathrm{~F}=\mathrm{i}=\mathrm{O}$

$79=4-3==40+13$

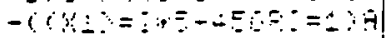

W $-1(X 2)=i * 5+450 \mathrm{E} I=1 ; A$

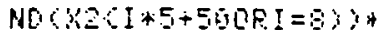

H, 10

$T \because \bar{B}$

STORE 1

FET 1

SLFE $57 \mathrm{BS}$

$419 \quad 34=84+35+1$

420 FORI $=40 T 042: F C I$ T-IHT

$=F(I)+F N S(I-35) * E: M$

EXYT

430 FORI $=44 \mathrm{TO} 45: \mathrm{FCI}$

EXT

448 IFFNISOCOT(10)

THENF $(47)=F(47)+C O T C$

16) -FNEC 3$) \times * C$

450 IFFNC(Z))OT(11)

THENF $(48)=F(48)+(F N)$

(3)-UT(11) $* C$

460 IFF $=$ GTHEN5BO

470 IFXE)

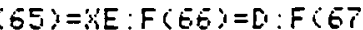

\rangle$=H$

$483 \times X E=0$

5OE IFO $=41$ THENO99

51D IFYI $=0.1$ THE

$: 44=14:$ EDTOSGS

$520 \quad 41=0: 15=15+42$

530 FORI $=25 T O 31: F(I$

$\gamma=F(I)+F(I-21): N E X T$

$540 F(19)=F(19)+F(1$

$: F(21)=F(21)+F(Q): F$

$(Z Z)=F(2 Z)+F(Z)$

550 FUEI $=1$ TUS:FC $2+$ !

$+18)=5(2 * I+17)$ C C $Q 2$

$i+[1): N E S T$

560 FUF $\mathrm{I}=320 \mathrm{TOS}: \mathrm{FCl}$

$y=C F(I):(\mathrm{C} E-4)+F(\mathrm{I}-$

$21)$ *

$570=(46)=6 F(45)+\left(C^{\prime}\right.$

$5-12)+243$ 'TE

561 $3=-1$ IFM $\div 4 T H E$ 1953

E19 FFINT! THECOO "M

OPTH"UAFEIUTDUT"

QZO FOEI=LOTUET:FRI

UT! "F"I F $(I): F C I)=0$

NETT

230 $44=14: 15=0$

$3091=135+22:$ :

$99 \%$ I =15SE 22, ENO

CI $>\mathrm{Fll}-24$

MLWITE: ESTEM

FFOG I $\therefore$.

TIME GEFAILLTS

YEAR Da ?

FEINIT INTERUAL

HES 24 MINE 5 ?

CH STOFE IHTEEUAL

HEE $\perp$ MI HE OS

FUMU ETDFE INTESWAL

HES G MINS E?

FUMU ZEST IRTEFUAL

HF: 9 MIHE

CHAMHE: COEFHULTS

HMALOOOIEITAL

4 EEPSODE TEEHE:

Co: $=600=1$

Pi, 5

$T \div$

ETSE:

E-

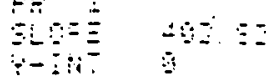

CuC FroUESE 1

A. $C \mathrm{C} \cdot \overline{\mathrm{C}}$

$T A 1$

STOEE 1

FRT 1

SLOFE T72.79

T-INT -456.5E

COO FROEESS 1

Fid 0

$T, A 1$

STOFE 1

FFI 1

$\begin{array}{lr}\text { SLOFE } \\ Y-I M T & -454.98\end{array}$

CGE FROCESS 1

FiO

TAP 1

STORE 1

FFT 1

SLOPE

$\because-I H T$

65. 58

$-458.77$

DQT PEOTESE 1

H. $\mathrm{C}$

$T A 1$

GTOE 1

PET 1

SLOFE ESE?

T-INT -45T. 00

Qud Frones: 1

F.' 0

$T, A 1$

STOEE 1

FFT :

SLOFE

Y-IHT

647.55

COH FROEESE

CDI FFOCESS 1

A.' 1

$T, A$

STOFE 1

FRT 1

SEALE 40

CD. F FELEESE 1 "

H.O

$T, \bar{A}$

STUFE 1

FPT I

SLOFE TS.789

Y-INT 84.59

CDK FFUESE 1

A.' $\mathrm{C}$

$T, F 1$

STHE

FE: 1

柏

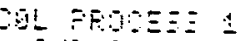

E.

T:

Fition

FF
\rangle$=F(I)+F i N)(I-4 I) * C: N$
$11 / 15 / 82-2 / 28 / 83$

$82 / 83$ HEATING SEASON

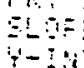

$-5.5$
INIT J

Con Frus

H. 5

$T, 1$

PET 1

SLIFE 220.78

Con FEOEESE 1

A. 1

tition

STOFE 1

FFT 1

SCFE

UFDATE?

STPTUS

$5 B \perp$ FET

502 FET G

SQI Fet 0

$504=5 T$

505 FET 1

50E FFT

507 FET

508 FFT

UEDATE?

DUTFLTT

\# UUTFUT TEFME

UFGATE? 0
$5 T O E E 1$

Y-INT 31 . 
FUHOTION DEFEULTS

\# FUMT:TEK 67

F 4 ETOEE G FRT 1

F ETOEE G PRT 1

$F=$ FRT 1

F 4 STRE G FFT 1

F 5 GTOE 1 FET 1

F E TOE G FFT 1

F 7 STOEE FET 1

$F$ FET 1

$F$ STORE 1 FFT 1

F10 STUEE 0 FET 1

FI1 STURE OU FRT 1

FI2 STURE A FRT 1

FIS STURE G FRT 1

FI4 ETUEE

F15 ETURE

F16 STURE O

Fis STURE G

F15 STORE

F19 TOFE 0

F2G STLRE

F21 STOFE 0

FE GTOPE 0

FO STOSE $\theta$

F24 STOFE

FOS ETOE

FEE STOFE G

FE GTOPE

F28 STUEE 0

FEO ETUEE

FE STRE

FISTOEE

FE STOES

F Z ETORE

FY GTGRE

FES STOEE

FOS TOEE

F37 STOFE

F3E STORE 0

FSO STQRE

F40 STOAE O.

F+1 STORE

F42 STUFE

F43 STUFE $B$

F44 STLFE B

F45 STURE $\theta$

FAE STUE

F4T STOFE

F4S STURE

F49 TURE

F5U DTURE D

F51 STOFE 0 .

F.2 3 TORE

FSI STOFE 0

FS4 STORE

F5S STURE O

F5S STORE $B$

FST ETORE $B$

FSE STINEE O

F59 GTURE

FGU STURE $B$

FS1 ETCRE U

FSQ STERE O

F5S STORE O

Fo4 ETLEE

FE5 STIRE

FSO STCED

FET

FRT 1

FRT 1

FET 1

FET 1

FFT 1 .

FRT 1

FPT 1

FRT 1

FRT 1

FPT 1

FFT 1

FET 1

FrT 1

$F=T 1$

FET 1

FET 1

FET 1

FRT 1

FRT 1

FET 1

FतT 1

FET 1

FRT 1

FRT 1

FRT 1

FFT 1

FFT 1

FRT 1

FRT 1

FRT 1

FRT 1

FRT 1

FPT 1

FRT 1

FFT 1

FRT 1

FRT 1

FRT 1

PRT 1

FRT 1 .

FRT 1

FFT 1

FRT 1

FRT 1

FRT 1

FRT 1

FRT 1

FRT 1

FPT 1

FRT 1

FRT 1

जT 1

FG FTEE B. =RT 
LIST : : :

1 :EPC:I=LSE(20): \$90 HE:TT

- 10 OIMOTCIS)

$1=1 \equiv-29: 41=01$

i. 15 FORI $=255 T$ TSTEF

4:FOKET $9051, I$ : NEXT

20 OT (1) $=25.2$ : OTC. $\Rightarrow=.55: 0 T(3)=.75$

30 OT $(4)=0:$ OT $(5)=0$ OT $(5)=0 \cdot \because \therefore \cdot \therefore \cdot \therefore$

40 OT $(?)=0: 0 T(8)=0$

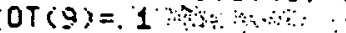

-50 OT $(10)=58: 0 T(11$

$=75: 0 T(12)=391$

60 OT $(13)=1: 0 T(14)$ $2: 0 T(15:=2$

70 OT $(15)=2: 0 T(1)=$ 6 . $\because 140$

SO OT $(18)=0$ :OT (19)

0 O $\therefore$ in

$99: I=U E R(24)$ I
110 IFY3C -1 THEH1S

110 IFY3C -1 THEPIS

=0:NEXT

$140 \times 4=0: 42=0: 43=0$

15D $\because \because E=C F H C(4)+F H O$

5) +FNE(6)?

$151 \times 1=F N C(4): \therefore 2=F$

C(?)

$155 \quad 42=12+0$

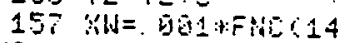

CL:XT $=X B-F H C Q 3 ;: I F:$

CQT $\therefore$ ENMT $=\overline{0}$

$158 \quad \because I=60 T(18)^{-2} 2 x$

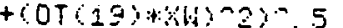

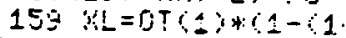

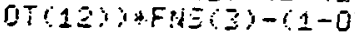

(I) I FFHC 4 )

IES IFXTSOTHENFA

$F(4)+(Y L+X I) * Y T *:$

$170 \because 5=F H Q 10):=$

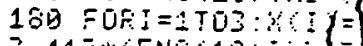

$-3.413+C F M(1 B+3)=$

ᄀ*OT $113+I$ I $+F N D C 16+I)$

$*: 5 * 0$

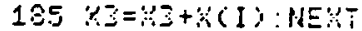

$130 \quad F(E)=F(\varepsilon)+R(1)+$

OT $65 * 0 T(4) * F N=C 1$ )

$\because 195 \therefore 6=\because(2)+10 T(7) * 0$

T(6)+FMECZ)*C:X7=广. 4 13 HWF (G)-X

200 F $(7)=F(7)+Y S: F$ $\varepsilon)=F(\theta)+M F: F(g)=F(g)$ $+3(3)$

$24 \overline{1})(5)=F(\theta)+F(T)+$ $F(\theta)+\theta \theta)$

$250 \mathrm{~F}(2)=0 \mathrm{~T}(2) \mathrm{H} F(\theta)$ 2 IF IFTOETHEHFQS

$F(Z)+M T+Q T Q S) K E+B T$

8) +10

$305 F(1)=F(4)-F(3)-$

Fi?

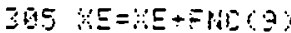

. $210=20)=F(10)+F M$

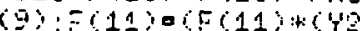

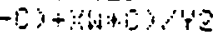

¿15 FOF! = 2 TO1F:

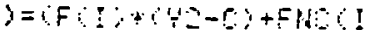

-

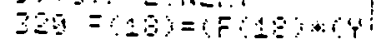

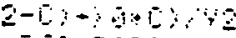

$80=0:=-10$

$87=0 \div=2-8+1)$

$-692=0=-50=1=1$ A

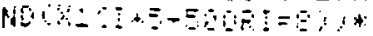

$\mathrm{C}$

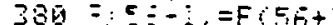
$-(C X 2)=I+5+450 F I=1$ HOCXOI $5+590 F I=5)$

CoTE FF BE:

A 0 O

$T, \overline{1}$

STOSE 1

FPT 1

SLORE

$Y-I N T$

$410 \quad Y 4=84+Y 5: 10$

420 FOFI $=40 T 104 \Omega: F$.

$\lambda=F(I)+F N S(I-35)+L$

EXT

430 FORI $=44045: F 1:$

$\lambda=F(I)+F N C(I-4 Z)+10 \because$

EXT

446 ifFNo 3 )

THEHF $(47)=F(47)+\left(0^{\circ}\right.$

1B) $-F H C(\Omega) ; * C$

450 IFFROS3)

THENF $(43)=F(48)+(F I$

(5)-QTC $11:) * C$

460 IFF = OHENSBD

470 IFUEYFCESTHEI

$(65)=$ YE: $F(E S)=0: F C$

$y=\mathrm{H}$

$489: X E=0$

505 IF $=$ THENGQO

510 IF

$: 44=n:$ GOTO999

$520 \quad Y_{1}=0: 45=45+\%$

530 FORI $=25 T 0=1: F$.

\rangle$=F(I)+F(I-21): M E X$

$540 F(29)=(19 i+F$,

) $F(21)=F(21)+F(2)$

(23) $=723)+7(3)$

550 FOPI $=1 T 03: F(2)$

$+18=F(2+I+2) C(F)$

iC1): $\mathrm{HE} E \mathrm{X} \mathrm{i}$

560 FOF $\mathrm{I}=\mathrm{OTOSO}: \mathrm{FI}$

$x=C F(I)+C=-2)+F$

$21)+2$ HS HEYT

5P日 F $\langle 4 E:=\{F(4 E) \cdots$

$5-12)+4+4,5$

$8615 \%=-1:$ IF $Y=44 \mathrm{Tl}$ NOS

Q10 FRIMT!TAEC20!'

DHTH"IS"FFEMTOUT"

OZG FUFI=13TOGT:F

:UT:"F"I;FCI :FUI

VIESTT

$030 \quad 4=14: 4=0$

SOS I=USFOZ2: ENO

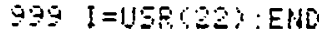

$6>\sin -24$

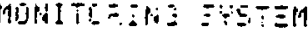

FFOI ?

TIME DEFAULTS

UFCITE: 1

HEAR S2 ? 3

FEINT IUTEEUAL

HFE 24 MIMS I ?

CH ETURE IMTEFUtiL

HRS I MINE मे?

FURL STORE IUTESUAL

HES ODIHE 可?

HES 6 HIHS 0 ?

CHFHHEL DEFELLTS

AMALOGOTITITAL

EXARI!:E?

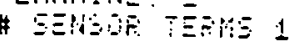

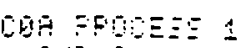

सC:

$T ; \mathrm{A}$

GTHE

Fet 1

经过

COE FFOUESS 1

FiC $\mathrm{O}$

$T \because A$

STOFE 1

FPT 1

COC FRUEESE 1

A. $O$ O

$T, F 1$

STUFE 1

FFT 1

CQE PROLESE 1

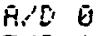

$T, A 1$

STORE 1

FRT 1

Cof FoOUES: 1

But

$T \because 1$

GTOEEI

FFT 1

Cog PEOEESE 1

H.' 6

T:P 1

STOEE 1

FET i

GLFE

$\checkmark-I N T$

CQU FROLESE

CUI FROCESS 1

A I 1

$T \therefore \overline{1}$

FRT 1

OFFEET

COJ FROEESE 1

A. $\mathrm{OB}$

$T, 1$

STOAE 1

FET 1

CDE FEOEES 1

A.S 6

Tit

STOE:

FFT' 1

$\because-I H T$ T

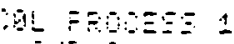

Fis 6

? 1

$-15 \equiv$

INIT

$3 / 3 / 83-5 / 22 / 83$

Fing $82 / 83$ HEATINGASON

SLOEE T72.79

Y-INT -45E.56

SLOFE E54.98

$Y$ IIIT -455. 75

SLOFE E.S2.019

$\because-I N T \quad-458.77$

SLOEE ESE.?

Y-INT -457. 88

547.65

$5 T 0, E$ i

SLOEE PZ.789

४-InT 8.529

SUFE 200

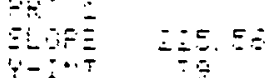

CQM FFOEES 1

A.D 5

T, 1

STOEE 1

FRT 1

SLUPE 220.78

$Y$-INT, 31

CéPR FROCESS 1

A. 1

T:

STOEE 1

FFT 1

SULE $\cdot 238$

OFFSET

UFDATE?

STATUS

EKAIINE? 1

SDI FFT

502 FRT

5 STET

SUA FET 1

SES FET 1

50 FET

507 FT 6

5QS FAT

UPLATE:

OUTFUT

EXATEHE? 1

\# OUTFUT. TEEMS 
FUHITION DEFAULTE

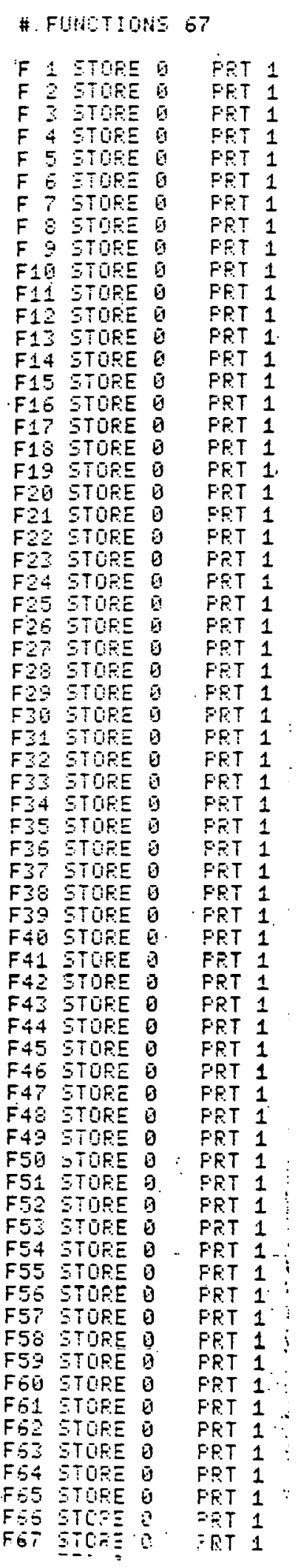


SITE SK

Mr. \& Mrs. Jerry Sams

1055 th Street

Black Mountain, NC 28711

INIT2 Software Installed: 02/03/83 


\section{SITE SUMMAZY SHEET}

Site Identffication Number SK1
Inst. Contr. Naze ESG, Inc.

Address 2231 Perimeter Park

\section{Location:}

Town Black Mountain, NC

Latieude 3503'

Longi=ude $82^{\circ} 3^{\prime}$

Elevaion $217^{\prime}$

Nearest Neather Station:

Asheville. NC

\footnotetext{
Build1nğ:

Type of Building

General Type of Construction

Ground Coupling

Passive/Hybrid systea(s):

Dominant Heating System Type

Dominant Heazing System Area

Other Systea Type

Other Systen Area

Nighe Insulation

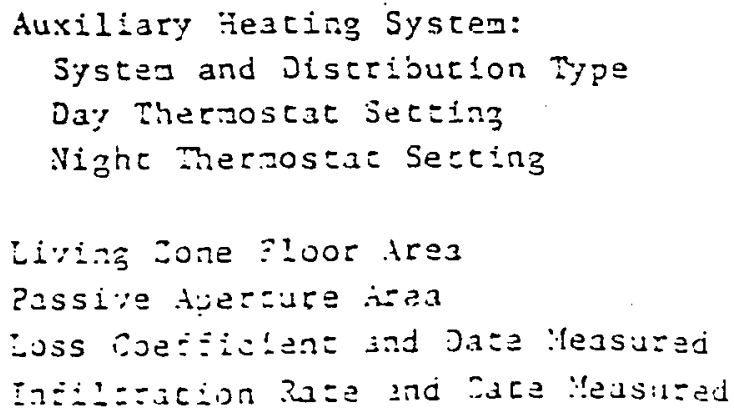

One story residence Wood frame and veneer STab on grade

Direct gain/Trombette walls $87.9 \mathrm{ft}^{2}$

$\mathrm{N} / \mathrm{A}$

N/A

Yes. window quilts (south only)

Baseboard resistance heaters $68^{\circ}$

$68^{\circ}$

$893 \mathrm{ft}^{2}$ $87.9 \mathrm{ft}^{2}$ L=207.8 Btu/hrof: $2 / 2$ and $2 / 3 / 83$ $.115 \mathrm{in} 3 / \mathrm{sec} \cdot \mathrm{a} 4 \mathrm{~Pa} 8 / 1 \mathrm{n} / 82$ 


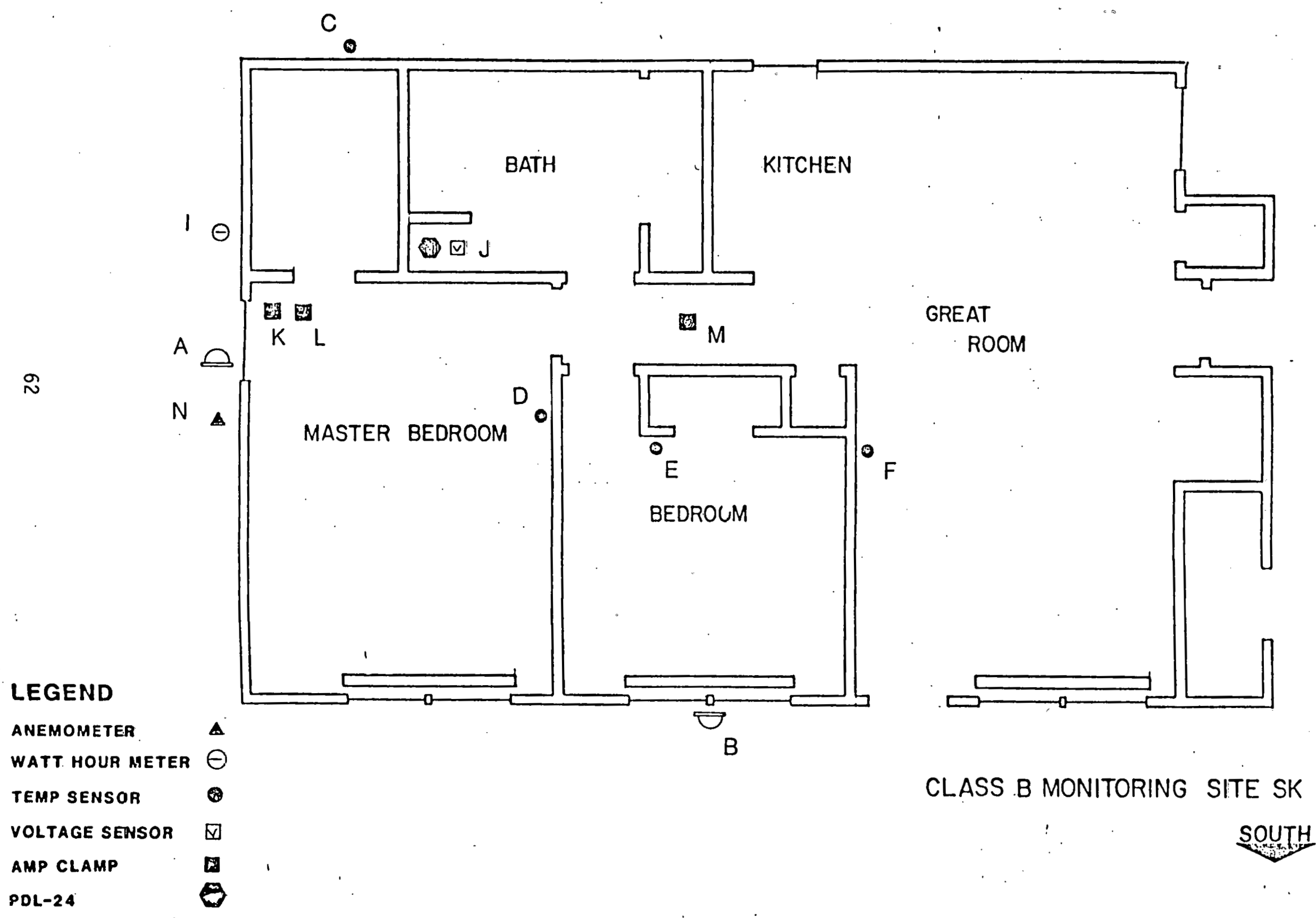




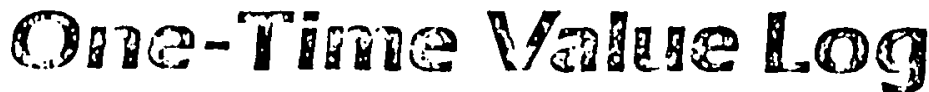

Sile Black Fountain, JC Uperalor D.H. Gustashaw Site Code SKI Update Program Name INITK Date INII2

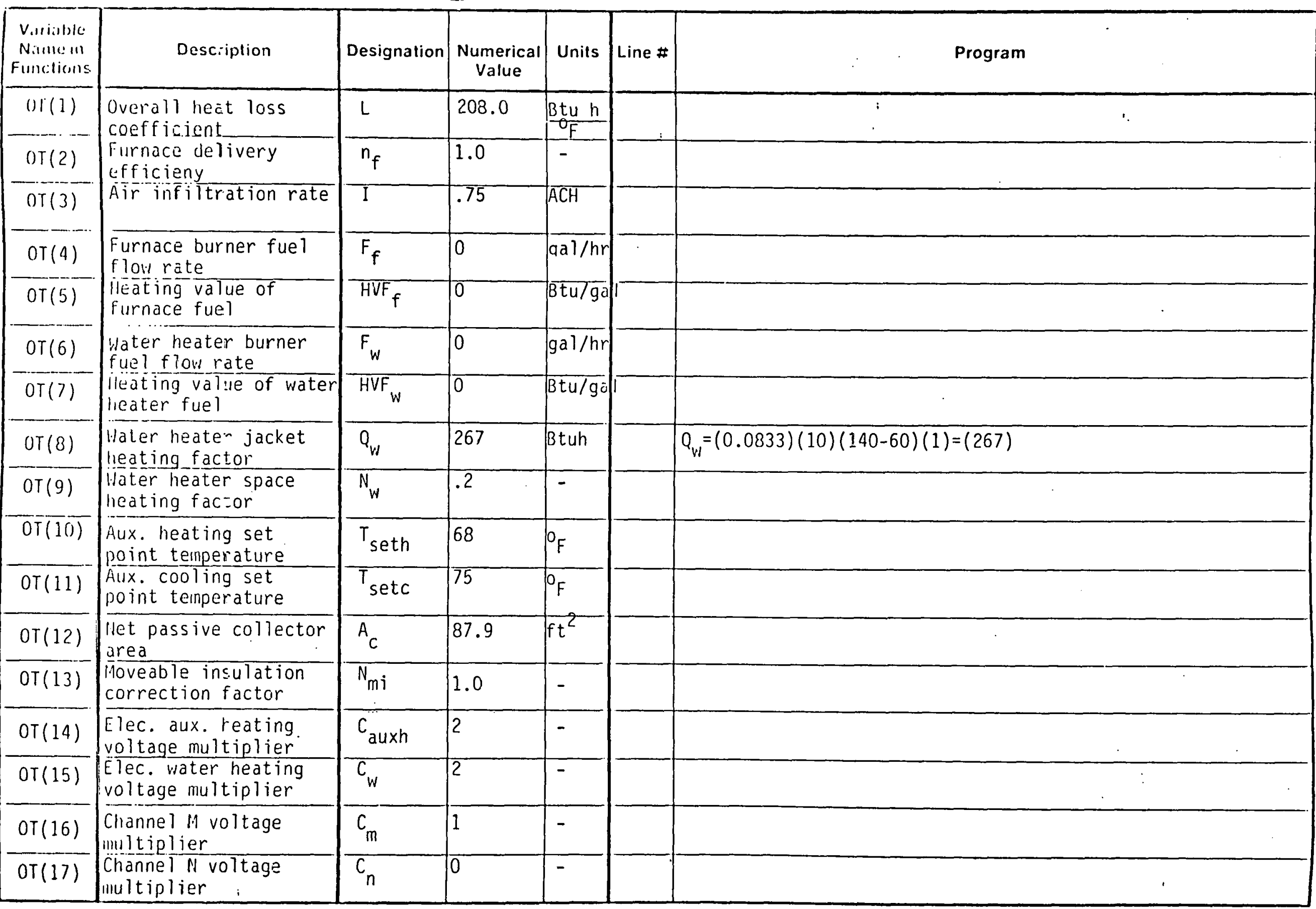




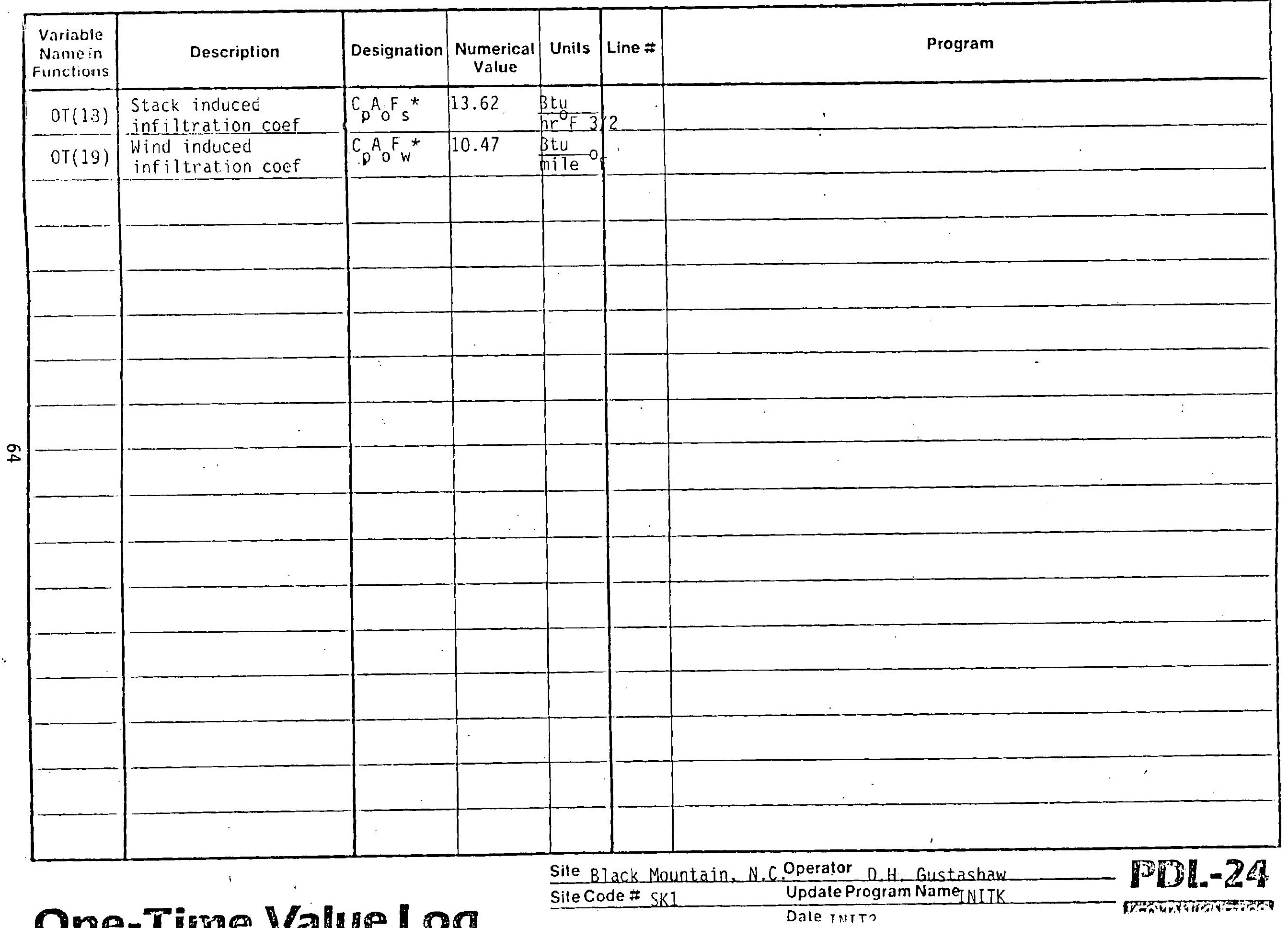




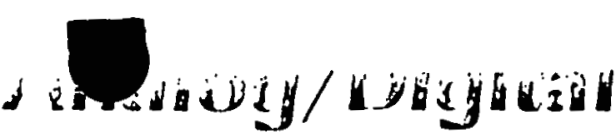

Champosellog

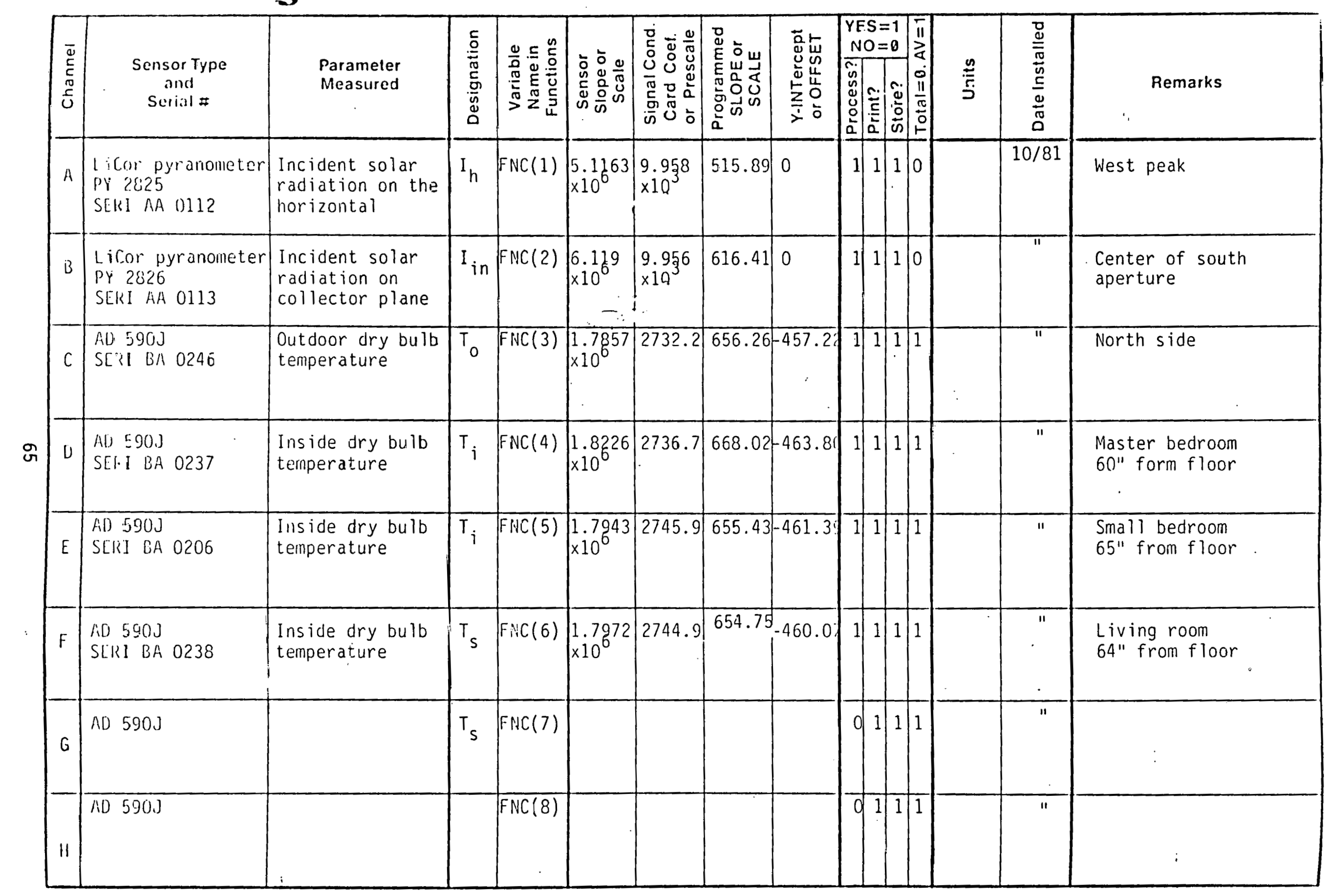




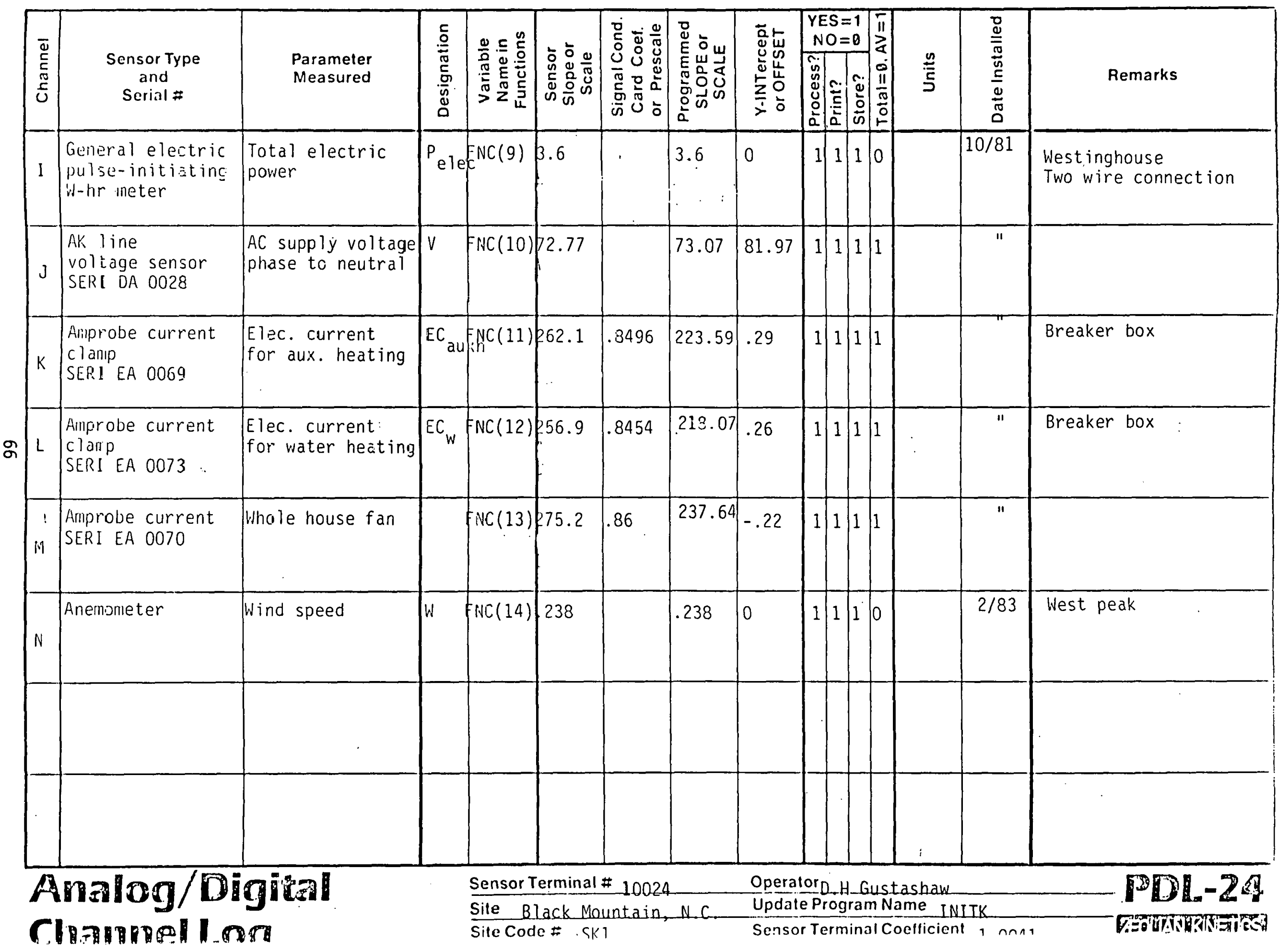


Sensor Terminal * 10024

Site Black Mountain $\mathrm{NC}$

Operator D. $\dot{H}$. Gustashaw

Update Program Name INITK

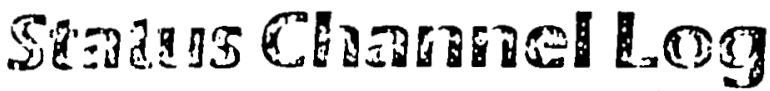

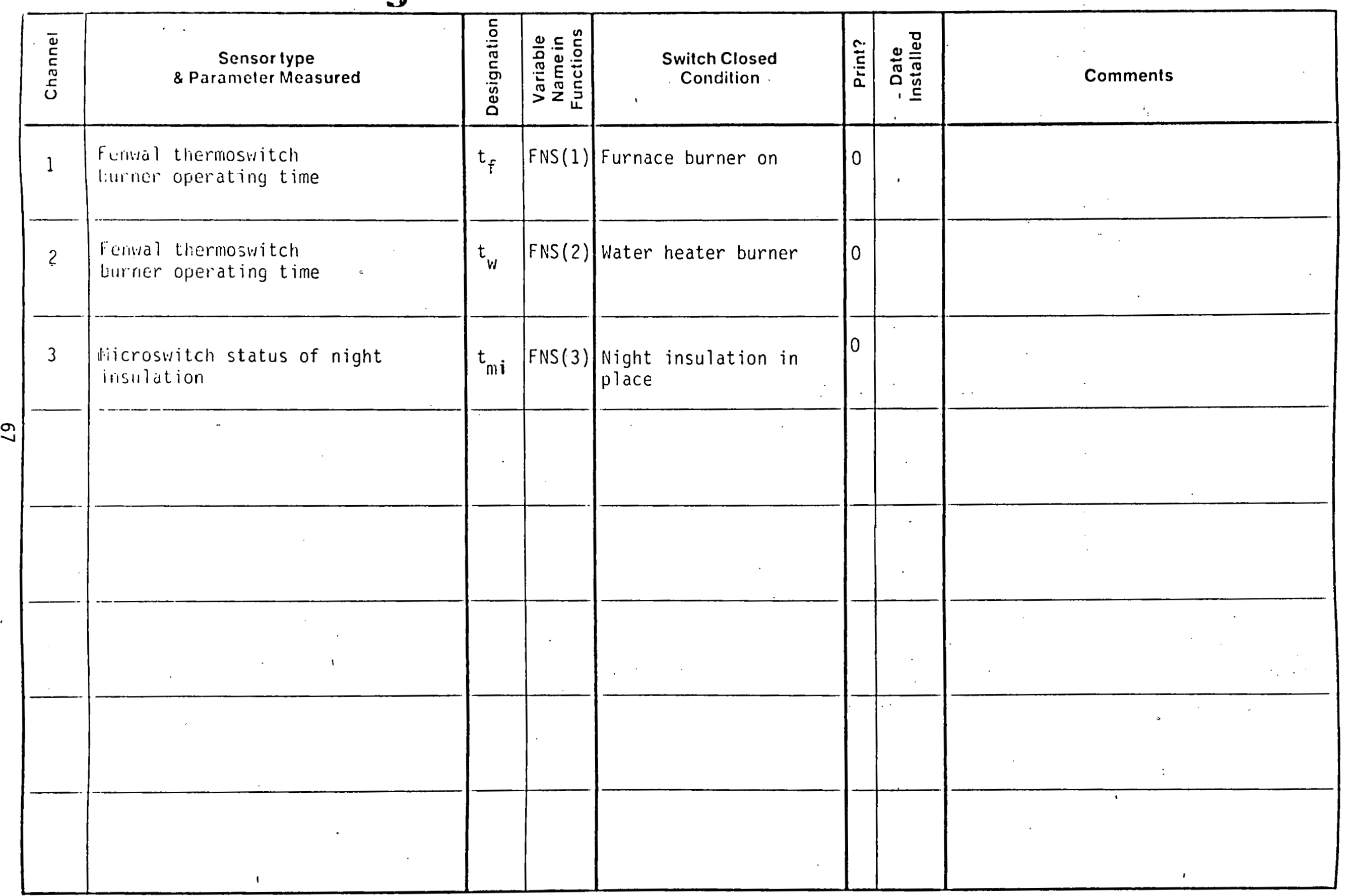


LIST

1 : END I= IEe 20

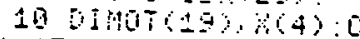

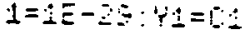

15 FOC I OESTOSTEF-

4:FURE4GGE: I:HEY

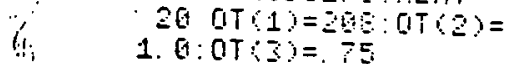

30 OT $(4)=8: 010(5)=0$.

$0 T(6)=0$

46 OT $(7)=6:$ aT $(3)=26$

$7: 07: 5)=2$

50 (IT $(19)=68: 0 T(11)$

$=75: 0 T(12)=37.9$

ह日 aT 12$)=1.0: 0 T(14$

$\lambda=2: 0 T(15)=2$

6

$7(4$ OT (1) $=1: 0 T(17)=$

80 0T(18)=12.82:0T(

19) $=10.47$

$99: I=115 R(24):$

11日 IFHTC-ITHENA 50

12G FOFI $=1 T 018: F(i)$

$=0: N E X T$

$140 \quad \times 4=0: Y 2=0: Y Z=0$

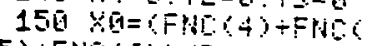

5) $+F N(8)=$

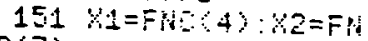

C. (7)

$15512=2+0$

$157 \quad X 4=601+F)(14)$

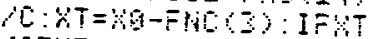

GTHEMUT =

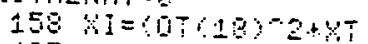

$+(01419 y+4)$ o $=$

153 KL=0T:13:1-1-

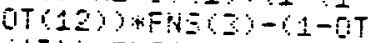

$43)$ and 64

AEG IFRTOETHEHF $4=$

$F(4)+C Q+Y T)$ H T

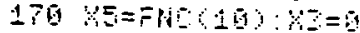

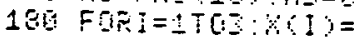

-3. 42+CFNCAG+1)=

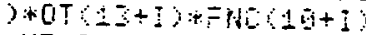

$* \times 5 * \mathrm{C}$

$185 \quad \%=28+\because 1) \cdot M=\%$

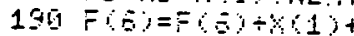

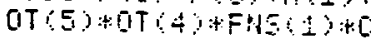

$195 \quad \because 6=40+\mathrm{CT}=2+0$

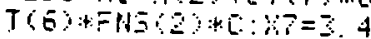

12*FHi 9$)-4$

$200 F(T)=F=)+B E:=0$

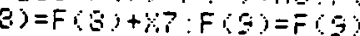

$+4(2)$

$240 F(5)=5(6)+5(7)+$

F(B)+F

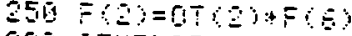

200 !

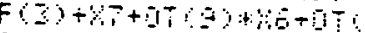

(a) : $: C$

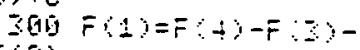

$F(2)$

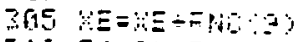

$20 \bar{r} 10==20+=$

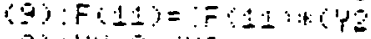

$=0)+(1)+10)=$

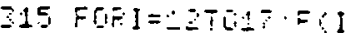

$y=(F)$ I Y t

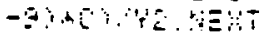

$00=0=0: \because 200$

$2-c 1+2-2$

$06=8=-1$

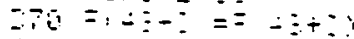

$-16-2=0=0=0$

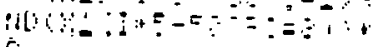

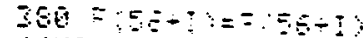

$-66 x=2$ it $5+4000=10$

No $\left.\left(x_{2}\right)+5+500 \mathrm{~F}=3 \mathrm{y}\right)$

$\mathrm{C}$

396 NEYT

$416 \quad X_{4}=X_{4}+X_{1}+C$

42 FCIRi=4OTGCZ:F I

$\partial=F(I)+F H S(T-5)+C \cdot P$

EQT

$456=O F I=4+T 045: F I$

)$=F(I)+F N C(I-4) ;+C: N$

EXT

440 IFFNOCOCOT(16)

THENF $(4 T)=F(4)+O 0 T\}$

16) $-F(10(2))+C$

450 IFFHCOZ)OT(11)

THEN $=48)=F(48)+C$ FNO

(3) -0T (11)) $\mathrm{k}$ :

466 IF $=0-4 E N 50$

I 470 IFUEYESESTHERF

$(65)=Y E: F(E S)=0$ D $F(E)$

) $=\mathrm{H}$

$480 \quad Y E=0$

500 IF $0=1 T H E N 959$

510 IFYI ATHENTI=0

Y4=17: GOTC 955

526 1 $1=0$ : $5=15+2$

520 FOFi=25T0S4:F

$\gamma=F(j)+F(I-21): N E S T$

$546 \quad: 19)==19)+F(1$

)$: F(21)=F(21)+F(2): F$

$(2 Z)=(23)+(2)$

550 FIFI=1T0 F

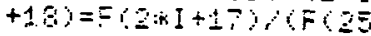

$X+C 1): H E Y T$

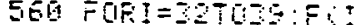

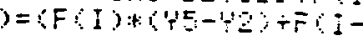

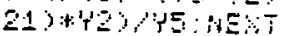

576 F 4 G)=(Fits)

$5-12 y+4+4=$

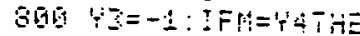

1998

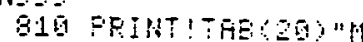

CONTH"TA "PRISTOG

820 FCIRI IOTLET:FEI

NT!"F" $F: F(I): F(I)=Q$

NIEXT

650 :4=1:15: $=0$

999 I = 1SR $3=5$

$9491=(1 S R 202):$ ENO

SCPDL -24

MUNITLEING SRSTEM

COPUFIEHT 1986

GE CIL IAP KIHET:S

BX 196 FFOU RI 18501

FFIIG !

TIHE DEFGUISE

TEAF $\$ 2 ?$ ?

FFINT INTEEUAL

HPE 24 MINE $\mathrm{A}$ ?

CH ETURE YUTEFUAL

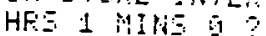

FURL ETHE IRTERTL

HFE E UIMS

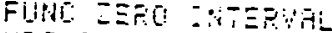

HFE 5 调H 6

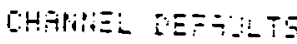

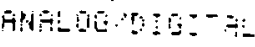

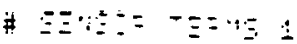

U⿻

$2 / 3 / 83-5 / 19 / 83$

Al:

$T$ P

STORE:

FFiT I

SLCIFE 5 -INT

82/83 HEATING SEASON

1CEE FEOCES:

Aits

The

STGF: 1

PFIT 1

SLOFE EIE. 41

COC PROCESS

A $O D O$

STORE I CODL PROCEES:

FPT

SLORE

Fi, 1

$T, A 1$

STOFE I

PRT 1

SLOFE 2006

COA FrOCES:

$\mathrm{A} / \mathrm{O}$.

TYi 1

ETOFE:

FET 1

SLOFE

1

P-Tit -

COE FEOESE 1

A.

TYA

STUEE 1

PFi:

SLQFE

$655 \div 2$

CQR FFOEESI

คด

TIF 1

STORE 1

PFT 1

SLOFE EESTE

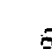

COC FROEESE

CEH PFEILESE 0

COI FFGEESE:

H. 1

T/A

STOEE 1

PFiT 1

SCHLE 3.5

QFFEET

CQI FFUCES:

A. 6

if 1

STEE 1

FFT

SLliFe 75 BE

T-INT 89

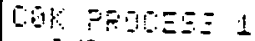

Fit

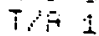

-

FF:

0
$y-10$
Con FFUEESE

की

TH 1

STOFE 1

PRT 1

SLOFE 23.84

Y-IVT -2

Con FPOCE三E 1

fir 1

TY E

STURE:

FF 1

GFFE

UIFDATE? O

STATUS

E11 PFi

502 FFT

SEIPFT

SBIA FET

505 FET

SUE FRT 9

SET PFT E

$3 G 0$ CFT

UPQBTE? -1 
FUMETION OEFAULTE

\# Flifotrotis B?

F I ETOEE G ERT 1

F 2 STOEE G FRT 1

$F=$ ETOEE OPT 1

$F$ STUFE O FET 1

F 5 TORE Q F FTT 1

$F E$ TIRE O FFT 1

$F 7$ ETOEE 1 FET 1

$F$ ETURE G FRT 1

$F G$ STRE - FET 1

F10 STORE G FET 1

FI1 STUEE O FET 1

F12 STURE G FRT 1

F1S STURE E FRT 1

FIA ETOEE E FET 1

F15 TURE O FRT 1

F1E ETORE E FRT 1

Fi? STURE 0 FFT 1

F1S STORE 0 FFT 1

F19 GTORE O FRT 1

F20 STUFE O FRT 1

F21 STORE G FFT 1

F2 GTURE 0 FFT 1

FES STURE $Q$ FRT 1

F24 GTORE G FPT 1

F25 STURE 0 FET 1

F2E TUEE A FET 1

FT ETRE G FRT 1

F2E ETREE 5 FET 1

FQS ETOEE O FET 1

F30 STCRE 5 FRT 1

FS1 STORE O FRT 1

F2 ETORE E FFT 1

F3S STORE O FRT 1

FU4 ETORE G FET 1

FE ETORE G FRT 1

F3E STORE E FRT 1

F3T GTORE ब FRT 1

F3S STORE Q FET 1

F39 STOEE G FRT 1

FAO STURE O. FFT 1

F41 STOEE B PRT 1

F42 STDRE O FRT 1

F4 STOFE O FRT 1

F44 STUFE 0 FRT 1

F45 GTURE O FRT 1

FAE STUELE E FRT 1

F47 STURE 0 FRT 1

F48 STURE 0 FRT 1

F43 STORE O FRT 1

FSU DTUEE O : FRT 1

F51 STORE $O$ FRT 1

F52 ETUFE Q FRT 1

FSE ETUFE 6 FRT 1

F54 STLFE E … FRT 1.

F.5. STORE B FFT 1

F.5 STOFE $B$ FRT 1

F5? ETURE $\theta$ FRT 1

F5O ETORE $O$ FRT 1

F5' ETLIRE B FRT 1

FGO ETERE B FRT 1

FDI ETGRE O FRT 1

F52 ETLFE 0 FRT 1

FSE ETLRE D FRT 1

F54 ETLE O FET 1

FES ETUFE U FRT 1

$F=5 T L O E$ ? $=Z T 1$

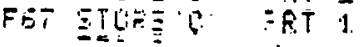




\author{
SITE SL \\ Mr. \& Mrs. Robert Greatorex \\ 4950 Essex Circle \\ Stephens City, VA 22655 \\ INIT2 Software Installed: $11 / 20 / 82$
}




\section{SITZ SUMMABY SHEET}

Site Identfication Number SL1
Inst. Contr. Naze ESG, Inc.

Address
2231 Perimeter Park

Suite 11 Atlanta, GA 30341

Location:

Town Stephens City. VA

Latilude $39^{\circ} 1$

Longi=ude $78^{\circ} 1^{\prime}$

Elevazion $750^{\prime}$

Vearest weather Station:

Winchester, VA

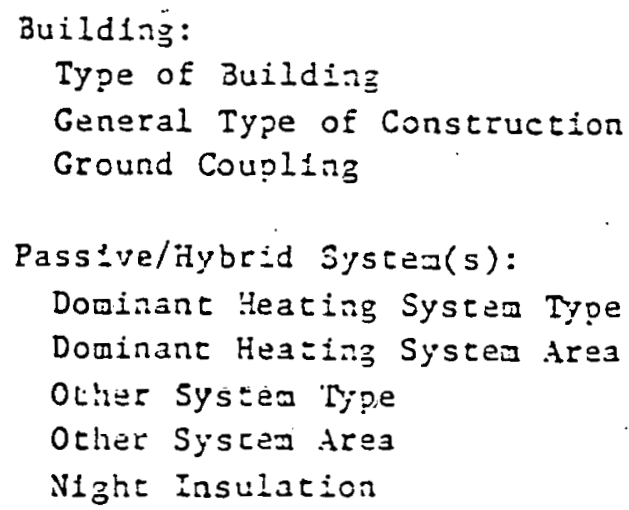

Auxiliary theariag Syscem:

system and Distrijution Type

Da: Theraostat jet:ing

Nigit Therzosta: Setting

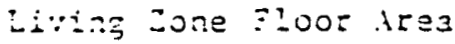

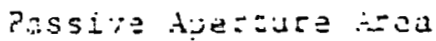

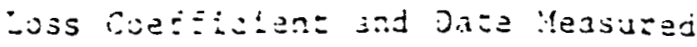

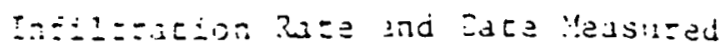

One story residence

Wood frame

Slab en grade

Water wall

$210.5 \mathrm{ft}^{2}$

$N / A$

$N / A$

Yes, window quilts on all windows

Resistance baseboard heaters $65^{\circ} \mathrm{F}$

$650 \mathrm{~F}$

$1200 \mathrm{ft}:$

$210.5 \mathrm{ft}^{2}$

$L=177.6$ Btu/hrof: $1 / 29$ and $1 / 30 / 83$

$.045 \mathrm{H}^{3} / \mathrm{sec} \mathrm{A}_{4} \mathrm{D}_{2} \quad 8 / 12 / 32$ 


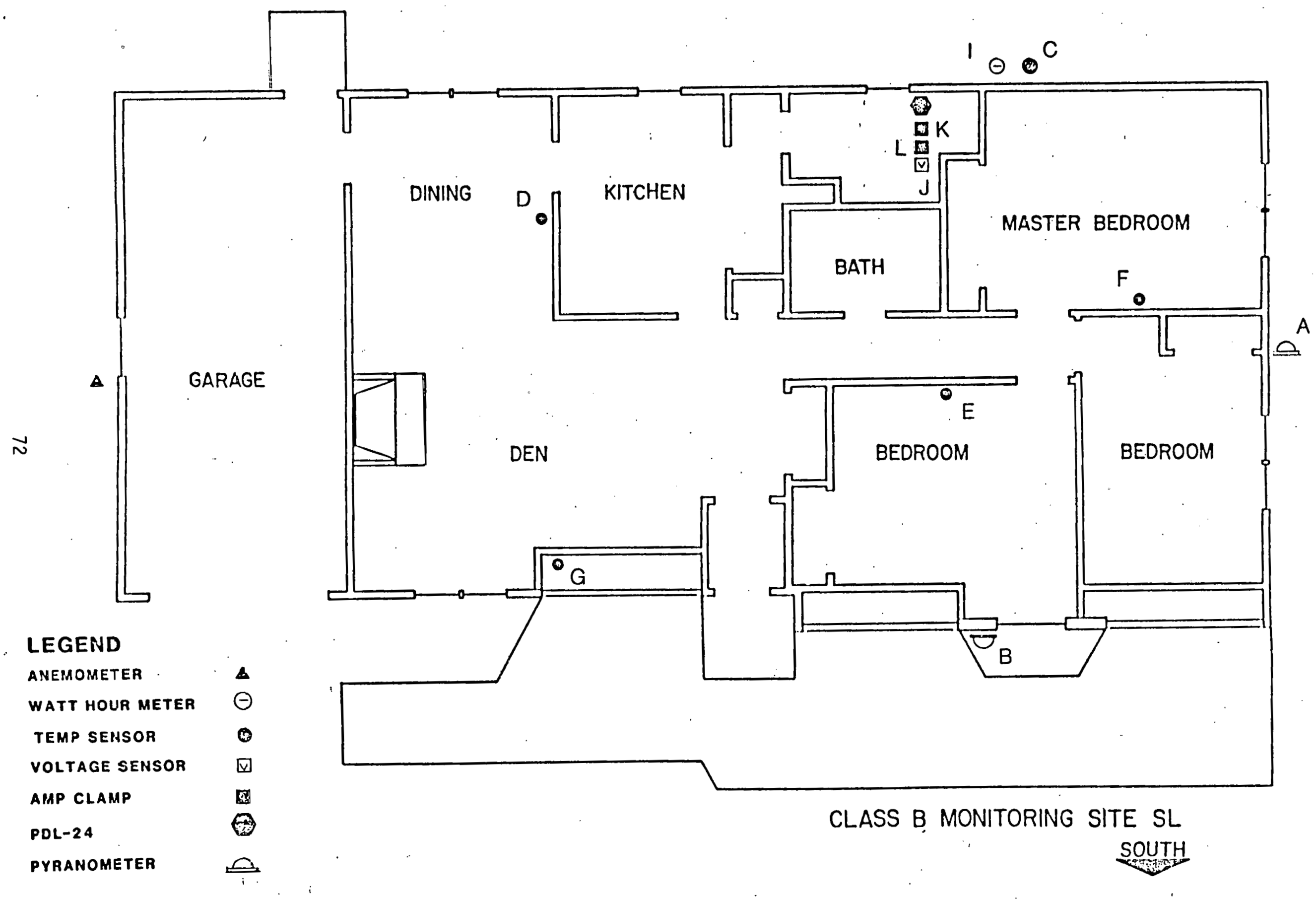


Sile Stephens City. VA Site Code

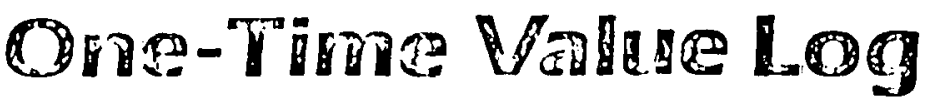

\begin{tabular}{|c|c|c|c|c|c|c|}
\hline $\begin{array}{l}\text { Variable } \\
\text { Name in } \\
\text { Functiors }\end{array}$ & Description & Designation & $\begin{array}{c}\text { Numerical } \\
\text { Value }\end{array}$ & Unils & Line & Program \\
\hline UT(1) & $\begin{array}{l}\text { urerall hedt loss } \\
\text { coefficient }\end{array}$ & L & $\begin{array}{l}449 \\
178 \\
\end{array}$ & $\frac{B t u h}{O E}$ & & $\begin{array}{l}11 / 20 / 82-1 / 29 / 83 \\
1 / 29 / 83-4 / 30 / 83\end{array}$ \\
\hline OT (2) & $\begin{array}{l}\text { Furnace delivery } \\
\text { eficiciency }\end{array}$ & $n_{f}$ & 1.0 & - & & \\
\hline OT $(3)$ & ir inf iltration rate & I & .75 & $\mathrm{ACH}$ & & \\
\hline OT (4) & $\begin{array}{l}\text { Furnace burner fuel } \\
\text { flow rate }\end{array}$ & $F_{f}$ & 0 & gal/hr & & \\
\hline OT $(5)$ & $\begin{array}{l}\text { lleating value of } \\
\text { furnace fuel }\end{array}$ & ${ }^{H V F} F_{f}$ & 0 & $\overline{B t u / g a}$ & & \\
\hline $\operatorname{OT}(6)$ & $\begin{array}{l}\text { Water hater burner } \\
\text { fuel f low rate }\end{array}$ & $F_{W}$ & 0 & $\overline{g a l / h r}$ & & \\
\hline $\operatorname{or}(7)$ & $\begin{array}{l}\text { Whating vaule of water } \\
\text { heater fuel }\end{array}$ & $H V F=$ & 0 & $\overline{\beta t u / g a}$ & & \\
\hline $\operatorname{OT}(8)$ & $\begin{array}{l}\text { Water lieater jacket } \\
\text { heater factor }\end{array}$ & $Q_{w}$ & 303 & Btuh & & $Q_{w}=(.0833)(52 \mathrm{gal})(.70)(1)$ \\
\hline OT $(9)$ & $\begin{array}{l}\text { Water heater space } \\
\text { heater factor }\end{array}$ & $N_{W}$ & .2 & - & & \\
\hline OT (10) & $\begin{array}{l}\text { iux. heating set } \\
\text { boint temperature }\end{array}$ & $T_{\text {seth }}$ & 65 & ${ }^{0} \mathrm{~F}$ & & \\
\hline OT $(11)$ & $\begin{array}{l}\text { iux. cooling set } \\
\text { point temperature }\end{array}$ & $T_{\text {setc }}$ & 75 & ${ }^{0} \mathrm{~F}$ & & \\
\hline $0 T(12)$ & $\begin{array}{l}\text { liet passive collector } \\
\text { frea }\end{array}$ & $A_{C}$ & 210.5 & $f t^{2}$ & & \\
\hline OT (13) & $\begin{array}{l}\text { loveable insulation } \\
\text { inrrection factor }\end{array}$ & $N_{m i}$ & .97 & - & & . \\
\hline OT $(14)$ & $\begin{array}{l}\text { lec aux. heating } \\
\text { oltage multiplier }\end{array}$ & $c_{\text {auxh }}$ & 2 & - & & \\
\hline OT(15) & $\begin{array}{l}\text { lec. water heating } \\
\text { oltage nult iplier }\end{array}$ & $c_{w}$ & 2 & - & & \\
\hline OT $(16)$ & $\begin{array}{l}\text { hannel ll voltage } \\
\text { mltiplier }\end{array}$ & $C_{m}$ & $p$ & - & & \\
\hline$\overline{O T}(17)$ & $\begin{array}{l}\text { hannel N voltage } \\
\text { iultiplier }\end{array}$ & $c_{n}$ & 5 & - & & 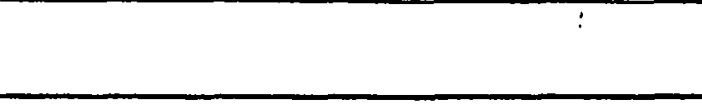 \\
\hline
\end{tabular}

Operalor U.H. Gustashay Update Program Name INITL

$$
\text { cintiplier a. }
$$




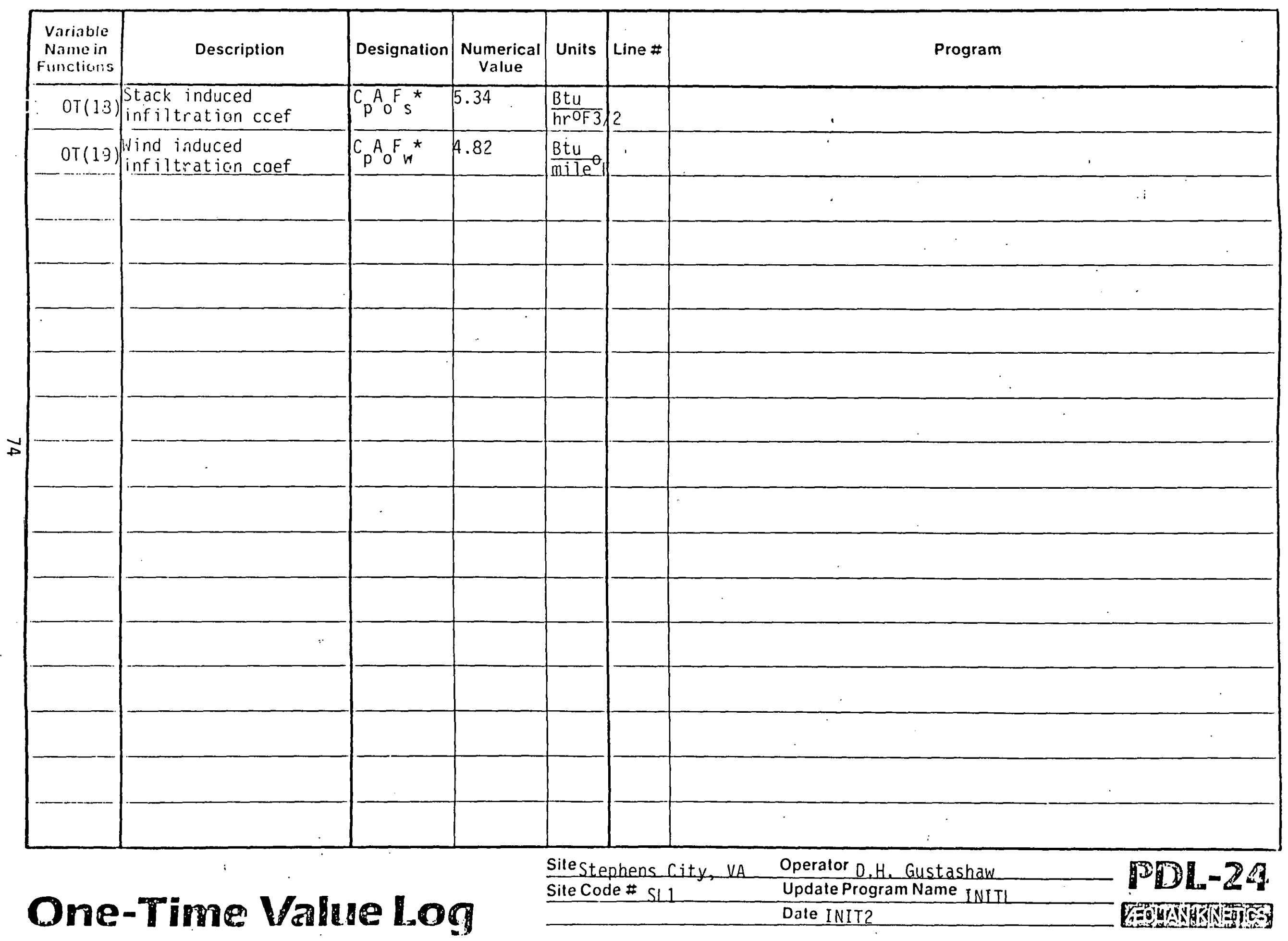




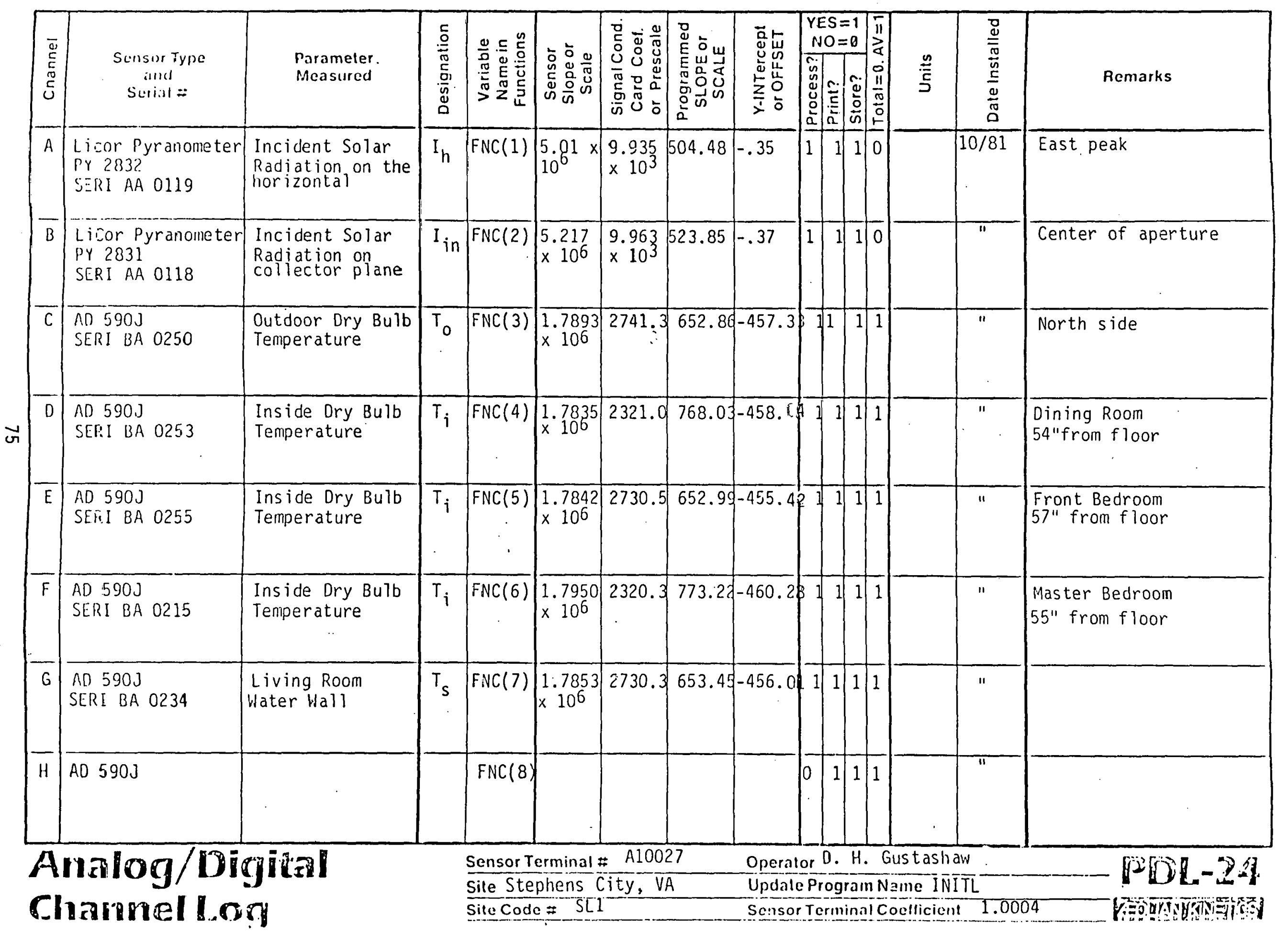




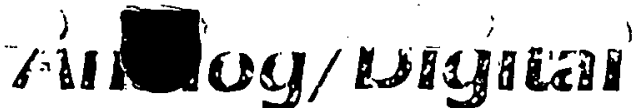 SInamuel Log}

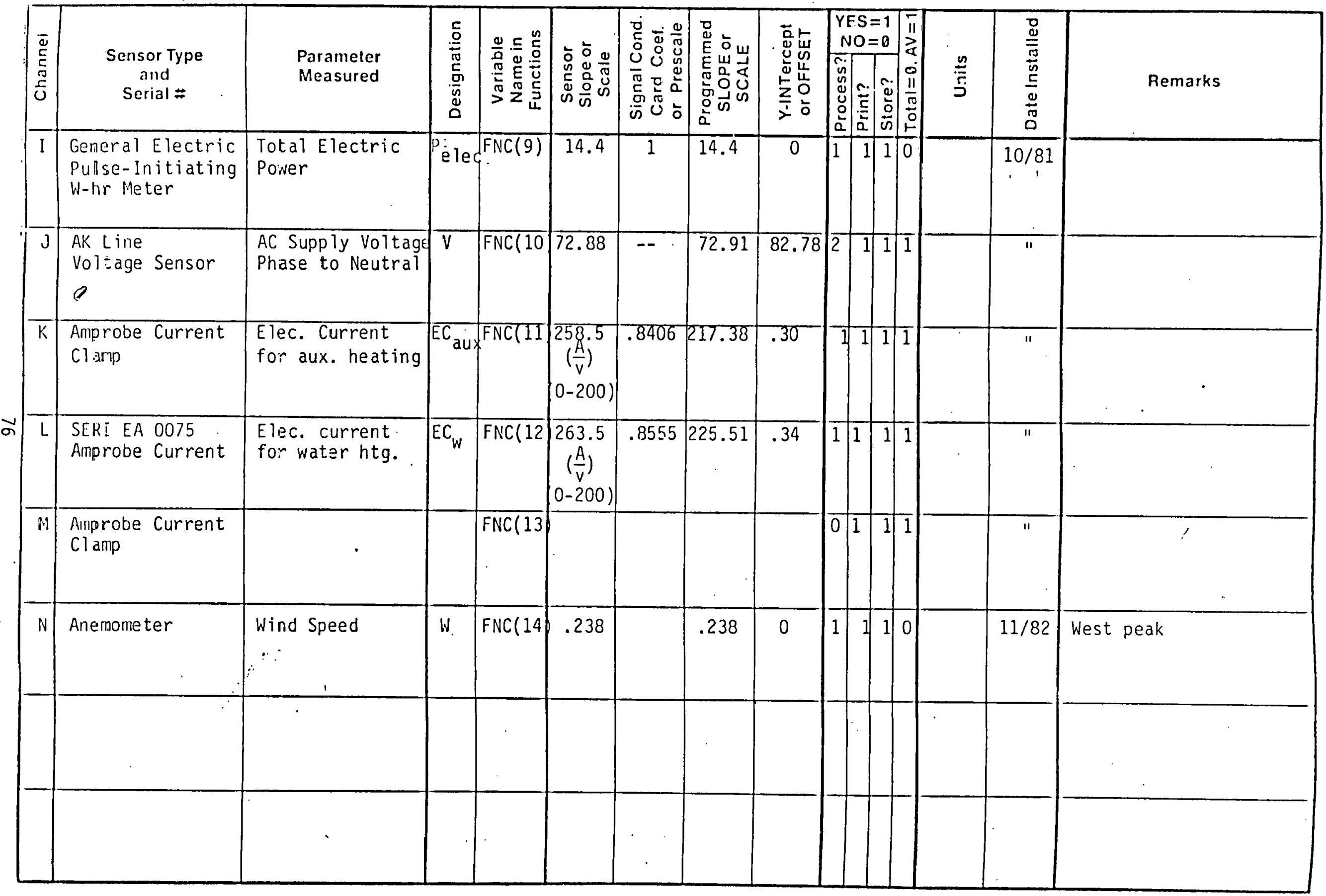




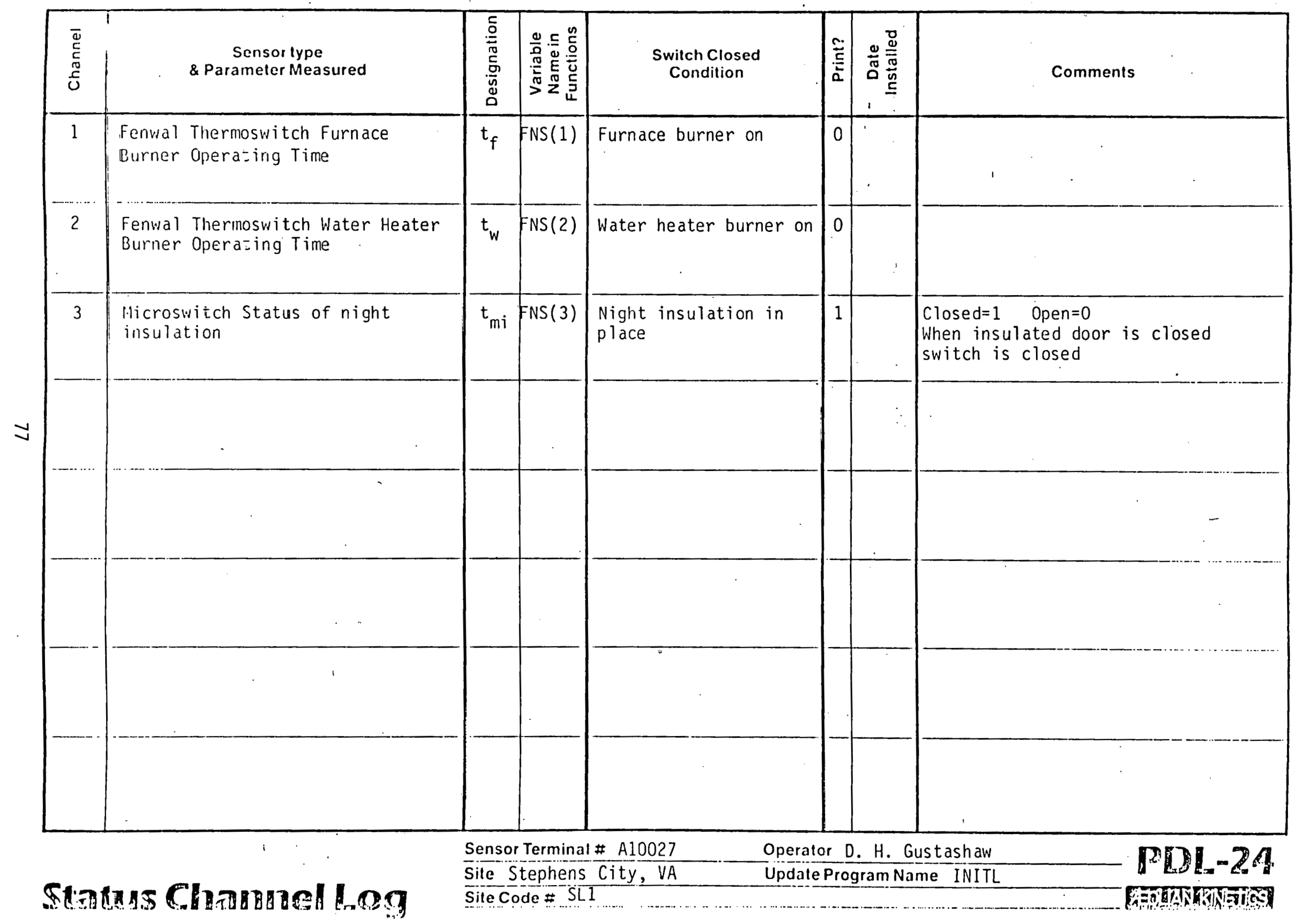


$380 \quad F: Z=: ;=F(50+I)$ $-(C)=1+5+450 \% \mathrm{I}=1 \mathrm{iA}$ LIST

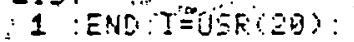

i.10 EIHUT:193,X64:C

$1=1 \Xi-2: Y 1=1$

j. 15 FUF! $=255 T O 15 T E P-$

4:FOKEADOSL, I :NEYYT

20 CT: 1$)=449: 0 T(2)=$

1. $0: 0 T(3)=75 \quad$.

430 FORI $=44 T 045 \cdot F C$

OT $(5)=0 \quad$ OT $(7)=0$ OT $(3)=30$
40 OT

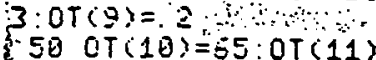

EXT $\because A B$

440 IFFPID $(3)$ OOT (10)

THENF $(4))=F(47)+C O T C$

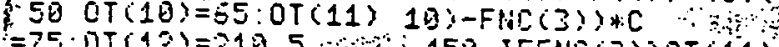
60 OT $(13)=97: 0 T(14$ THENF IFFNC(3) $50 T$ (11)

$2=2:$ OT $(15)=2$ O

iO OT $(16)=0$ OT $(17)=4$ AEB IFF $=3$ THEN5BO

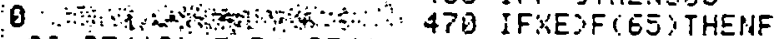

SE OT $(18)=5$. $34: 0 T(1 \quad(E S)=K E: F(E))=0: F(6)$

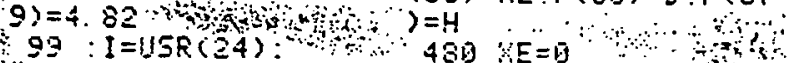

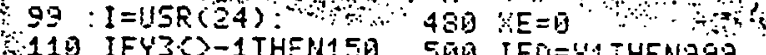

120 FORI=1TO1E:F(I) 519 IFU1=E1THEVII $=0$

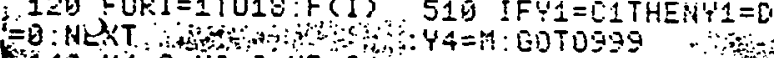

整 $140 \times 4=0: 42=0: 43=0 \quad: 520 \quad 41=0: 45=45+42$

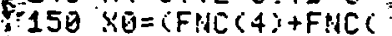

$5)+F R C(5)), 3$

530 FORI $=25 T 031: F(1$

)$=F(I)+F(I-21): N E ! T$ T

$151 \quad K 1=F N C(4): K 2=F M$

$540 F(19)=F(19)+F(1)$

F $)=F(21)=F(21)+F(2): F$

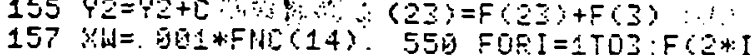

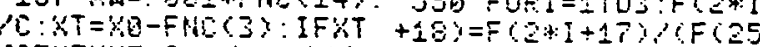

COTHEMKT=O

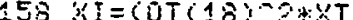

$Y+[1 ;: M E X T$

+ COT(19)

$159 \mathrm{KL}=0 \mathrm{~T}(1) *(1-\mathrm{C} 1-$

OT(12) HENSCO-(1-OT

(13)) 4 (4) $(4)$ )

163 IFXT) OHENF $(4)=$

21) $x+2) / 5 \cdot 15 \% 7$

$578 F(46)=\{F(45) *(4)$

$5-192+84) \cdot 45$

$F(4)+C X L+S I) * x^{\prime} T * C$

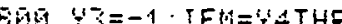
1099

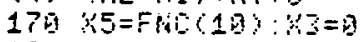

180 FOR! $=1$ TOS KCI $=$

$-3.413+(F)(18+1) 25$

$x+0 T(13+1)+F A(2)+I)$

*:5

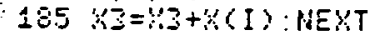

$190 F(E)=F(E)+\cdots(1)+$

OT(5)*DT 4 )*FHSC1)*C $195 \quad x 6=8(2)+10$ T $67 \%+0$

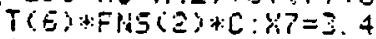

$13+F$ NDC 9 -

$200 F(7)=F(7)+X E: F($

$8)=F(\Omega)+x 7: F(9)=F(3)$

$+\%(2) \quad \cdots \quad$ H

$245 F(5)=F(6)+F(7)+$

$F(C)+F(9)$

250 F (2) $=0 T(2) * F(E)$

2 IF ITTOTHERF $C$ S $=$

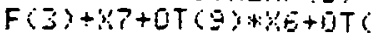

6) $* 10$

ZOD $F(1)=F(4)-F(3)-$

$F(2)$

ZES $Y E=Y E+F N C$ C

(3) $F(1)=C F(11) ; *(T)$

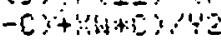

S1: FOEI=1STOMT:FOI CHARHEL OEFAULTE

$\lambda=(F) I)+(Y)-E)+F P Q C I$

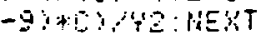

$20=63)=F(13)+C^{\prime}$

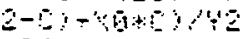

$300=07=: T 00$

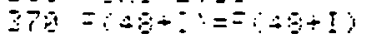

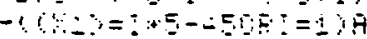

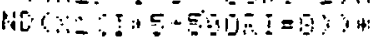

Print THE

OHTH" "A "FEITTDUT"

BQT FUE $=1$ TOET FFI

NT! "F"I;FCI :FCI $=\bar{G}$

NEET

$530 \quad 44=11: 15=15$

999 I $=155023: E N O$

$399 \quad 1=155020 ;:$ ENO

$\therefore$ C $\mathrm{PCLL}-24$

FFOis 1 -

TIME OETIULTS

YEAR $\$ 2$ ?

FFINT INTEFUFL

HES 24 WIVE 6

CH ETUFE INTEEUUAL

HFE 1 MIMS ?

FUTU STUFE IRTEFUAL

FHE HIME

FUWL ZEEO INTEFUAL

HES MIME

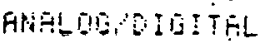

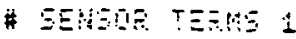

[น⿻

400

$T, \frac{1}{10}$
COE FROCESE 1

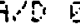

$\because \because 90$

STLFE 1

SLOFE . SZ3. 84

Y-IHT -.37

$82 / 83$ HEATING SEASON

CEE FROEESE 1

H.'C B

$T \therefore 1$

ETURE 1

FRT 1

SLŨfE

Y-INT

652.85

coO

A. CO

T'P 1

STERE 1

PRT 1

SLOFE TES. DS

Y-INT -4.8. 04

COE FROCESE 1

H.'L $\bar{C}$

$T, A 1$

STORE 1

FFT 1

SLOFE

'T-INT

6.928

COF FEOCESS 1

A.S 0

$T \cdot P \perp$

STOEE 1

FFT 1

SLOFE T72.22

'T-IVT -450.

COA FROEESE 1

His 0

$T$ 'A 1

STOES 1

PET 1

SLOFE 653.45

Y-IVT - 455.61

COH FFOCESE

CQIA FETEESE

COR FROCESE 1

Fi. 1

$T \because \bar{A}$ E

STOEE 1

FFiT 1

OCHE 220

UPDATE?

STATUS

SDI FET D

50. FRT

SIS ERT 1

504 FET

505 FET Q

SUE FET O

SGT FET G

SEE PRT 0

UFDATE?

OUTPUT

ETOE:

Fo:

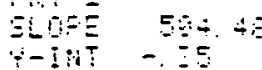

COI FROLESS 1

A.C 1

$T, A$ E

STUEE 1

FFT 1

SCHLE 14.4

COJ FEQRESE 1

H.

$T A 1$

FET 1

SLOFE T2.9日9

'T-IMT $8 \mathrm{ST}$

CBK FFOCES 1

F. $\mathrm{C}$ 日

$T, \overline{1} 1$

STOES I

FET 1

$50 F \quad 215.30$
$Y-I M T$

\# OUTFUT TEEHS

UPCATE? 0

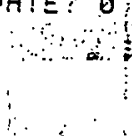

STOEE 1

Co! FFon:

Hid

TH 1

UTE 1

Fi

3050.5 
FURTION OEFHGLTE

\# F)

$F$ I TOEE D FFT 1

$F$ ETRE Q FET 1

$F$ ETOE

$F+$ TOFE G FET 1

F 5 TOE F FRT 1

FE EDE G FET 1

F 7 TUEE 0 FET 1

$F$ ETORE W FFT 1

$F$ STGE 1 FET 1

F1日 STLE O FFT 1

FU1 ETURE Q FRT 1

FI2 ETURE G

FIS TURE Q

Fi4 ETUEE

F15 ETURE G

F1E STORE Q

Fi? STOFE $B$

F13 STITE $B$

F19 ETOFE 0

FQG STOAE D

FQ1 STOFE 0

F2E STOEE 0

FQS STGFE 0

FES STOE O

FS STOFE 0

FEE ETUEE G

F2T STORE

F29 ETUES 0

FEO ETOE 6

FED GTER

FS1 STORE

FZ2 GTORE 0

FZS ETORE B

F3 GTOCE 0

FSE STORE

F36 STORE

F37 STORE G

FSO STORE O

F39 5TOFE 0

F40 STUFE 0 .

F41 STOFE $Q$

F42 STOFE $B$

F43 STUFE O

F44 STOFE 0

F45 STRE 0

FHE STOKE U

F4T STURE O

F48 STURE O

F49 ETORE O

F5G STORE B

F51 STIOEE D

F52 STOAE

FS STUFE

F54 STORE O...

F5S STORE 0

FRT 1

FRT 1

FET 1

FRT 1

FRT 1

FFT 1

FFT 1

FET 1 .

FPT 1

FET 1

FFT 1

FRT 1

FET 1

FFT 1

FERT 1

FET 1

FET 1

FET 1

FET 1

FPT 1

FET 1

FFT 1 i

FET 1

FRT 1

FET 1

FET 1

FRT 1

FRT 1

FET 1

FPT 1

FRT 1

FRT 1

FRT 1

FPT 1

Fit 1

FET 1 ?

FRT 1

FRT 1

FRT 1

FRT 1

PRT 1

FRT 1

FSS STOFE

FRT 1.

FST ETURE $B$

FRT 1

FRT 1

FS\& STORE 0

F59 STURE

F60 STLRE B

FRT 1

FET 1

FRT 1

FRT 1

FS1 ETSFE

FE2 STCIFE 0

FET 1

FRT 1

FES STGRE O FRT 1

FEA ETUEE G

F55 STOFE 0

FRT 1

FRT 1

DQT 1

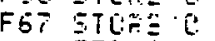

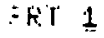


CDE PSOESE 1

523.84

10 OIMUT $(19)$
$1=-23: 11=1$ 15 FOF: $=\angle 55 T O 1$ STEF

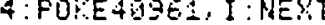
28 OT $(1)=178: 0 T(2)=$ $\because 1$ a OT $(3)=75$

30 OT $(4)=0: 0 T(5)=0$

OT $(6)=0 \quad \because \cdots, \cdots$

40 OT $(7)=0: 0 T(5)=30$

3:OT $(9)=2$ 2

50 DT $(10)=55: 0 T(11)$

$=75:$ OT $(12)=21$ B. 5

60 UT $(13)=.97:$ OT (14

$\gamma=2: 0 T(15)=2$

70 OT (16) = O OT (17)

$B$

80 OT $(18)=5.34: 0 T(1$

$9)=4.82, \cdots, \ldots$

$99 \cdot I=1158(24)$

11B IFY $O S-1$ THEN15B

12B FORI =1TO1S:F(I)

$=0:$ NEXTT

$149 \quad: \angle 4=\overline{0}: \because 2=B: Y Z=\overline{9}$

$15 B: \therefore \bar{B}=$, FHE $(4)+F H C$

5)+FH(C) $) 3$

$151: 1=F H C(4): K 2=F N$

C(7)

$155 \quad 2=\div 2+0$

$157 \quad \because W=$ D.14 $+F H C(14)$

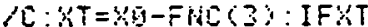
CETHEHTT=E

$156 \because I=C O T(18) \cap 2 \times X T$ +COT 13)*

$159 \quad \therefore L=0 T(1) * 1-61-$ OT I2)

(13):*FHS(4)?

150 IF:TSOTHEVF $C 4)=$

$F(4)+C L L+X i)+X T * C$

$170 \quad \because 5=F M(10): X=0$ 180 FOFI I $=1$ TUE: $:(I)=$ $-3.413 *(F H C(10+I) \%$ )$+0 T(1 Z+I)+F H C(10+I)$ *

$185 \quad \because 3=R 3+3(I):$ NEXT

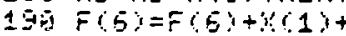

OT (5)*0T (4):FNEC1)*C

$195 \therefore E=B(2)+0 T(7)+10$

$T(6) * F H E(Z)+C: K 7=3.4$

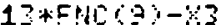

$200 F(7)=F(7)+K E: F i$

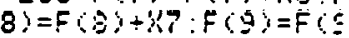

$+\because 3(3)$

$24 B) F(5)=F(E)+F(T)$

$F(3)+F(B)$

$250=2)=0 T(2) * F(t$

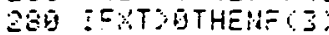

$F(2)+37+0 T C E)+6 E+1) 7$

$8): 0$

ZU日 $F(1)=F(4)-F(3)$

$F(2)$

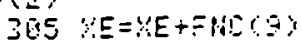

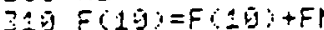

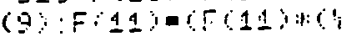

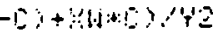

SE FOEI = TOMT:F

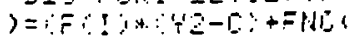

- $\because$ +

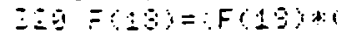

$2-8+6+8$

$\bar{z}=5=$

$=0 \div \div=7 .+1$

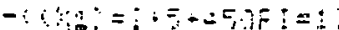

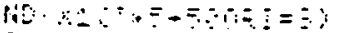

c
FET 1

SLOFE

$Y-I N T$

$-.37$

$(x)=I * 5+451) E Y=1$ i

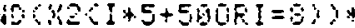

उEB NEXT

$410 \quad \because 4=34+35 * C$

428 FUFI =4BTDA3:FCI CBC FFOCESE 1

H.

$T$ TA 1

5 TURE: 1

PRT 1

SLIFE

'T-INT

$65 \div 55$

$440^{\circ}$ IFFHCO $300 T(10)$

THENF $(4 \vec{i})=F(47)+(1) T($

15:-FNC(Z):

450 IFFNLY3) 30 T (11:

THENF $(4 B)=F(4 B)+C F N E$

(5)-OT (11):

$46 B$ IFF=BTHENSBD

470 IFYESFCES THENF

$(55)=\because, E: F(E E)=[: F(67)$

$\mathrm{Y}=\mathrm{H}$

$48 \bar{B} \times E=$

$5 \overline{6}$ IF D=YATHENS99

$51 B$ IF' $1=\angle 1$ THENY1=0

. $44=11:$ :ETTOS95

$520 \quad 11=0: 35=45+12$

59 FOIFI $=25 T 0 \Omega 1: F C$

\rangle$=F(I)+F(I-21): H E: T T$

$540 F(13)=F(19)+F(1$

)$\cdot F(21)=F(2 A)+F(2): F$

$(Z Z)=F(Z Z)+F(3)$

$550=0 F I=1 T 02: F C 2 \% I$

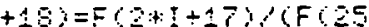

$\because+\mathrm{L} 1 ; \mathrm{NEST}$

56 FUEI = 2TOZS:FOI

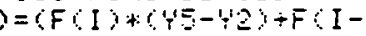

$21)+4: 2$ YS : UE

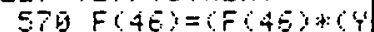

$5-127+\therefore 47+75$

600 $\because 5=-1:$ IF 110

E1D FFIMT!THEC20:"!

GUTH" "Y "FFIHTDLT"

BZO FOFI=

NT! "F"I; F(I):F(i) $)=\overline{0}$ NEXT

$836 \% 4=14: \div: 5=13$

SE I=USF:

29] I=USEC:O: ENO

$\therefore: 001-24$

MIDI TDF:IHG $545 T E A$

TEAR: $\mathrm{BZ}$

FFINT INTEFUAL

HES 24 MINS 6 ?

CH STSFE IHTEEUTL

HFE 1 MIHS D ?

FU!AIE ETOFE IHTERUAL

HFE 9 MIHS BS?

FUNA ZESD IHTEFUAL

HE: 5 मे MIHS मे?

CHARAEL DEFHLILTE

HRFEOTOCIBITFL

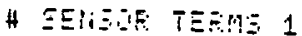

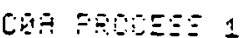

A. $[$ :

1: 0

$\Xi T: 25$

FF: 1

5
$4-195$

COO PROLESS 1

A.' $\left[\begin{array}{c}1 \\ \text { i }\end{array}\right.$

$T A 1$

STOFE 1

FERT 1

SLOPE

768.62

$Y-I N T \quad-458.04$

COE FFOIESE 1

H. $O A$

TA 1

STURE 1

FRT 1

SLOPE 652.95

$Y$ IHT -455.42

CUF FROEESE 1

Tid

TA 1

STOEE:

FFT 1

SLOFE TTZ2

$Y$ IHT -4B日. 28

CDE FFOOESEI

Pis 5

Tis 1

STOEE 1

FF:T 1

SLOE

6.5 .45

$Y$ IUT -45E. 11

COH' FEUCESE

CQI FROEESE 1

$A, O 1$

$T \because A$ D

STUEE 1

FRT 1

SCHEET 14.4

COJ PROCES: 1

4.05

TY 1

STIRE 1

FFT 1

SLIFE T. 9日9

$Y$ YINT 82.279

CBr FEDESE 1

H.O

$T / 1$

ETIEE 1

EFT I

ELPE $\quad 917.3$

$i=11 \quad-45$

C: - Fon: $=1$

Fis

$T / 3$

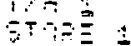

efi.

S:FE

35 ㄱ.

$T, \bar{C}$

STORE 1

$1 / 29 / 83-5 / 20 / 83$

$82 / 83$ HEATING SEASON

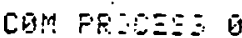

CQN FEDESS: 1

A. 01

$T \cdot \bar{A} \mathrm{~B}$

STOFE 1

FST 1

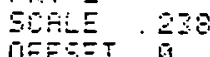

UFCATE:

STFTUS

501 FET

SGE PET

Sig FET

$50 \div \div$ F

505 Fit

$505=5$

5QT FR

509 FET

UFDSTE

OUTFUT

\# OUTFUT TEEHE

UFGATE? 


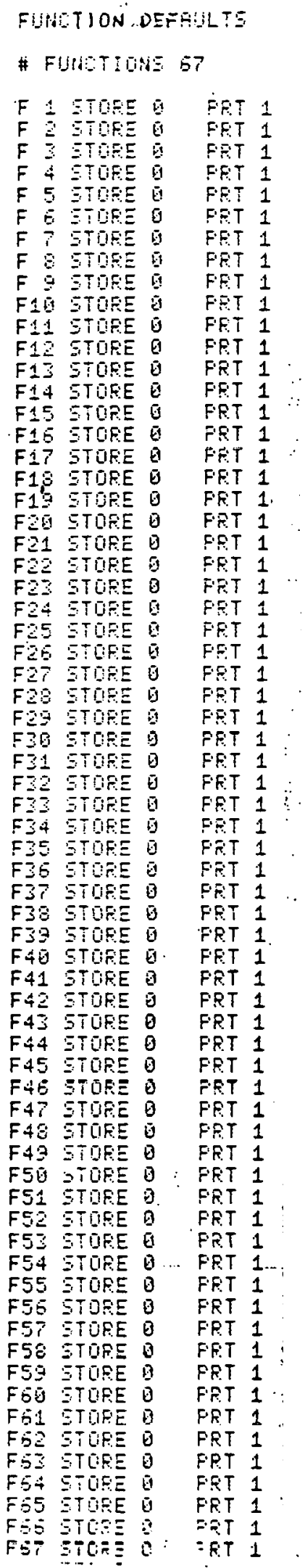




\author{
SITE SN \\ Mr. \& Mrs. Carl Mullin \\ 10649 West Providence Road \\ Richmond, VA 23236 \\ INIT2 Software Installed: 11/19/82
}




\section{SITZ SUMARY SHEET}

Site Identification Number SN]

$\frac{\mathrm{SNI}}{.}$

Buildigi:

Type of Buildiag

General Type of Construction

Ground Coupling
Passive/Hybrid syscea(s):
Dominant Heating Systam Type
Dominant Heating System Area
Other System Type
Other Systeg troa
Nighe insulation

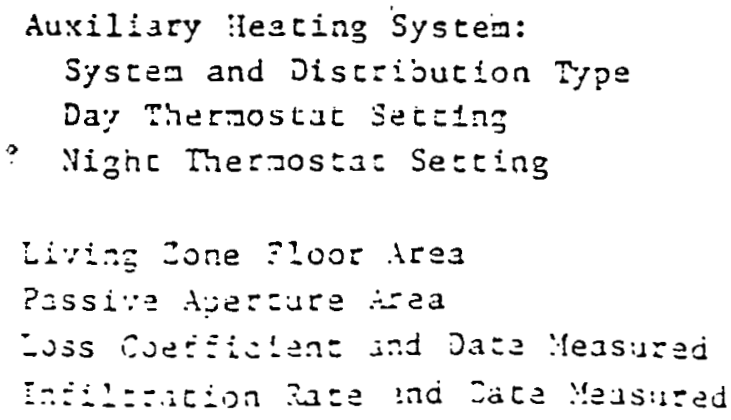

Inst. Contr. Naze ESG, Inc.

Address 2231 Perimeter Park Suite 11 Atlanta, GA 30341

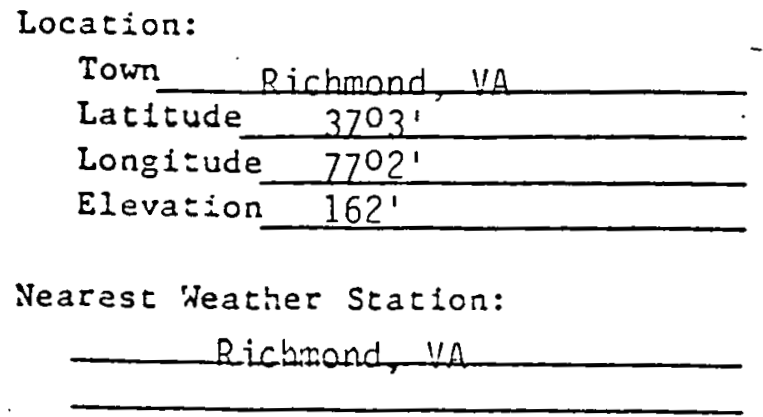

Iwo story residence Wood frame crawlspace

Water walls $260.8 \mathrm{ft}^{2}$ $\mathrm{N} / \mathrm{A}$

$\mathrm{N} / \mathrm{A}$ None

Resistance haseboard heaters 65 OF

$65^{\circ} \mathrm{F}$

$1236 \mathrm{ft}+2$

$260.3 \mathrm{ft} \mathrm{t}^{2}$

$L=268.9 \mathrm{Btu} / \mathrm{hr}$ of : $1 / 31$ and $2 / 1 / 82$

OES $13 /$ SEC $04 \mathrm{PA}-8 / 11 / 32$ 


\section{LEGEND}

ANEMCMETER

WATT HOUR METER

TEMP SEHSOR

VOLTASE SENSOA

ARIP ClaMP

PDL-2 4

PYRAFOMETER

$\stackrel{\infty}{\infty}$
A

$\Theta$ 0

田

田

9

르

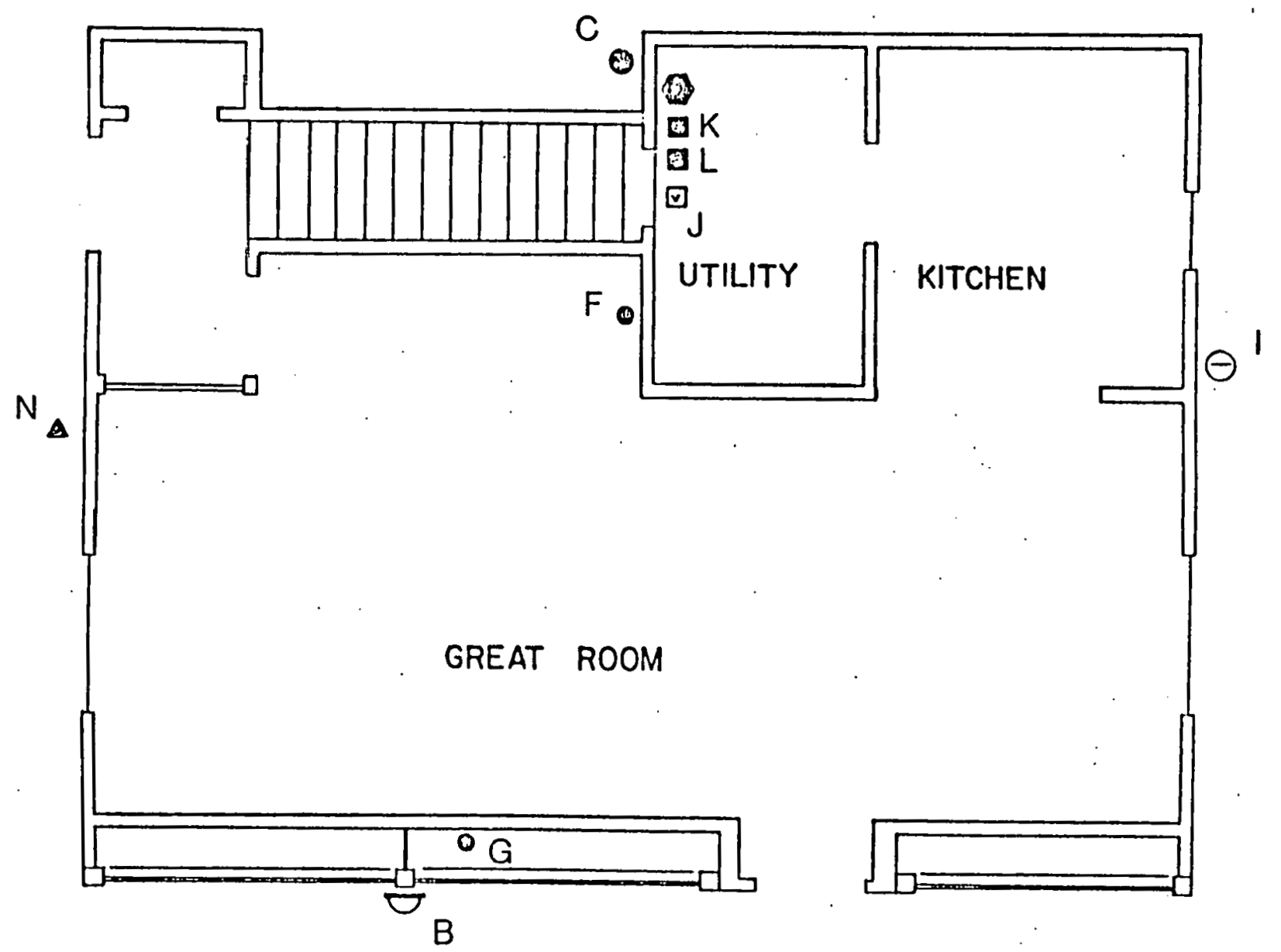

CLASS B MONITORING SITE SN FIRST FLOOR 


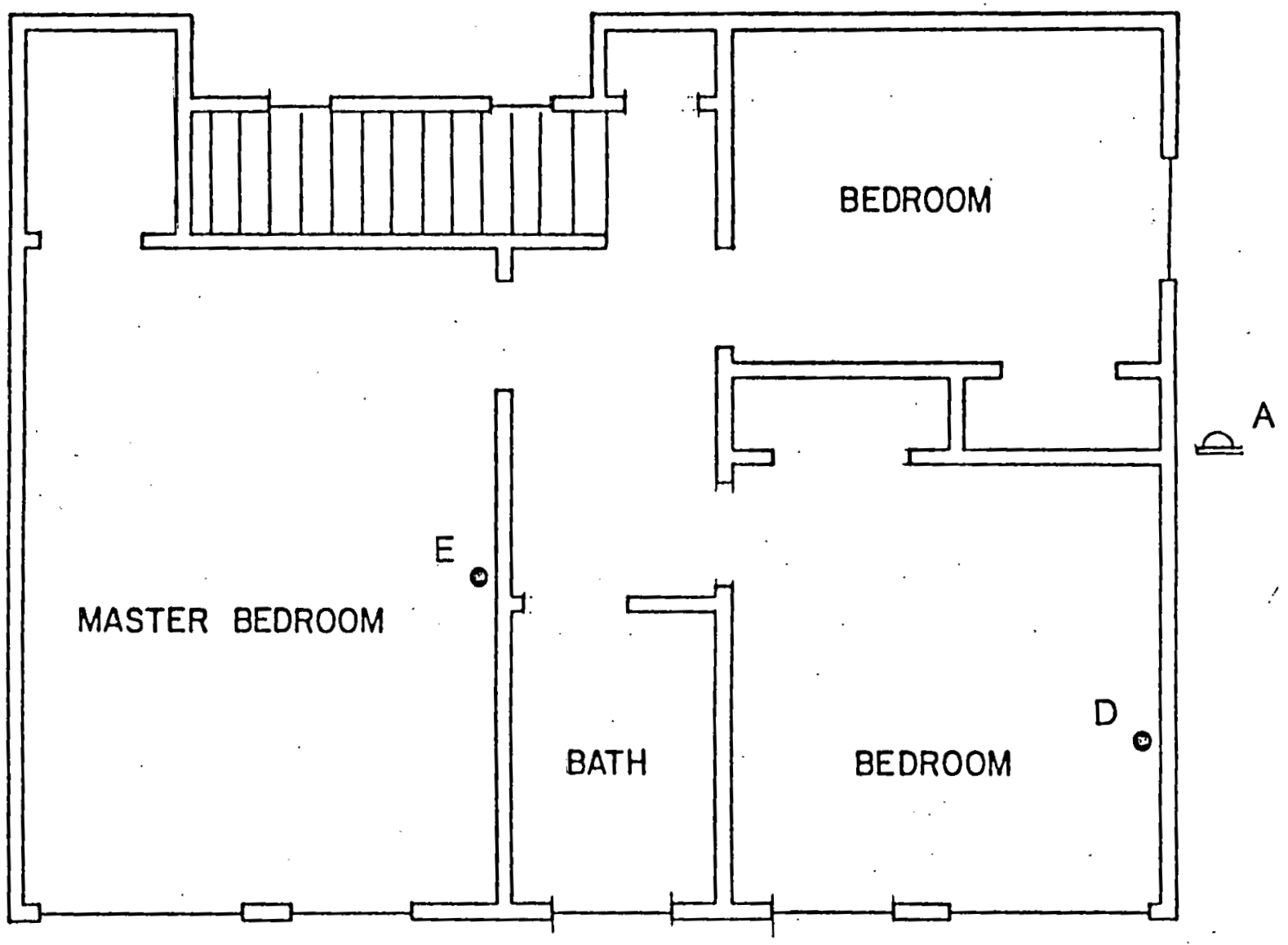

CLASS B MONITORING SITE SN SECOND FLOOR 


\begin{tabular}{|c|c|c|c|c|c|c|}
\hline $\begin{array}{l}\text { Virriabl: } \\
\text { Name in } \\
\text { Functions } \\
\end{array}$ & Description & Designation & $\begin{array}{c}\text { Numerical } \\
\text { Value }\end{array}$ & Units & Line $\#$ & Program \\
\hline $01(1)$ & $\begin{array}{l}\text { Ovcrall heat loss } \\
\text { coefficient }\end{array}$ & L & $\begin{array}{l}441.3 \\
269.0 \\
\end{array}$ & $\frac{B t u h}{O F}$ & & $\begin{array}{l}11 / 19 / 82-1 / 31 / 83 \\
1 / 31 / 81-4 / 30 / 83\end{array}$ \\
\hline$O T(2)$ & $\begin{array}{l}\text { Furnace delivery } \\
\text { efficiency }\end{array}$ & $n_{f}$ & 1.0 & - & & \\
\hline OT $(3)$ & Air infiltration rate & I & .75 & $\mathrm{ACH}$ & & \\
\hline OT (4) & $\begin{array}{l}\text { Furnace burner fuel } \\
\text { flow rate }\end{array}$ & $F_{f}$ & 0 & gal/hi & & \\
\hline OT (5) & $\begin{array}{l}\text { Heat ing value of } \\
\text { furnase fuel }\end{array}$ & $H V F_{f}$ & 0 & Btu/g & 1 & \\
\hline OT $(6)$ & $\begin{array}{l}\text { Water heater burner } \\
\text { f!:el flow rate }\end{array}$ & $\mathrm{F}_{\mathrm{w}}$ & 0 & gal/hi & & \\
\hline $0 T(7)$ & $\begin{array}{l}\text { Heating value of } \\
\text { water heater fuel }\end{array}$ & $\mathrm{HVF}_{\mathrm{W}}$ & 0 & Btu/g & 1 & \\
\hline $0 T(8)$ & $\begin{array}{l}\text { Woter heater jacket } \\
\text { licating factor }\end{array}$ & $Q_{w}$ & 233 & Btuh & & $Q_{W}=(.0833)(40 \mathrm{gal})(140-700 \mathrm{~F})(1)=233$ \\
\hline (0) (9) & $\begin{array}{l}\text { Water heater space } \\
\text { licating factor }\end{array}$ & $N_{w}$ & .1 & - & & \\
\hline OT $(10)$ & $\begin{array}{l}\text { Aux. heating set } \\
\text { point temperature }\end{array}$ & $\mathrm{T}_{\text {seth }}$ & 65 & OF & & \\
\hline OT $(11)$ & $\begin{array}{l}\text { Aux. cooling set } \\
\text { point temperature }\end{array}$ & $\mathrm{T}_{\text {setc }}$ & 75 & $Q_{F}$ & & \\
\hline UT (12) & $\begin{array}{l}\text { Het passive collector } \\
\text { area }\end{array}$ & $A_{C}$ & 261 & $f t^{2}$ & & . \\
\hline $0 T(13)$ & $\begin{array}{l}\text { Hoveable insulation } \\
\text { correction factor }\end{array}$ & $N_{i n i}$ & 1 & - & & \\
\hline OT (14) & $\begin{array}{l}\text { Elec. aux. heating } \\
\text { voltace multiplier }\end{array}$ & $\mathrm{C}_{\text {auxh }}$ & 2 & - & & \\
\hline OT(15!) & $\begin{array}{l}\text { Elec. Water heating } \\
\text { voltage multiplier }\end{array}$ & $c_{w}$ & 2 & - & & \\
\hline OT (16) & $\begin{array}{l}\text { Channel M voltage } \\
\text { multiplier. }\end{array}$ & $c_{m}$ & 0 & - & & \\
\hline OT (17) & $\begin{array}{l}\text { Channel N voltage } \\
\text { multiplier }\end{array}$ & $\overline{c_{n}}$ & 0 & - & & \\
\hline
\end{tabular}




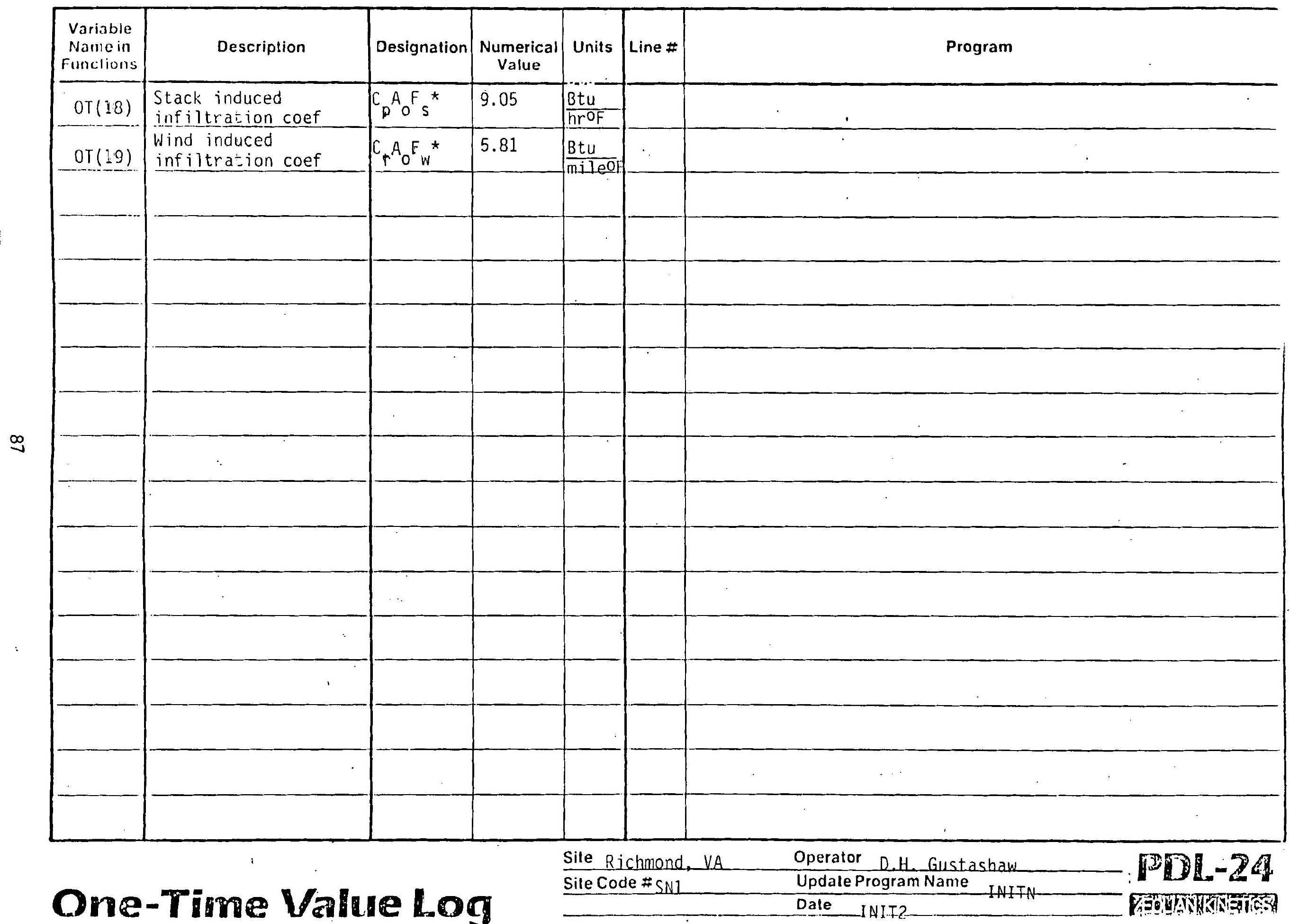




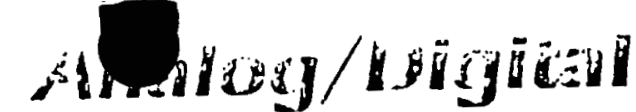 Clazhroballog}

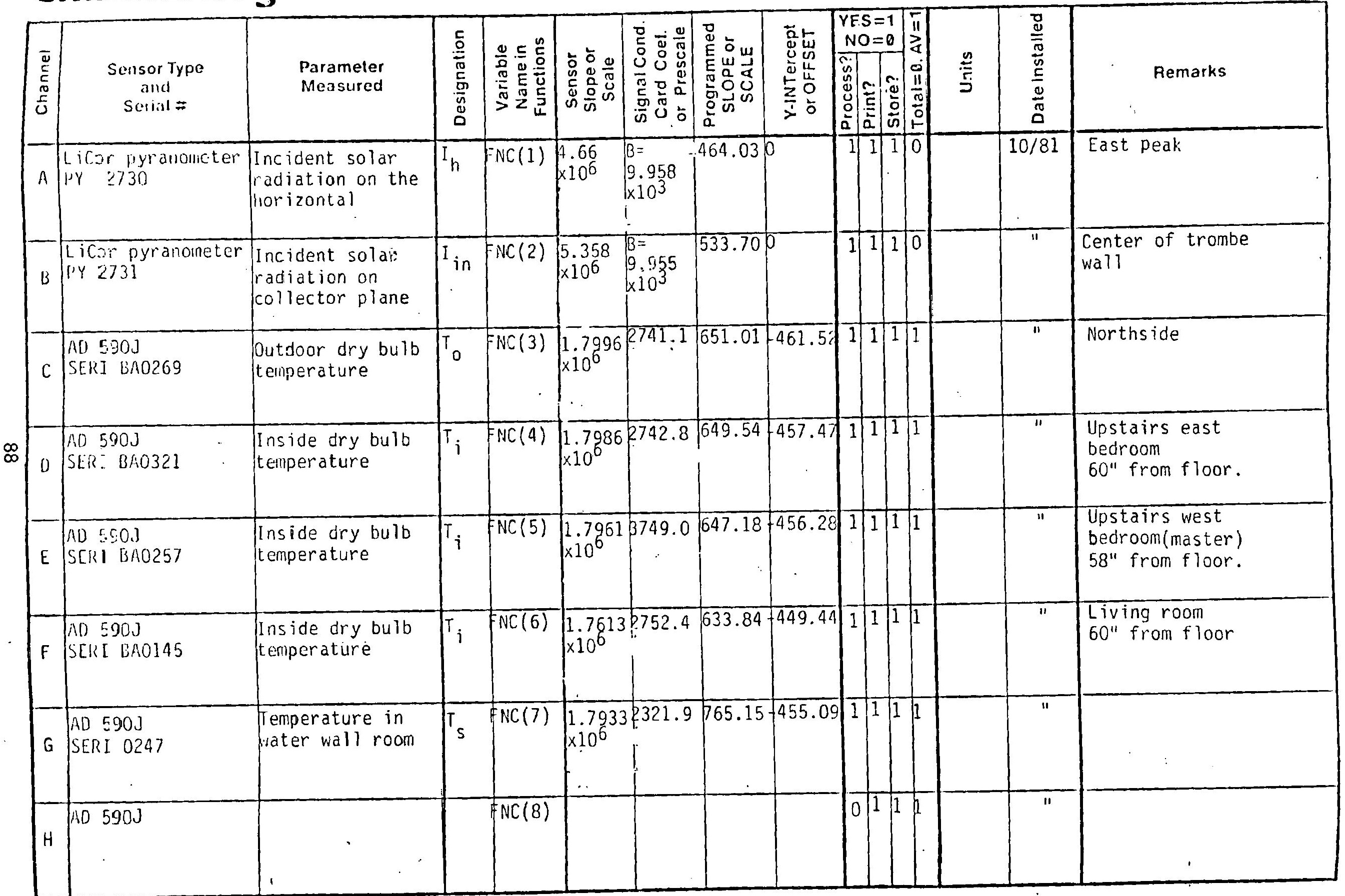




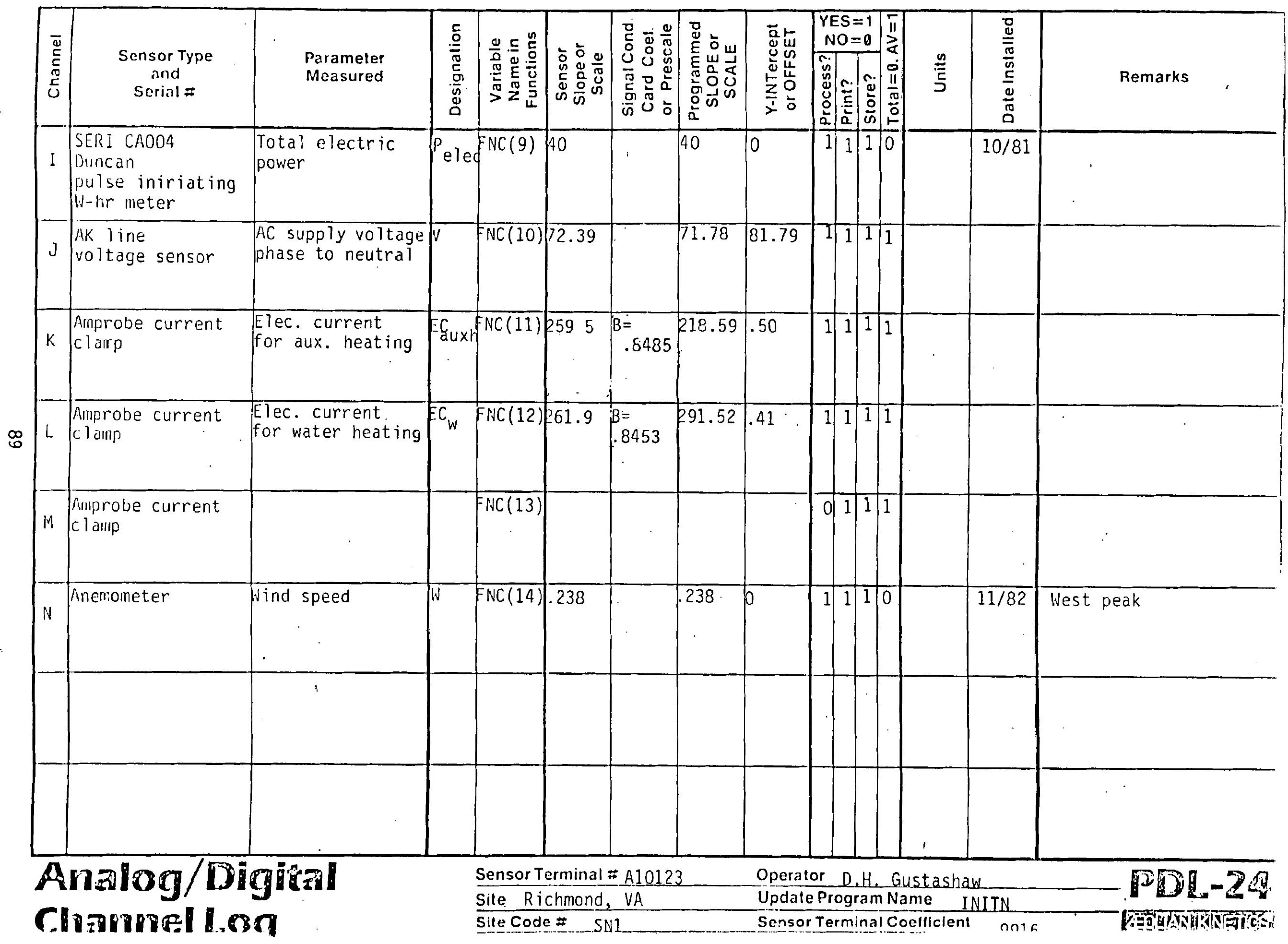




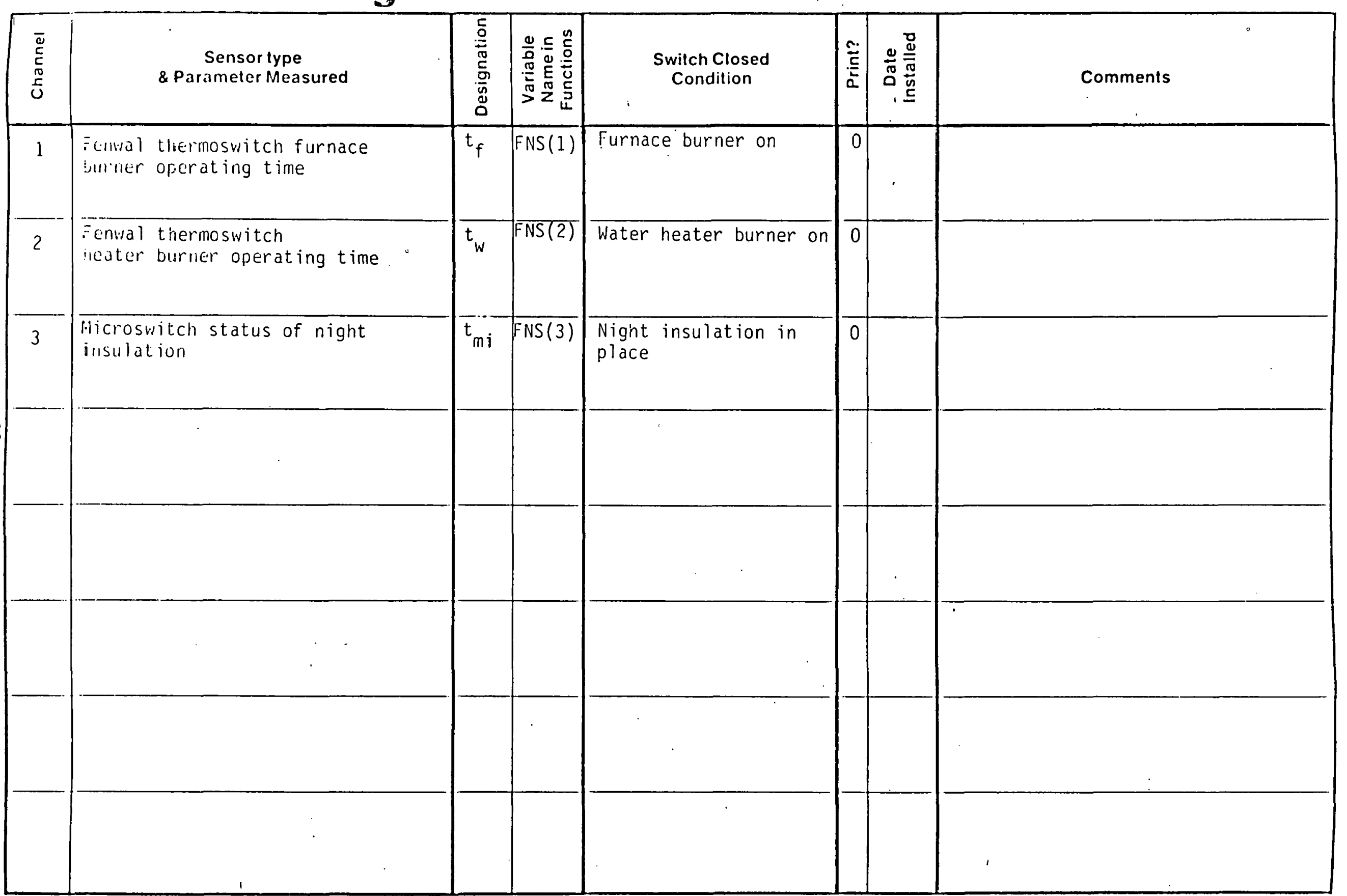


$350=15=:=650+1)$

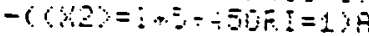
IIST

1 Eno: $:=150(20)$

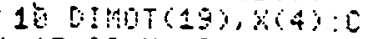
$1=E-: \div 1=1$

15 FOET = $55 T O 1$ STEF-

4:FULETESE: I:NEXT

$\therefore 20$ OTC $1=441.300 T 2$

$\mathrm{p})=1: 0 \mathrm{~T}(3)=.75$

$\therefore 30$ UT $(4)=0: 0 T(5)=0$.

OT $(0)=$

40 OT $(7)=0: 0 T(8)=23$

$3: \operatorname{OT} C:=1$

50 OT (10):=75:0T(11)

$=55: 0 T(12)=261$.

.60 UT $(13)=1.0 \mathrm{DT}(14)=$

2:0T(15)=2 $\therefore$ OT

70 OT $(16)=0$ OT $(17)=$

$0 \ldots$

$=0.01$

90 OT $\{18\}=9.05: 0 T(1$

$99: I=15 P(24)$

110 IF $30-1 T H E$ U150

120 FUFI $=1$ T010:F(I)

$=1: H E K T$

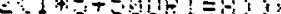

ZOB NEST

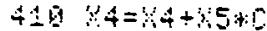

420 FOFI $=40 T 342: F C 1$

$y=F(1)+F ! 13 !-35):$ :

EST

430 FORI $=44 T 045: F C I$

$=F(I)+F M C(I-43): L: N$

E⿰T⿻上丨冖

440 IFFnas

THENF $(47)=F(47)+C O T)$

(1) -FNC (3))

THENF $(48)=F C 48)^{\circ}+C F \mathrm{Ni}$

(3) $-\mathrm{CT}(11)) * \mathrm{C}$........

460 IFF = UTHENSBD

4TB IFYE)F CES)THENF

$(E S)=\because E: F C E \theta)=0, F C E$

$\gamma=\mathrm{H}$

$405 \quad \because E=0$

500 IF $0=1$ THEW

510 !F $1=01 T H E M H=0$

$Y 4=19$ : GOTOSOS

520 Y $4=0: 5=15+12$

- 530 FURI $=25 T 031: F C I$

$y=F(I)+F(I-21): N E X T$

54 G $F(19)=F(19)+F(1$

$\checkmark F(21)=F(21)+F(2): F$

$Q 23)=5(23)+F(3)$

\& 550 FUFI=1T03:F62:I

$+15)=F(2) I+17) \rightarrow(F)$

$\because+E 1\rangle: M E Y T$

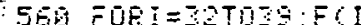

$Y=C F(I)+C Y 5-Y Z)-F C I-$

$21\}+12 \times 15$ MEXT

57 I $F(46)=C F C A B)(C$ $5-2)+34)+5$

800 YS=-1 IFM= ITHE 1993

Q1日 FF:HT!THECOU, “M ONTH"WA "FERTHUT"

Q20 FGEI=4OTDET:FRI NT! "F"I:FCIY:FCD MEST

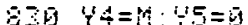

9.9 !=ISRCZ: ENO

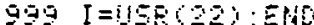

$6900-24$

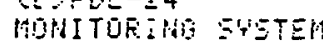

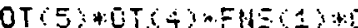

$195 \quad \therefore E=x(Z)+\overline{0} T(7) * 1]$

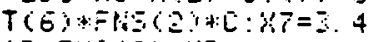

13*FliL $Q 9)-13$

$3205 F(T)=F(T)+R E: F C$

$8)=F(\theta)+K T: F(\bar{g})=F(g)$

$+3(3)$

$240) F(5)=F(E)+F(T)+$

$F(\theta)+F(\theta)$

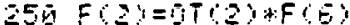

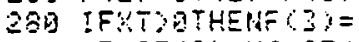

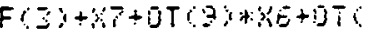

Qidis

SG日 $F(1)=F(4)-F(Z)-$

$F(2)$

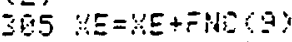

$310=(10)=F(10)+F P$

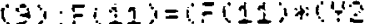

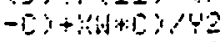

315 FOEI = $2 T 017: F$ I

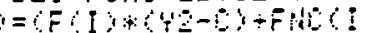

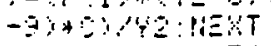

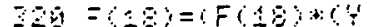

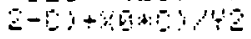

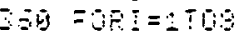

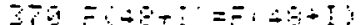

$-4=10-6=0$

15 $x-10=9$

TFAF 82 ?

FFINT INTEEUAL

HRE ZA MINS O ?

FUAO STOFE IRTERUHL

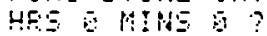

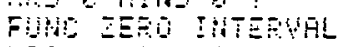

HAE THWO?

CHANAEL DEFALIS

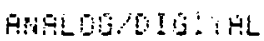

\# EEHEOR TESHE 1

$0850005=$

Ais 6

$T S$

ET:
45 IFFMECSOTC11:

Cos $: \because: 2 \equiv: \sum 1$

म. 6

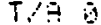

ETSEDI

FRT 1

3 GE

Yे-
53.7

6
INIT $\mathbf{N}$

Cof FFOE:SE

Bid 0

$T, P 1$

STORE 1

FET 1

SLUFE E.51.01

$Y-I N T-451.52$

COD PFULESE 1

मी. 0

$T \because F 1$

STGRE 1

FF?

SLOPS 64954

$Y$ YNT -457.47

$11 / 19 / 82-1 / 31 / 83$

CDE FRDESE 1

में

$T / \hat{H} 1$

ETOES 1

FET 1

SLOEE E.47.48

$\mathrm{i}$-inT -4.5 .28$

COF PEOESES 1

a d 0

T.P 1

STOEDA

FET 1

ZUEE EZS

YIIHT - 443.4

CuE FFOBESE 1

Fi, 0

T. 1

STOPE 1

FET 1

ELFE TES 15

YINT - 455.62

COH FFOEESE

$82 / 83$ HEATING SEASON

[DIf fe.

CDH FFOEES 1

A. 01

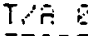

STOEE 1

FreT 2

OCFE

UFDETE? 0

ETPTHS

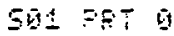

$502=0 \quad 0$

502750

$54=05$

S日5 $50 \div$

S月E PET

$567=07$

$50=5$

UFDA

OUTFUT

\# DUTFIT TEFHE Q

UFEFTE?

CDI FPOLES 1

Fin 1

STOE

FFT 1

SCFIE 40

UFFEET G $\rightarrow$

CH STORE IHTERUAL

HES 1 MILS $5 ?$

FE:

$\begin{array}{lll}3-0 & 4\end{array}$
Caj Frotes: 1

Hio

$T, \bar{A} 1$

STUE: 1

FFI 1

SLDE T2.779

H-INT SI.759

ENA FEDEES 1

F. 6

$T \because A$

ETrOE 1

Fit?

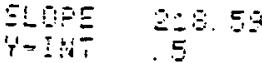

G. FFOEZI

400

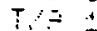

301

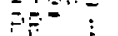

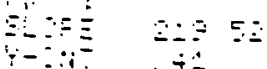


FUHOTION OEFHILTS

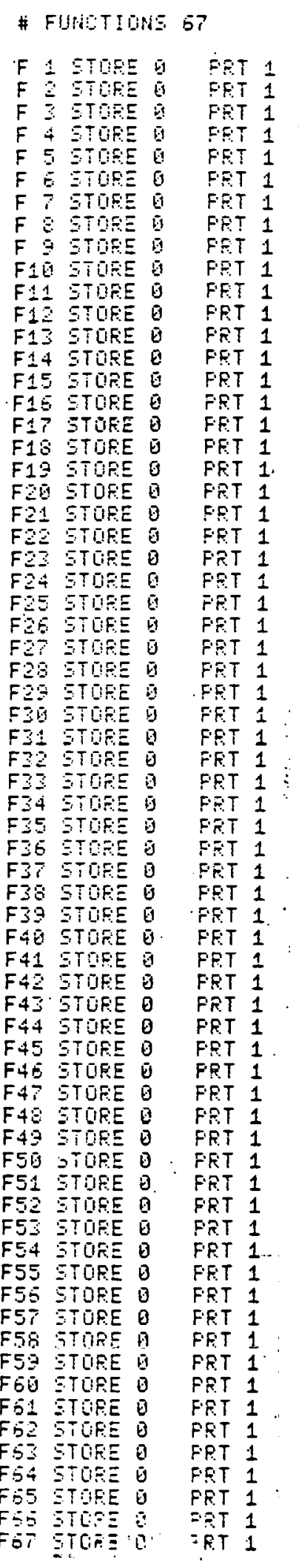




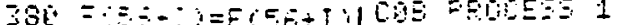

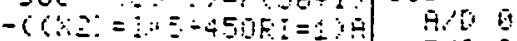

Mrow $1+5+565 I=0 \%$

LIST

10 OIHOT 193, : $14::$ $1=1=-0.14=1$

15 FUFT TSETOISTEF-

4:FOKE 4 OSI, I:PEYXT

20 OT $(1)=259:$ UT $(2)=$

$1: 0 T(3)=.75$

30 OT $(4)=0: 0 T(5)=0$

OT $(6)=0$

40 OT $(7)=0:$ OT $(8):=23$

$3: 0 T(P)=1$

5B OTCIB: $=75:$ OTC11:

$=65:$ OT $(12)=261$

660 OT $(13)=1:$ OT $(14)=$

$2: 0 T(15)=2$

70 OT $(16)=0$ : OT $(17)=$

80 OT 18$)=0.05: 0 T(1$

$9)=5.81$ :

$99: I=U S R(24)$.

$\because 110$ IFY $C D-1$ THENISO

र. 120 FURI $=1$ TU1E:F(I)

$=B:$ NEXT $\rightarrow$ TOA

$140 \quad x 4=0: Y 2=0: Y 3=0$

$15 B \quad X \bar{O}=C F M(4)+F M C$

$5)+F(15(5)) 3$

$151 \quad X 1=F N C(4): \because 2=F$

C(7)

$15513=182+5$

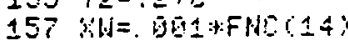

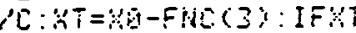

CBTHEVMT $=$

$158 \% I=\operatorname{CoT}(12) \cdots+2$

+ COT(19)*

159 : $2=07(1) * 61-61$

OT 12 in: $N=43-6-01$

(1)

16E SFSTYUTHEHFCA

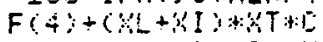

$170 \quad y=70010 ;: 2=$ 180 F $0 \mathrm{I}=1 \div 03 \mathrm{3}(\mathrm{I})$

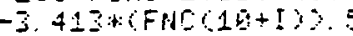

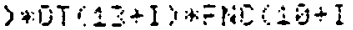

*

$185 x=\times 3+K 6$ I : NEKT

$130=(0)=\bar{F}(6)+3(1)+$

OT $(5)$ OT $(4)$ FNEC1)*

$195 \quad \forall E=8(2)+0 T(7) * 0$

$T(B)+F S(2)+C: X_{T}=3.4$

$13+F \mathrm{AO}(9)-\mathrm{XS}$

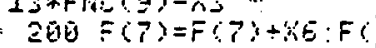

$8)=F: B)+K(F(9)=F(9)$

$+i 63$

$240 F(5)=F(\sigma)+F(T)+$

$F(E)+F(B)$

255 F 2 ) $=1$ (2) $F(E)$

200 IFKT 3 G THEMF

FCSU+YT+DTCOYHE+DT

$8 ;+10$

SBu $=(1)=F(4)-F(3)-$

$F(2)$

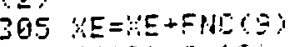

$310 \%(10)=r(18)+F H 0$

(9) $F(11)=C F(11) * C^{\prime}$

$-0+x+10)+2$

315 FOEI=-TO17:FG

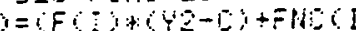

$-900120$

$32=69 \%=618)+6$

$2-6-3000$

$30=0=0$

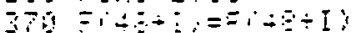

$-62=-0=-5=0=1$

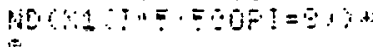

E
उ9耳 :IEXT

$410 \quad 34=\$ 4+15 * 0$

420 FURI $=407043: F(I$

)$=F(I)+F H S C I-35)+H_{L}: M$

EXT

430 FURI $=44 T O 4 S: F C I$

$=F(I)+F N C(I-43) ;: E: N$

ETT

440 IFFNCOZYOT610:

THENF $(47)=F(4 T)+C O T C$

19) -FNE $(3:) * C$

THENF $(4 B)=F(A B)+(F N C$

(3)-OT(11);:C

460 IFF $=$ ITHEHSOB

$(E 5)=\{E: F(E)=0): F(6)$

$j=\mathrm{H}$

$480 \quad 3 \mathrm{E}=0$

510 IF'1 1 ITHEN': $=0$

$44=M \cdot G 0 T 0959$

$520 \quad 41=0: 15=45+12$

$5 S D$ FURI $=25 T D Z 1: F(I$

$=F(I)+F C I-21) \cdot 15 \% T$

546 F $619 ;=F(19 ;+=1$

$\because F(2)=F(21)+F(2): F$

$2 Z)=F(23)+F(Z)$

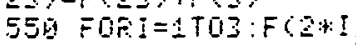

$+1 E)=F(2+I+17)$ C $(2)$

+ + 1$): N E R T$

500 FUF $=2$ TOZS:

$=A F(I)+C H E-T 2)+F(1-$

21) 2 Q

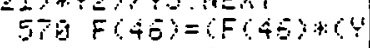

$=-12)+843,15$

Q60 $Y Z=-1: I F H=H 4 T E$ 1949

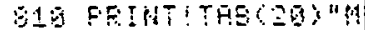

OHTHI"LCFETATOUT"

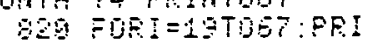

UI! "ELI I $C$ C I :FCI $)=0$

$H=Y T$

820 \% $4=19: 45=0$

$939 \quad I=658023:$ ENO

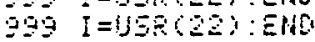

- $604-24$

MHITBRI IM!O EYSTEM

FEOT I $\because \because 3$

TIUE SEFFU IS

HEAF: $B S$ ?

FE INT INTEFVA:

HEE 24 MINE 1

OH STOEE IMTERUAL

HES MING

FUUL STUED IHTEEUPL

HES 9 MINS 9 ?

FUAC EEET IATERUAL

HES 5 MIMS

VHAMUEL DEFGIIT TS

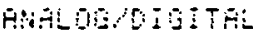

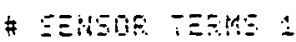

$06=605=2$

$\therefore 0$

$-\therefore 9$

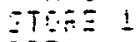

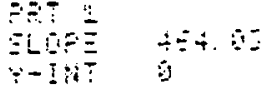

450 IFFNC $3300 T(11$ )

4TE IFNEYSCESTHENF

505 IF $=14$ THENOSO
$T: 4$

STHES 1

FFE: 1

SLBE $5: 72 / 83$ HEATING SEASON

Y'IVIT

CDO PFOEEES1

Fis 6

$T / A 1$

STORE 1

FET 1

SLOFE ES1.81

Y-INT -451.52

CQO FRUEES 1 ........

A. $\mathrm{C} \quad \mathrm{C}$

$T \therefore A$

STOEE 1

FRT 1

SLOPE S4.54

YIVT -45i.4i

COE FROTEES 1

F.' $D$

T.A 1

STUFE 1

FET 1

LLFE $\quad 647.18$

Y-IVT -450.28

LOF FRUESES 1

A.S 0

$T A 1$

STORE 1

FFT 1

SLOFE ESE. 84

YIITT -449.44

CoS FeOEES 1

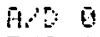

$T, 1$

STOFE 1

FET 1

SLOPE

HIVT - IUE

COH FOOEESE

CO! FROEES 1

H.' 1

$T \wedge$

STIRE 1

FRT 1

SCALE 40

OFFEET अं

COJ FEOSESS 1

A. 0

$T \therefore 1$

STrEe:

FET 1

SLOFE 71.779

Y-IUT 81.93

Dat FEUES 1

A.

$T 1$

STOEE I

FOT 1

QLOE

YIVTT

CoL $=0 \equiv \equiv 1$

ing

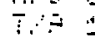

$3=$

$3=$

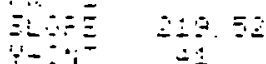

Con Frous

Con PEOEES 1

Hid 1

T保要

STONE 1

FET:

5itE . 230

UFमA:

STHYS

SD: FoT

592 Fe?

SQI Fot

$50=5$

$5 \mathrm{x}^{2}=\mathrm{T}$

506 FoT

$507 \mathrm{FT}$

SGE FET

UFCATE?

OUTFUT

\# OLTFUT TEERE B

UFLATE? B 
FUHITION OEFFILTS

\# FUMUTIONE 57

F 1 ETIPE

FFT 1

$F=0$ TEE

$F$ STOFE

$F+$ TUFE G

$F$ S TOEE

$F E$ EOEE

F 7 STORE

F 8 STDEE

$F 9$ STRE

F40. STORE O

F 1 STORE B

F12 STORE B

FA3 STURE Q

Fit STQFE

F15 ETURE

F15 STOFE O

Fi? STORE E

F13 STORE O

F19 STORE

F20 STORE G

FQ1 STORE O

F2Q STOFE

F23 STLAE $B$

FOA ETOED

FES STREE

FES TRE

FQT STORE

F28 ET0PE

FOS ETOFE

F3G STORE

F1 ETURE

F32 STORE

FEZ STOFE

F34 ETORE

F35 STORE

F36 ETORE B

F37 STURE

F38 STOEE 0

FS马 STUEE

FAO ETOFE

F41 ETORE 9

F42 STURE B

F4T ETUFE

F44 ETUIEE 0

F45 ETURE

F45 STORE O

F4T ETURE

F49 STLRE

F49 STORE 0

F5O STORE O

F51 ETORE 0

F5: ETUEE 0

F5. STOFE

F54 STORE $B$.

F55 STCRE

F5S STORE O

FST ETURE 0

F5E STRE 0

F59 STURE B

FEG GTURE O

FD1 ETERE

FG2 STLEE O

FDE STLRE O

Fo4 ETURE 0

FES STOEE G

FOS ETLE

FBT

FFT 1

FET 1

FFT 1

FET 1

FET 1

FET 1

FRT 1

FET 1

FET 1

FET 1

FRT 1

FRT 1

FET 1

FRT 1

FRT 1

FFT 1

FFT 1

FET 1 .

FRT 1

FRT 1

FRT 1

FRT 1

FET I

FRT 1

FRT 1

FET 1

FET 1

FFT 1

FET 1

FPT 1

FRT 1

FFT 1

FET 1

FET 1

FET 1

PRT 1

FRT 1

FRT 1

FET 1

FRT 1

FRT 1

FRT 1

FRT 1

FRT 1

FRT 1

FET 1

FRT 1

FRT 1

FRT 1

FRT 1

PRT 1

PRT 1

FRT 1 .

FF:T 1

FRT 1

FRT 1

FET 1

FRT 1

FRT 1

FET 1

FRT 1

FPT 1

FRT 1

FRT 1

जET 1

$=\mathrm{Bi} 1$ 


\section{APPENDIX A}

\section{SITE PHOTOGRAPH TABULATION}

A number of photographs were taken to provide installation details of most of the sensors used as part of the instrumentation system. Elevation views of each house were also made as part of the overall documentation and will help provide information on the passive design features. These photographs were in $35 \mathrm{MM}$ slide format and were provided to SERI in transmittals on February 15 and April 14, 1983.

The following table provides a listing of these photographs for each site location. 
Channel

\section{C}

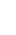

F

政

F

$\begin{array}{ll}\text { G } & \text { Inside Temperature } \\ \text { H } & \text { Inside Temperature } \\ \text { I } & \text { Watt-Hour Meter } \\ \text { J } & \text { Voltage Sensor } \\ \text { K } & \text { Auxiliary Heat Amprobe } \\ \text { L } & \text { Water Heat Amprobe } \\ \text { M } & \text { Air Conditioning Amprobe } \\ \text { N } & \text { Anemometer }\end{array}$

Status 1

Status 2

Status 3

Status 4

Status 5

Status 6

Status 7

Status 8

North Elevation

South Elevation

East Elevation

West Elevation
$\begin{array}{llllllll}S A & S B & S F & S G & S J & S K & S L & S N\end{array}$

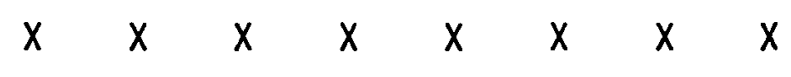

$\begin{array}{llllllllllllllllll}x & x & x & x & x & x\end{array}$

$\begin{array}{llllllllllll}x & x & x & x & x & x\end{array}$

$\begin{array}{lllllll}x & x & x & x & x & x & x\end{array}$

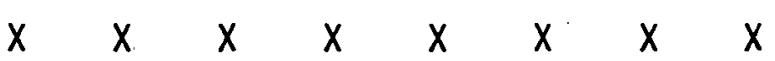

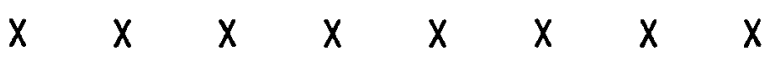

$x \quad-x \quad x \quad x \quad-x$

$\begin{array}{llllllll} & x & x & - & - & - & - & - \\ x & x & x & x & x & x & x & x\end{array}$

$\begin{array}{lllllll}x & x & x & x & x & x & x\end{array}$

$-x-x \quad x \quad x \quad x$

$-\quad-x \quad x \quad x \quad x$

$\begin{array}{llllllll} & x & - & x & & - & - \\ x & x & x & x & x & x & x\end{array}$

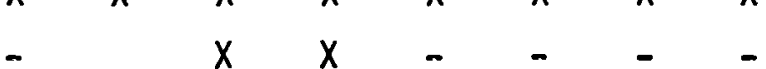

- $x$ x $x-.-$

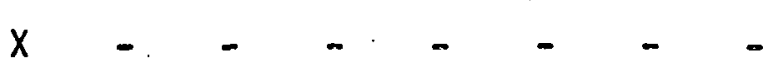

$-x-2-x-0-$

$-x-x-\frac{-}{-}-\infty$

- $x-0-0-0$

- $-\quad-\quad-\quad-\quad-$

$\begin{array}{llllllll}- & x & - & - & - & - & - & - \\ x & x & x & - & -\end{array}$

$\begin{array}{lllllllllllll}x & X & x & x & x & x & x\end{array}$

$\begin{array}{lllllll}x & x & x & x & -x & x & x\end{array}$

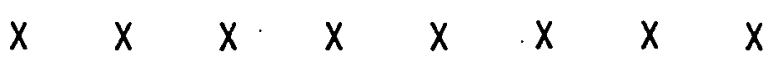

$x \quad x \quad x \quad x \quad x \quad x \quad x$ 